Review article

\title{
A glance over doxorubicin based-nanotherapeutics: From proof-of-concept studies to solutions in the market
}

\author{
Mara Gonçalves ${ }^{\mathrm{a}}$, Serge Mignani ${ }^{\mathrm{a}, \mathrm{b}}$, João Rodrigues ${ }^{\mathrm{a}, \mathrm{c}}$, Helena Tomás ${ }^{\mathrm{a}, *}$ \\ ${ }^{a}$ CQM-Centro de Química da Madeira, MMRG, Universidade da Madeira, Campus Universitário da Penteada, 9020-105 Funchal, Portugal \\ ${ }^{\mathrm{b}}$ Université Paris Descartes, PRES Sorbonne Paris Cité, CNRS UMR 860, Laboratoire de Chimie et de Biochimie Pharmacologiques et Toxicologique, 45, rue des Saints \\ Peres, 75006 Paris, France \\ ${ }^{\mathrm{c}}$ School of Materials Science and Engineering, Center for Nano Energy Materials, Northwestern Polytechnical University, Xi'an 710072, China
}

A R T I C L E I N F O

\section{Keywords:}

Cancer

Chemotherapy

Doxorubicin

Nanotherapeutics

Delivery systems

Clinical trials

\begin{abstract}
A B S T R A C T
Cancer is one of the leading causes of death worldwide and, as such, efforts are being done to find new chemotherapeutic drugs or, alternatively, novel approaches for the delivery of old ones. In this scope, when used as vehicles for drugs, nanomaterials may potentially maximize the efficacy of the treatment and reduce its side effects, for example by a change in drug's pharmacokinetics, cell targeting and/or specific stimuli-responsiveness. This is the case of doxorubicin (DOX) that presents a broad spectrum of activity and is one of the most widely used chemotherapeutic drugs as first-line treatment. Indeed, DOX is a very interesting example of a drug for which several nanosized delivery systems have been developed over the years. While it is true that some of these systems are already in the market, it is also true that research on this subject remains very active and that there is a continuing search for new solutions. In this sense, this review takes the example of doxorubicin, not so much with the focus on the drug itself, but rather as a case study around which very diverse and imaginative nanotechnology approaches have emerged.
\end{abstract}

\section{Introduction}

Cancer is characterized by the uncontrolled growth and spread of abnormal cells, being a very important human health problem. In 2018 and according to estimates of the World Health Organization, there were about 18 million new cancer cases and 9.6 million deaths occurred as a consequence of cancer [1]. Although the genesis of cancer is related with an accumulation of mutations in critical genes that control cell behaviour, it is well known that factors like increased population ageing, environmental problems and adopted lifestyle, may strongly contribute for its incidence [2]. In this scope, it is of extreme importance to develop new strategies for cancer treatment that are more effective and prevent unnecessary deaths.

Nanomaterials, that may be defined as materials that have at least one dimension at the nanoscale [3], can provide solutions for the wellknown problems associated with conventional anticancer therapy [4]. Particularly, they can be used as drug delivery vehicles and thus help to increase the effectiveness of medicines and simultaneously reduce their negative side effects. Indeed, nanomaterials are interesting tools for the delivery of therapeutic agents since they possess the right size to circulate inside the human body and to interact with biological targets, like macromolecules, cells and cell organelles [5,6]. In addition, depending on their size, shape/architecture, chemical composition and surface functionalization, nanomaterials may exhibit special chemical and physical properties that will impact their biological behaviour $[7,8]$. Regarding cancer treatment, their use as drug carriers may have a direct impact on the pharmacokinetics (PK) of the drug, including on the extent of drug's cell uptake, and as such on its efficacy [7,9]. In the case of solid tumours, it is also believed that nanomaterials may accumulate in the tumour site through the "Enhanced Permeation and Retention" (EPR) effect $[9,10]$ which will be explained later in this review. Nanomaterials can also be designed to specifically target cancer cells (targeted nanomaterials) [11-19] or to release the drug only under the presence of specific environmental stimuli [20-29], thus helping to diminish unwanted side effects. Other advantages associated with the use of nanomaterials as drug delivery platforms include: the sustained delivery of the drug [17,30-33]; the delivery of more than one drug at the same place and at the same time (co-delivery of drugs) $[15,23,25,28,34-40]$; the possibility of transporting poorly soluble drugs that otherwise could not be administered by simple intravenous injection [41-43]; the protection of the drug from possible existent damaging environmental conditions [44-49]; the potential of bypassing

\footnotetext{
* Corresponding author.

E-mail address: lenat@uma.pt (H. Tomás).
} 
<smiles>COc1cccc2c1C(=O)c1c(O)c3c(c(O)c1C2=O)CC(O)(C(=O)CO)CC3O</smiles>

Adriamycinone<smiles>CC1OC(O)CC(N)C1O</smiles>

Daunosamine

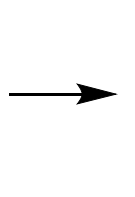

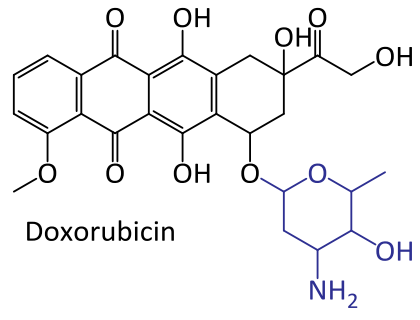

Fig. 1. Chemical structure of DOX. DOX molecule is based on a water-insoluble aglycone (adriamycinone, with lipophilic character) and a water-soluble amino-sugar moiety (daunosamine, with hydrophilic character); the adriamycinone consists in a tetracyclic ring with a quinine-hydroquinone group nearby; the amino-sugar moiety is linked to one of the rings through a glycosidic bond [63]. drug resistance mechanisms [50-52]; and the chance to associate drugs and contrast agents for medical imaging techniques in the same nanodevice thus allowing simultaneous therapy and diagnosis (theranostics) [53-57].

Doxorubicin (DOX, Fig. 1) is a non-selective anthracycline and is one of the most used chemotherapeutic drugs amongst those approved by Regulatory Agencies [58]. Indeed, DOX has shown a high activity against both solid and liquid tumours, including several types of lymphoma and leukaemia, as well as breast, bladder, stomach and ovary cancers [59]. Although DOX is routinely used as an anticancer drug, its exact mechanism of action is complex and still not completely clear. According to literature, DOX has multiple molecular targets but its cytotoxic effects are essentially based on two phenomena: (a) first, one should consider the intercalation of the planar adriamycinone moiety of DOX between adjacent DNA base pairs; this intercalation interferes with the action of the enzyme topoisomerase II (TOP2), preventing the DNA double helix from being resealed and, as a consequence, stopping DNA replication and RNA transcription [60,61]; and (b) second, by producing carbon-centred radicals and reactive oxygen species (ROS); these very reactive species disrupt the cell membrane, proteins and DNA [59,60,62]. In fact, most of the side effects of DOX are associated with ROS, as is the case of cardiotoxicity. Many body tissues possess enzymes responsible for the combat of free radicals that prevent or limit tissue damage. Since the cardiac tissue has a relatively low number of these enzymes, it will be more susceptible to these reactive species [60]. Beyond cardiotoxicity, a decrease of bone marrow activity (myelosuppression) is also often observed after cancer treatment with DOX [62]. For a more detailed knowledge about the discovery, physicochemical characteristics and biological action of this drug, we recommend the readers to consult the excellent reviews that may be found in the literature about these topics [63-67].

Nanotechnology approaches for DOX delivery started several decades ago in an attempt to reduce the severe side effects often observed after its use. Indeed, Doxil ${ }^{\circledast}$ was even the first nanotherapeutic to be approved for clinical use [68]. Since then, several other DOX-based nanotherapeutics were further introduced in the market or are under clinical trials. Also, ongoing laboratory research on this topic is still very active and promising new systems may soon go from the bench to the bedside.

This review aims at showing the potential of nanomaterials in cancer treatment, mainly as drug delivery vehicles, using doxorubicin-based nanosystems as a golden example. Since the effectiveness of a nanocarrier depends on its ability to deliver the drug in the therapeutic target, the biological barriers that may interfere in this process must be considered in its design. Also, the biological and physicochemical properties of the action's site should be taken into account when targeted and/or smart nanocarriers (sensitive to environmental conditions) are developed. Due to the importance of these aspects in the design of a nanocarrier, we start by briefly reviewing them. Then, an overview of the research that has been done around DOX-based nanotherapeutics is made, highlighting the general characteristics of the various systems under study and presenting representative examples in the form of tables. Finally, an important part of the review is dedicated to the DOX-based nanotherapeutics that have advanced from laboratory experiments to clinical studies and to those that are already available for clinical use.

\section{Nanomaterial's design: physiological barriers, tumour targeting and stimuli-responsive properties}

To be successful, nanomaterials should be designed to overcome several biological barriers that may appear along their pathway inside the body. Depending on the nanomaterial's method of administration and the localization of the cells/tissues to be treated, these may include the mononuclear phagocyte system, cellular barriers, stromal barriers and cell/organelle membranes [69,70]. Beyond surpassing these difficulties, targeted nanomaterials must find their molecular targets and interact with them in an effective manner, thus being even more challenging in terms of design. A brief description of the biological barriers that may be faced by DOX-based nanotherapeutics will be described in the next paragraphs bearing in mind that their administration in the body will be intravenously.

\section{The mononuclear phagocyte system}

The mononuclear phagocyte system (or reticuloendothelial system) makes part of our immune system and mainly consists of phagocytic cells, of which the most relevant are the macrophages [70,71]. Once inside the body, nanomaterials may suffer opsonisation (Fig. 2A) by interaction with opsonins in the blood and/or tissues, thus triggering an immune response, that is, resulting in phagocytosis and clearance from the body (or, in alternative, accumulation in organs such as the lymph nodes and the spleen). The surface charge of a nanomaterial can favour protein adsorption. It is reported that negatively charged nanomaterials are less prone to opsonisation and are consequently less recognized by the phagocytic cells, thus spending more time in blood circulation. On the contrary, other reports reveal that neutral nanomaterials, as well as positively charged ones, attract phagocytic cells attention [72-75]. In addition, a common strategy to diminish opsonisation is to cover the nanomaterial's surface with a hydrophilic polymer like polyethylene glycol (PEG). Beyond preventing phagocytosis, PEG also confers a higher biocompatibility to the nanocarrier and helps to improve its solubility in aqueous environment. Moreover, PEG prevents the in vitro aggregation of nanomaterials and increases the hydrodynamic diameter of very small nanomaterials, increasing their circulation half-time [76]. In fact, the impact of PEGylation over the overall performance of nanomaterials inside the human body is very important and that is the reason why many DOX-based nanotherapeutics include PEG in their design as will be highlighted in many examples along this review.

\section{Cellular barriers and the EPR effect}

DOX is usually administrated intravenously, being able to cross the vascular-endothelium cell lining and reach most of the body tissues. However, if the idea is to use nanomaterials to avoid side effects and target specific tissues/cells, then one should have in mind that they need to go through this cellular barrier. In fact, regarding solid tumours, this is facilitated due to a phenomenon already mentioned and known by the EPR effect (Fig. 2B). The EPR effect results from an angiogenesis process that is triggered by cancer cells. These new blood vessels within the tumour are immature and possess fenestrations that, depending on the tumour type, location and environment, can have a 


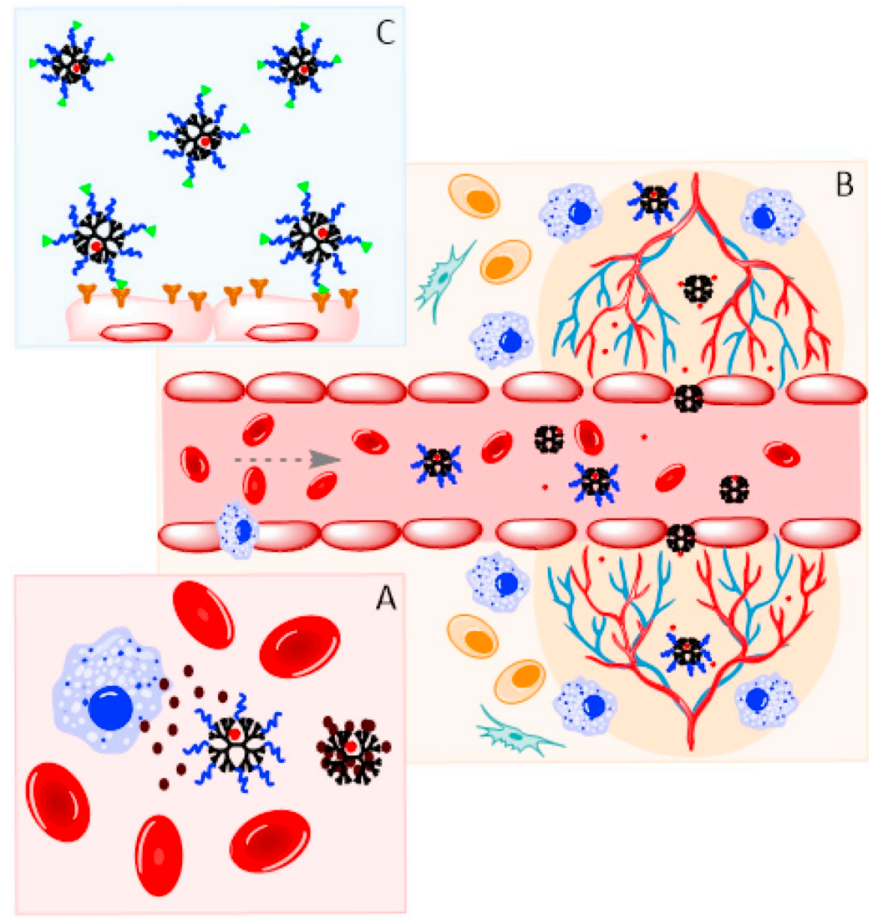

Fig. 2. A) Mononuclear phagocyte system recognition: opsonisation and phagocytosis, B) EPR effect: extravasation to tumour microenvironment through the leaky vessels and retention within the tumour tissue, and C) Active targeting: selective recognition of tumour cells through specific ligand-receptor interaction.

size ranging from 200 to $2000 \mathrm{~nm}$ [77,78]. The presence of these fenestrations associated with a lack of lymphatic drainage in the tumour site thus result in an enhanced permeability and retention effect [10]. Whereas small molecules can be re-absorbed by diffusion to the blood circulation, macromolecules or nanomaterials cannot due to their size and, for this reason, accumulate in the tumour site [79]. The EPR effect is, in fact, a passive form of targeting. This means that the targeting process depends on the characteristics of the biological system (the tumour and the new blood vessels, in this case) and not on especial properties of the nanomaterial.

Beyond extravasation due to the EPR effect, nanomaterials may be transported across the vascular-endothelium cell lining by transcytosis [80], a process that strongly depends on nanomaterial's physicochemical properties like composition, size, shape, flexibility and charge [10]. This mechanism may also be used to cross the blood-brain barrier (BBB) that is another important cellular barrier faced by a nanomaterial that is aimed at treating brain diseases. The BBB separates the circulating blood from the central nervous system and is highly selective [70]. DOX, for example, cannot cross the BBB which is a limitation for its use in the treatment of brain cancers [62]. In this case, the use of nanomaterials especially designed to serve as shuttles for DOX delivery into the brain would certainly extend the possibility of benefiting from the therapeutic properties of this drug. Several examples of DOX-based nanotherapeutics that were particularly designed to cross the BBB will appear along this review [49,81-85]. These nanomaterials were specifically designed to interact with cell surface receptors and, as such, follow an internalization mechanism known by receptor-mediated transcytosis $[86,87]$.

\section{Stromal barriers}

After crossing the cellular barriers that separate the blood from the tissues, nanomaterials may further find stromal barriers, that is, they must be transported through the interstitial space around cells to reach their target. Stromal barriers in solid tumours can be even more difficult to be crossed as the extracellular matrix produced by cancer cells is distinct from the one existent in normal tissues, being stiffer and highly heterogeneous [88]. Also, the abnormal architecture of the blood vessels in tumours and the lack of lymphatic drainage leading to a lack of perfusion can result in an increase of the fluid pressure inside the tumour that, ultimately, will retard the movement of nanomaterials [69]. In part, this phenomenon counterbalances the EPR effect. In fact, the easiness with which the nanomaterial follows its path towards the target cells will depend on the characteristics of the biological tissue but also on their own properties, e.g., size, charge, and flexibility.

\section{Cell/organelle membranes}

DOX, like other drugs, can be internalized by cells through passive diffusion and accumulates intracellularly at high concentrations which is attributed to its lipophilic properties and easy DNA intercalation [65]. On the other side, the tumour microenvironment is often characterized by a privation of oxygen and low $\mathrm{pH}$ due to the change of cell metabolism towards fermentative processes $[77,89]$. This low $\mathrm{pH}$ can affect the cellular uptake of drugs that are weak bases, as is the case of DOX. The acid environment will retain the drug outside the cells in a great extent by a process called "ion-trapping". In this context, the use of nanomaterials can help to surpass this problem, that is, to increase the cellular uptake of DOX.

Notwithstanding, the cell membrane as well as organelle membranes constitute barriers for the nanomaterials themselves (loaded, or not, with a drug). Depending on the type of cells and on their own properties, nanomaterials can enter cells by phagocytosis (a process triggered by opsonisation) or by pinocytosis. The later can further be classified in four mechanisms: clathrin-mediated endocytosis, caveolinmediated endocytosis, macropinocytosis and another class where all the other mechanisms different from the previous ones fall [90]. Then, once inside cells, nanomaterials should be able to release their therapeutic cargo near its molecular target. This means that, possibly, they will have to cross other biological membranes, such as the nuclear, lysosomal, or mitochondrial membranes. The design of a nanomaterial should, then, take all these issues into account which, by turn, are related with its specific application. In the case of DOX-based nanotherapeutics and since DOX itself is able of crossing biological membranes, the main barrier is indeed the cell membrane. So, one expects that after cell entry, the release of DOX in the cytosol will be enough to obtain a therapeutic effect. However, nanomaterials may be retained in the endolysosomal compartments and, even if DOX is there released, iontrapping may occur inside these vesicles where an acid environment exists [32,91-93]. In this case, designing nanomaterials that are capable to act as "proton-sponges" (buffers) may help to disrupt the endolysosomal membrane so that the drug can easily reach the cytosol - this is known by the "proton-sponge effect". This proton adsorption/absorption achieved by the nanomaterial may conduct to additional pumping of protons by ATPase pumps present at the endolysosomal membrane which will be followed by an influx of chloride anions in order to maintain electric neutrality. The endolysosomal vesicles will then feel an increase in osmotic pressure and will be disrupted. Examples of this strategy are also present along this review [17,94-97].

\section{Active targeting}

Nanomaterials can actively be targeted towards biological entities. Active targeting, also known as ligand-mediated targeting, relies on the use of ligands (e.g. small molecules, carbohydrates, hormones, antibodies, peptides) with specific affinity for a molecular receptor that can, for example, be localized in the surface of the cells to be treated (Fig. 2C). This recognition between the ligand and the receptor may lead to a receptor-mediated mechanism for the cellular internalization of the nanomaterial, improving its efficacy as a delivery system [10]. In 


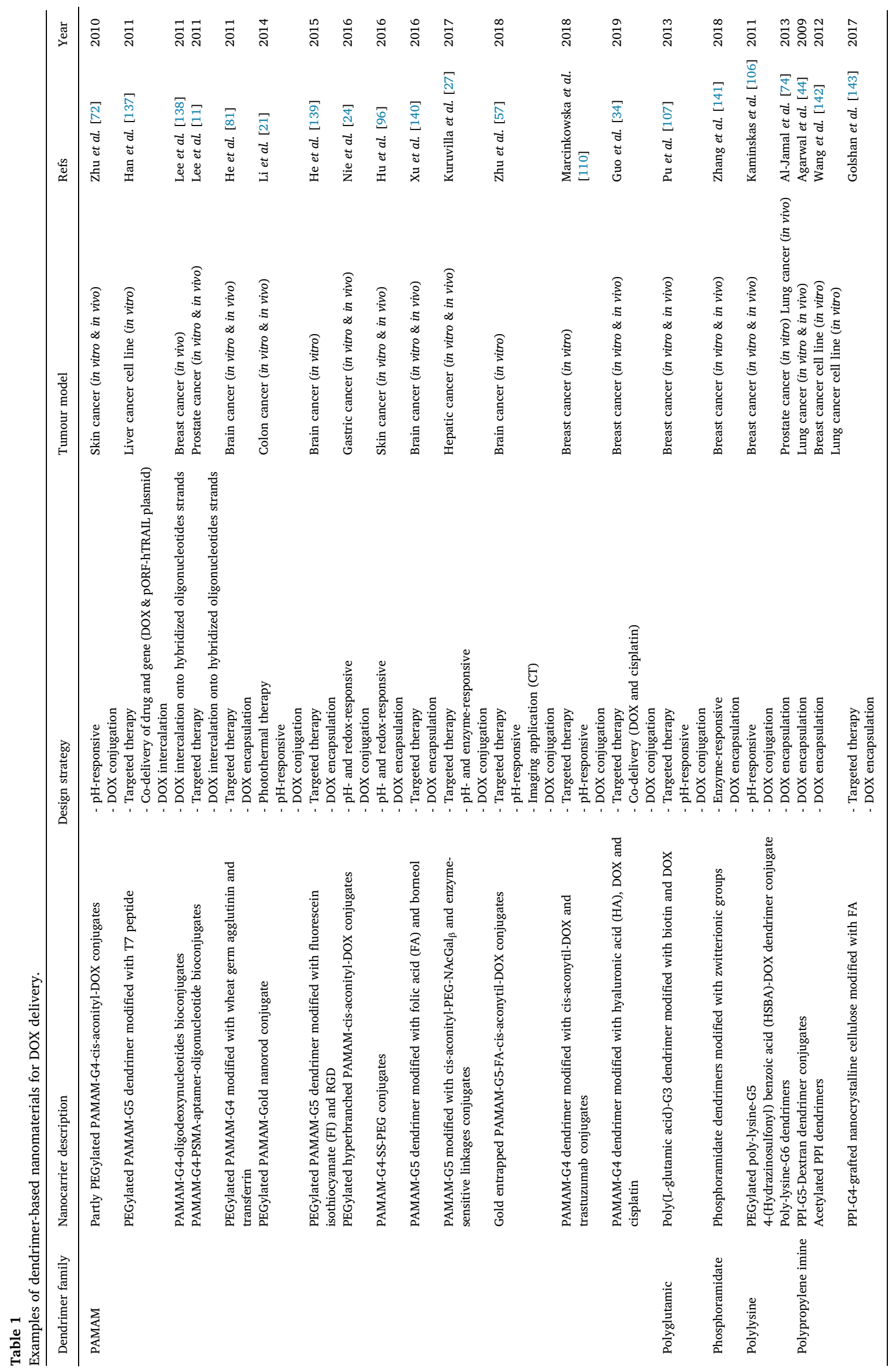


fact, the success of the process is dependent on several factors, the most important being the level of overexpression of the receptor in the target cells, and the affinity and selectivity of the ligand for the receptor $[10,98]$. In cancer therapy, active targeting has the important objective of overcoming drugs' side effects. When allied with the EPR effect (increased accumulation), active targeting approaches (increased specificity) can greatly improve the performance of nanomaterials as anticancer drug delivery vehicles [10]. The number of examples of targeted DOX-based nanotherapeutics is huge and some already moved forward towards advanced clinical trials [99-103].

\section{Stimuli-responsiveness}

Concerns about the side effects of anticancer drugs have led to the development of nanoparticles that release the drug only in the presence of specific environmental stimuli. These stimuli may be chemical or physical in nature and may correspond, for example, to a change in $\mathrm{pH}$ or temperature, or to the presence of reducing agents, specific enzymes or radiation [20,21,23-29,32,46,104,105]. Ideally, the stimulus should act only at the tumour site and may result in the release of an encapsulated drug or in chemical bonds' cleavage when the drug is covalently linked to the nanocarrier [21,24,27,57,94,106-110]. DOXbased nanotherapeutics with stimuli-responsiveness have been extensively investigated as will be shown in the next sections.

\section{Proof-of-concept studies on DOX-based nanotherapeutics}

The importance of DOX in the context of anticancer drugs justifies the large number of scientific studies that have been made in this area, as well as the variety of nanoscale systems that have been studied for its delivery in cancer cells. The following sections will review the research on DOX-based nanotherapeutics, highlighting the general characteristics, advantages and disadvantages of the different classes of nanomaterials. Representative examples of each class are shown in the form of tables with indication of the main concept behind their design.

\subsection{Polymer-based nanocarriers}

In nanomedicine, polymer-based systems are amongst the most successful nanocarriers due to their versatility. Their properties are easily tuned by playing with chemical composition, size and structure/ architecture [111]. Polymers have proven that are capable to maintain a sustained drug release of encapsulated drugs, protecting them from the surrounding environment, and of targeting cancer tissues both in passive (through the EPR effect) and active forms. Importantly, they can provide shelter to hydrophobic drugs, improving their aqueous solubility [42]. Often, they are used in combination with other classes of nanomaterials to improve their properties, as is the case of PEG that, as mentioned before, among other objectives, is usually used to camouflage nanoscale systems and avoid opsonisation (stealth nanomaterials). Also, especially by varying the chemical composition, it is possible to tune polymer's toxicity and biodegradability, both relevant aspects for nanomaterials used in medicine. For instance, poly(lactic-coglycolic acid) (PLGA) is one of the most known biodegradable and biocompatible polymers. When exposed to normal physiological conditions, PLGA is hydrolysed producing the original monomers (lactic acid and glycolic acid) that will be later metabolized through normal metabolic pathways. PLGA is considered safe and is approved by Food and Drug Administration (FDA) and European Medicines Agency (EMA, in Europe) for human use [112]. Possibly, the main disadvantage associated with polymers, that may limit their use in nanomedicine, is the difficulty in preparing molecules with well-defined sizes (they usually present a high polydispersity) and to assure homogeneity among product batches. Even so, chemical synthesis methodologies are continuously evolving and allowing, more and more, a better control over this problem.

\section{Dendrimers}

Dendrimers constitute a special group of polymers as they possess a regular and well-defined architecture, narrow polydispersity (especially when comparing them with the classical polymers) and a high number of terminal groups (multivalency) which allows further modification [113]. Typically, the basic dendrimer structure consists of a core, branched shells (their number defines the dendrimer generation) and outer functional groups [114]. Dendrimers can carry drugs by electrostatic interaction, by chemical conjugation to their surface functional groups or by encapsulation inside their inner voids [113]. Not only due to their intrinsic chemical nature, but also through the modification of their peripheral groups, it is possible to control the drug release rate in dendrimers regardless of whether the drug has been encapsulated or conjugated [115].

Table 1 presents examples of nanotherapeutics based on dendrimers and evaluated for the cellular/tumour delivery of DOX. There, one can observe that dendrimers are ideal scaffolds for the simultaneous conjugation of different chemical entities, each one serving a distinct objective. In fact, due to dendrimer's multivalency, it is possible to congregate in the same structure ligands for targeting, PEG arms, and other nanomaterials for bioimaging purposes (imaging contrast agents) or additional therapy (like those used for cancer hyperthermia), as well as the drug itself. Indeed, dendrimers are being studied for the development of theranostic materials as they can act as vehicles for drug delivery and, also, have a role in the diagnosis of diseases, and especially of cancer. For instance, several studies showed that theranostic nanomaterials based on dendrimers are able to serve as contrast agents for Magnetic Resonance Imaging (MRI) or/and for Computed Tomography (CT) imaging and/or for Positron-Emission Tomography (PET) imaging [57,116-125]. Apart from the advantages, dendrimers also present weaknesses. In particular, those dendrimers that have terminal groups with a positive charge at physiological $\mathrm{pH}$ can present a high toxicity that grows with increasing generation [126]. This is the case of poly (amidoamine) (PAMAM) dendrimers which are the dendrimers most studied until now due to their early commercial availability [127]. In this regard, acetylation may be used to decrease dendrimers' surface charge and decrease their toxicity [128-134]. Also, several works reveal that dendrimers can have a great affinity for metal ions, lipids, proteins, salts and nucleic acids, leading to disruption of biological processes and, consequently, presenting a toxicity higher than desired [135]. Naturally, the possibility of constructing dendrimers with different chemical nature also opens new routes to surpass these difficulties, as is the case of biodegradable dendrimers which are expected to offer a better performance in terms of biocompatibility. On the other hand, cationic dendrimers have been described as acting like "protonsponge materials" thus contributing for endolysome disruption and drug release into the cytosol [136].

\section{Nanogels}

Nanogels are hydrogel particles with nanoscale dimensions [144]. Research on hydrogels has become very popular since the 1960s with the interesting work of Wichterle and Lim [145]. Hydrogels are defined as three-dimensional (3D) networks made of cross-linked polymers that can absorb large amounts of water (or biological fluids) and swell still maintaining their 3D structure. Nanogels, like hydrogels, exhibit a high water content, soft consistency, flexibility and porosity [146,147]. Furthermore, they can result from physical or chemical crosslinking of natural and/or synthetic polymers, resulting in chemical stable systems, or eventually unstable by disintegration or dissolution [148]. The nanogel porosity can simply be regulated by adjusting the crosslinking density in the material which will, at the same time, control the material's affinity for water. This feature allows drug loading into the nanogel and further release with different diffusion rates depending on the drug molecule size [149]. The high biocompatibility degree and biodegradability of most hydrogels makes them especial candidates for introduction in the clinical scenario. In the meantime, many 
Table 2

Examples of nanogels for DOX delivery.

\begin{tabular}{|c|c|c|c|c|}
\hline Nanocarrier description & Design strategy & Tumour model & Refs & Year \\
\hline Acetylated HA & $\begin{array}{l}\text { - Targeted therapy } \\
\text { - DOX encapsulation }\end{array}$ & HeLa cell line (in vitro) & Park et al. [156] & 2010 \\
\hline Disulfide-core-crosslinked PEG-poly(amino acid)s star copolymers & $\begin{array}{l}\text { - Redox-responsive } \\
\text { - DOX encapsulation }\end{array}$ & HeLa cell line (in vitro) & Ding et al. [20] & 2011 \\
\hline Poly(N-isopropylacrylamide-acrylic acid) nanogel & $\begin{array}{l}\text { - Temperature- and pH-responsive } \\
\text { - DOX conjugation }\end{array}$ & $\begin{array}{l}\text { Liver cancer cell line (in } \\
\text { vitro) }\end{array}$ & Xiong et al. [157] & 2011 \\
\hline Alginate (AG) nanogel crosslinked with cystamine & $\begin{array}{l}\text { - Redox-responsive } \\
\text { - DOX encapsulation }\end{array}$ & $\begin{array}{l}\text { Bone cancer cell line (in } \\
\text { vitro) }\end{array}$ & Maciel et al. [158] & 2013 \\
\hline AG/PAMAM-G5-FI nanogels & $\begin{array}{l}\text { - Imaging therapy } \\
\text { - DOX encapsulation }\end{array}$ & $\begin{array}{l}\text { Bone cancer cell line and } \\
\text { mouse fibroblasts (in vitro) }\end{array}$ & $\begin{array}{l}\text { Gonçalves et al. } \\
\text { [159] }\end{array}$ & 2014 \\
\hline $\begin{array}{l}\text { Poly(N-isopropylacrylamide-acrylic acid) nanogels crosslinked with N,N'-bis } \\
\text { (acryloyl)cystamine or with N,N'-methylene bisacrylamide }\end{array}$ & $\begin{array}{l}\text { - Temperature-, pH- and redox- } \\
\text { responsive } \\
\text { - DOX encapsulation }\end{array}$ & $\begin{array}{l}\text { Bone cancer cell line (in } \\
\text { vitro) }\end{array}$ & Zhan et al. [22] & 2015 \\
\hline Dextrin nanogels crosslinked with formaldehyde or glyoxal & $\begin{array}{l}\text { - pH-responsive } \\
\text { - DOX encapsulation }\end{array}$ & $\begin{array}{l}\text { Colon cancer (in vitro \& in } \\
\text { vivo) }\end{array}$ & $\begin{array}{l}\text { Manchun et al. } \\
\text { [160] }\end{array}$ & 2015 \\
\hline $\begin{array}{l}\text { FA-PEG-Poly(acrylic acid) nanogels crosslinked with N,N'-bis(acryloyl) } \\
\text { cystamine }\end{array}$ & $\begin{array}{l}\text { - Targeted therapy } \\
\text { - Redox-responsive } \\
\text { - Co-delivery (DOX and cisplatin) }\end{array}$ & $\begin{array}{l}\text { Breast cancer (in vitro \& in } \\
\text { vivo) }\end{array}$ & Wu et al. [15] & 2017 \\
\hline HA-7-N,N-diethylamino-4-hydroxymethylcoumarin nanogels & $\begin{array}{l}\text { - Targeted therapy } \\
\text { - NIR- and UV-responsive } \\
\text { - DOX encapsulation }\end{array}$ & $\begin{array}{l}\text { Breast cancer cell line (in } \\
\text { vitro) }\end{array}$ & Hang et al. [161] & 2017 \\
\hline Poly(acrylic acid-4-vinylphenylboronic acid) nanogels & $\begin{array}{l}\text { - pH- and redox-responsive } \\
\text { - Co-delivery (DOX and } \\
\text { combretastatin-A4 phosphate) } \\
\text { - DOX encapsulation }\end{array}$ & $\begin{array}{l}\text { Breast cancer (in vitro \& in } \\
\text { vivo) }\end{array}$ & Yang et al. [28] & 2018 \\
\hline
\end{tabular}

nanotherapeutics based on hydrogels and used for the cellular/tumour delivery of DOX are being developed (Table 2). Smart hydrogels that respond to environmental changes (such as $\mathrm{pH}$, redox conditions and temperature, among other stimuli) have been the focus of diverse works performed both in vitro and in vivo [150-155].

\section{Polymeric micelles and polymersomes}

Drugs can be covalently linked to polymers that will then act as carriers for their delivery inside the body. Usually, the conjugated drugs are hydrophobic, and the polymer provides to the system the required solubility in aqueous environment [162]. Often, due to their amphiphilic nature, when in aqueous solution, polymer-drug conjugates tend to organize and self-assemble as micelles [163,164]. Another possibility is to form micelles using amphiphilic polymers and encapsulate the hydrophobic drug in the core of the micelle only by physical means [165]. In both situations, micelles consist of a hydrophobic core containing the drug and an outer hydrophilic shell conferring solubility in water and preventing aggregation [104]. When present at low concentrations in water, the amphiphilic building blocks of the micelles exist as discrete entities. When concentration increases above the "critical micelle concentration" (CMC), they start to self-assemble into supramolecular structures (the micelles) to maintain the hydrophobic core protected from the polar surroundings. This turning concentration point is known to depend on several experimental conditions like the polymer's chemical composition, polymer's chain length, and temperature [105]. Indeed, an important advantage of polymeric micelles is their facility of production [166,167].

Amphiphilic block-copolymers can also be used to form polymersomes by self-assembly. Polymersomes consist in vesicles that can transport a cargo in their core or within their wall [168]. Usually, the membrane of these vesicles has a thickness between 5 and $50 \mathrm{~nm}$ which may be significant compared to the polymersome size and may impact the drug's release rate.

Table 3 shows several examples of polymeric micelles and polymersomes that can be found in the literature for the specific delivery of DOX. Only by the examples presented, it is already possible to see that these systems can be very diverse in terms of chemical composition and versatile in terms of the strategy followed for drug delivery.

\subsection{Lipid-based nanocarriers}

Since the 1960s, lipid-based nanomaterials have been deeply studied as potential systems for chemical and biomedical applications [183-185]. This kind of nanomaterials became popular due to their "natural" lipid composition and therefore low toxicity [186]. There are three major lipid-based nanomaterials: solid-lipid nanoparticles (SLNs), micelles and liposomes. SLNs are usually spherical and possess a solid lipid core matrix and an outer layer of a surfactant. Lipophilic drugs can be transported in the core. Lipid components of SLNs should be solid at both body and ambient temperature and can be prepared from triglycerides, complex glyceride mixtures or even waxes [187]. The other two systems are mainly prepared from naturally occurring and/or synthetic phospholipids by self-assembly. Micelles are considered the smallest and simplest self-assembled lipid structures formed by one layer of polar lipids in aqueous solutions, forming spheres. Liposomes also consist of spherical assemblies of phospholipids that, in this case, are organized in bilayers (sometimes multiple bilayers) with a diameter size typically in the 50-200 $\mathrm{nm}$ range [188]. In aqueous solutions, the formed micelles possess a hydrophobic core, whereas liposomes possess a hydrophilic core. Since liposomes have an aqueous core and a lipid bilayer, they can accommodate both hydrophilic and hydrophobic molecules [189]. Cholesterol is generally added to the formulations of liposomes to stabilize the lipid bilayers [190].

All lipid-based nanocarriers can transport lipophilic drugs and are able to protect them from severe environmental conditions. Additional advantages are the easiness of production, possibility of functionalization and control over the drug release process $[115,191]$. Over the last years, lipid-based nanocarriers have been used as delivery vehicles for a diversity of molecules, like chemotherapeutics, enzymes, peptides, nucleic acids, antigens, antifungals and imaging agents [191,192]. Table 4 presents recent examples of research studies on lipid-based systems for the release of DOX.

\subsection{Metallic and metal oxide nanoparticles}

Nanoparticles (NPs) made of metals and metallic oxides present special properties, such as electronic, magnetic and optical, that can be tuned by adjusting their size, shape and composition [204]. Numerous 


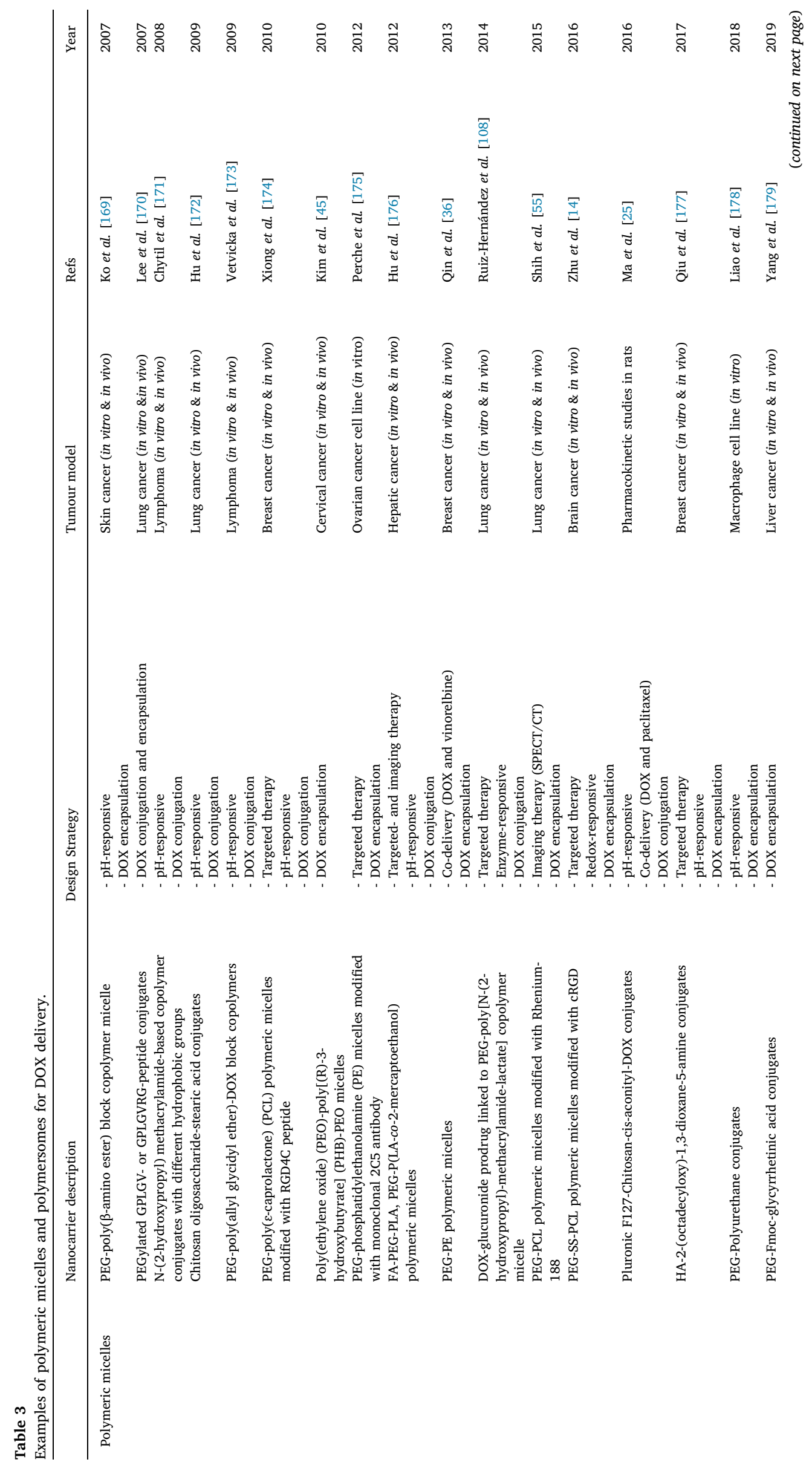


types of metallic NPs are under study for the purposes of therapy, medical imaging contrast enhancing or both (theranostic applications). Indeed, they can transport drugs adsorbed into their surface and, simultaneously, act as contrast agents in imaging techniques, like MRI (this is the case of iron oxide nanoparticles) or CT imaging (like gold nanoparticles). These NPs are also promising due to their robustness, stability, and resistance to enzymatic degradation [205]. Also, as is well-known, some metals possess antimicrobial and anti-inflammatory properties, for instance gold, silver and platinum [206]. Table 5 shows representative examples of the possible use of metallic nanoparticles for DOX delivery.

\section{Iron oxide nanoparticles}

Usually, it is not difficult to functionalize metallic NPs with different surface groups, keeping the inner properties for imaging applications. Amongst metallic NPs, those of iron oxide are quite well studied and explored due to their magnetic properties. Their size ranges from 5 to $50 \mathrm{~nm}$ and they can be easily synthesized, being possible to control their size, shape and solubility [207]. However, to achieve such stability, they need to be stabilized which is achieved by surface modification with different ligands, such as carboxylates, phosphates, and also with polymers, like PEG and polyvinyl alcohol (PVA) [208,209]. Interestingly, iron oxide NPs can be used as drug delivery systems, contrast agents in medical imaging and, in addition, by applying an adequate magnetic field, for the thermal ablation of cancer (cancer hyperthermia treatment). As naked iron oxide NPs are toxic, they are usually coated, for example with polysaccharides, PEG, and other inorganic materials. Also, in this case, the coating can confer stealth properties to the nanoparticles so that they can avoid recognition by the immune system and phagocytosis.

\section{Gold nanoparticles}

Gold nanoparticles (AuNPs) are unique amongst nanomaterials because of their inherent inert chemical properties, low toxicity, controllable size, shape and easy functionalization. The most usual method to synthesize AuNPs is through citrate reduction of chloroauric acid in water [210]. Their typical diameter is between $5-50 \mathrm{~nm}$, being their colour shape/size-dependent. The colour shifts from red to blue which can be detected in the visible part of the electromagnetic spectra [211]. The role of AuNPs in the biomedical field include labelling (for example, they can be used as contrast agents in transmission electron microscopy), drug delivery (by adsorbing drugs at their surface), heating (like iron oxide NPs, they can be used for cancer hyperthermia treatment) and sensing (due to their optoelectronic properties). Furthermore, AuNPs can attenuate X-rays and, so, are being investigated to be used as contrast agents in CT imaging. Very important is also the fact that they are very easily functionalized at the surface through the reaction of gold with sulfhydryl (R-SH) groups present in organic or biological molecules.

\section{Silver nanoparticles}

Silver nanoparticles (AgNPs) are being used in our daily life in a wide range of fields, including food, healthcare, and industry [212]. AgNPs exhibit special features such as optical, thermal, electrical and biological properties. One important characteristic is their strong antimicrobial and antifungicidal activity. Due to this property, they have been employed in several materials for medical care, namely in silicon catheters, sterilizing filters, sutures and, also, as medicines for dermatitis. Recently, AgNPs have also been studied as anticancer agents themselves, beyond the possibility of being used as drug carriers. Like AuNPs, they may be applied as diagnostic or probing mediators [213,214].

\subsection{Carbon-based nanomaterials}

Beyond their technological applications, carbon-based nanomaterials are also being explored in the biomedical field [237,238]. 
Table 4

Examples of lipid-based nanomaterials with DOX*.

\begin{tabular}{|c|c|c|c|c|c|}
\hline Lipid family & Nanocarrier description & Design Strategy & Tumour model & Refs & Year \\
\hline \multirow[t]{4}{*}{ Solid lipid NP } & PEG-PE conjugates modified with transferrin & $\begin{array}{l}\text { - Targeted therapy } \\
\text { - Co-delivery (DOX and EGFP- } \\
\text { encoding plasmid) } \\
\text { - DOX encapsulation }\end{array}$ & Lung cancer (in vitro \& in vivo) & Han et al. [193] & 2014 \\
\hline & $\begin{array}{l}\text { Glyceryl mono stearate and soya-lecithin based } \\
\text { SLNs modified with Fluorescein isothiocyanate } \\
\text { (FITC) and galactose }\end{array}$ & $\begin{array}{l}\text { - Targeted therapy } \\
\text { - DOX encapsulation }\end{array}$ & $\begin{array}{l}\text { Lung cancer (in vitro) } \\
\text { Pharmacokinetic and biodistribution } \\
\text { studies in rats }\end{array}$ & Jain et al. [194] & 2015 \\
\hline & $\begin{array}{l}\text { DSPE-PEG, DPPC, cocoa butter and palmitic acid } \\
\text { based SLNs modified with aprotinin and } \\
\text { melanotransferrin antibody }\end{array}$ & $\begin{array}{l}\text { - Targeted therapy } \\
\text { - DOX encapsulation }\end{array}$ & Brain cancer (in vitro) & Kuo et al. [84] & 2016 \\
\hline & Poloxamer 470 and precirol ATO 5 based SLNs & - DOX encapsulation & Melanoma cancer (in vitro \& in vivo) & Tupal et al. [195] & 2016 \\
\hline \multirow[t]{5}{*}{ Micelle } & $\begin{array}{l}\text { Labrafac WL } 1349^{\oplus} \text { and Solutol HS } 15^{\oplus} \text { based } \\
\text { micelles }\end{array}$ & $\begin{array}{l}\text { - Co-delivery (DOX and docetaxel) } \\
\text { - DOX encapsulation }\end{array}$ & N/A & Vrignaud et al. [35] & 2011 \\
\hline & $\begin{array}{l}\text { 1-palmitoyl-2-azelaoyl-sn-glycero-3- } \\
\text { phosphocholine based micelles }\end{array}$ & $\begin{array}{l}\text { - DOX encapsulation and } \\
\text { complexation }\end{array}$ & Blood cancer (in vitro) & Wang et al. [196] & 2012 \\
\hline & $\begin{array}{l}\text { PEG, PE, DC-Cholesterol and DOPE based micelles } \\
\text { containing manganese NPs }\end{array}$ & $\begin{array}{l}\text { - Imaging therapy (MRI) } \\
\text { - Co-delivery (DOX and DNA) } \\
\text { - DOX encapsulation }\end{array}$ & $\begin{array}{l}\text { Kidney and lung cancer (in vitro \& in } \\
\text { vivo) }\end{array}$ & Howell et al. [54] & 2013 \\
\hline & $\begin{array}{l}\text { DSPE-PEG based micelles modified with CRGDK } \\
\text { peptide }\end{array}$ & $\begin{array}{l}\text { - Targeted therapy } \\
\text { - DOX encapsulation }\end{array}$ & Breast cancer (in vitro \& in vivo) & Wei et al. [47] & 2013 \\
\hline & $\begin{array}{l}\text { DSPE-PEG based micelles modified with GE11 } \\
\text { peptide }\end{array}$ & $\begin{array}{l}\text { - Targeted therapy } \\
\text { - DOX encapsulation }\end{array}$ & Breast cancer (in vitro) & Fan et al. [13] & 2016 \\
\hline \multirow[t]{12}{*}{ Liposome } & $\begin{array}{l}\text { HSPC/Cholesterol and DSPE-PEG based liposomes } \\
\text { modified with Fab' } 2 \text { 22-1D8 antibody fragment }\end{array}$ & $\begin{array}{l}\text { - Targeted therapy } \\
\text { - DOX encapsulation }\end{array}$ & Fibrosarcoma (in vitro \& in vivo) & $\begin{array}{l}\text { Hatakeyama et al. } \\
\text { [197] }\end{array}$ & 2007 \\
\hline & $\begin{array}{l}\text { DSPE-PEG and DPPC or HSPC based liposomes } \\
\text { modified } \\
\text { with HER2-antibody }\end{array}$ & $\begin{array}{l}\text { - Targeted therapy } \\
\text { - Light-responsive } \\
\text { - Co-delivery (DOX and hollow } \\
\text { gold nanospheres) } \\
\text { - DOX encapsulation }\end{array}$ & $\begin{array}{l}\text { Carcinoma and lung cancer (in vitro \& } \\
\text { in vivo) }\end{array}$ & Li et al. [198] & 2015 \\
\hline & $\begin{array}{l}\text { HSPC/Cholesterol based liposomes modified with } \\
\text { sialic acid-octadecylamine }\end{array}$ & $\begin{array}{l}\text { - Targeted therapy } \\
\text { - Co-delivery (DOX and } \\
\text { dexamethasone palmitate) } \\
\text { - DOX encapsulation }\end{array}$ & Sarcoma cancer (in vivo) & Sun et al. [38] & 2016 \\
\hline & $\begin{array}{l}\text { DSPE-PEG based liposomes modified with GE11 } \\
\text { peptide and cetuximab Fab' antibody fragment }\end{array}$ & $\begin{array}{l}\text { - Targeted therapy } \\
\text { - Temperature responsive } \\
\text { - DOX encapsulation }\end{array}$ & Breast cancer cell line (in vitro) & Haeri et al. [199] & 2016 \\
\hline & $\begin{array}{l}\text { DPPC/DSPC/Cholesterol/DSPE-PEG based } \\
\text { liposomes }\end{array}$ & $\begin{array}{l}\text { - Imaging therapy (MRI) } \\
\text { - Ultrasound responsive } \\
\text { - Co-delivery (DOX and } \\
\text { gadoteridol) } \\
\text { - DOX encapsulation }\end{array}$ & Breast cancer (in vitro \& in vivo) & Rizzitelli et al. [56] & 2016 \\
\hline & $\begin{array}{l}\text { DSPC/CH; DSPC/DOTAP/Cholesterol and DSPC/ } \\
\text { PEG-DSPE/ DOTAP/Cholesterol based liposomes }\end{array}$ & $\begin{array}{l}\text { - Co-delivery (DOX and } \\
\text { - 5-Flurouracil) } \\
\text { - DOX encapsulation }\end{array}$ & Breast cancer (in vitro \& in vivo) & Camacho et al. [39] & 2016 \\
\hline & $\begin{array}{l}\text { DSPE-PEG based liposomes modified with } \mathrm{H}_{7} \mathrm{~K}\left(\mathrm{R}_{2}\right)_{2} \\
\text { peptide }\end{array}$ & $\begin{array}{l}\text { - Targeted therapy } \\
\text { - pH responsive } \\
\text { - DOX encapsulation }\end{array}$ & Brain cancer (in vitro \& in vivo) & Zhao et al. [49] & 2016 \\
\hline & $\mathrm{DPPC} / \mathrm{DSPC} / \mathrm{DPPG}_{2}$ based liposomes & $\begin{array}{l}\text { - Imaging therapy (MRI) } \\
\text { - Temperature responsive } \\
\text { - Co-delivery (DOX and } \\
\text { gadoteridol) } \\
\text { - DOX encapsulation }\end{array}$ & Sarcoma cancer (in vitro \& in vivo) & Peller et al. [200] & 2016 \\
\hline & PEG-DSPE/DPPC/Cholesterol based liposomes & $\begin{array}{l}\text { - Co-delivery (DOX and curcumin) } \\
\text { - DOX encapsulation }\end{array}$ & Colon cancer cell line (in vitro) & $\begin{array}{l}\text { Sesarman et al. } \\
\text { [201] }\end{array}$ & 2017 \\
\hline & $\begin{array}{l}\text { DOTAP/DSPE-PEG and POPC/Cholesterol/DSPE- } \\
\text { PEG based liposomes }\end{array}$ & $\begin{array}{l}\text { - Co-delivery (DOX-DNA aptamer } \\
\text { complex and tobramycin } \\
\text { - DOX-DNA aptamer complex } \\
\text { encapsulation }\end{array}$ & HeLa cancer cell line (in vitro) & Plourde et al. [202] & 2017 \\
\hline & $\begin{array}{l}\text { Lecithin S100/DOTAP/Cholesterol based liposomes } \\
\text { modified with selenium }\end{array}$ & - DOX encapsulation & Lung cancer (in vitro \& in vivo) & Xie et al. [203] & 2018 \\
\hline & $\begin{array}{l}\text { DSPE-PEG based liposomes modified with D- } \\
\text { mannose or } \\
\text { L-fucose }\end{array}$ & $\begin{array}{l}\text { - Targeted therapy } \\
\text { - DOX encapsulation }\end{array}$ & Sarcoma cancer (in vitro \& in vivo) & Li et al. [19] & 2019 \\
\hline
\end{tabular}

* DSPE: 1,2-Distearoyl-sn-glycero-3-phosphoethanolamine; HER2: Human epidermal growth factor receptor-2; HSPC: Hydrogenated soy phosphatidylcholine; DPPC: 1.2-Dipalmitoyl-sn-glycero-3-phosphocoline; DSPC: 1.2-Distearoyl-sn-glycero-3-phosphocoline; DC: 3ß-[N-(N', N'-dimethylaminoethane)-carbamoyl]; DOPE: dioleoylphosphatidyl-ethanolamine; DOTAP: 1,2-dioleoyl-3- trimethylammonium-propane; DPPG $_{2}$ : 1,2-dipalmitoyl-sn-glycero-3-phosphodiglycerol; POPC: 1-palmitoyl-2- oleoyl-sn-glycero-3-phosphocholine

Important examples are graphene, carbon nanotubes (CNTs) and the fullerene $\mathrm{C}_{60}$. Graphene consists in a single layer sheet structure with a thickness of a carbon atom, CNTs possess cylindrical hollow structures with the walls also with a thickness of a carbon atom, and $\mathrm{C}_{60}$ is a spherical molecule having 60 carbon atoms and the shape of a soccer ball. These three kinds of nanomaterials can be easily functionalized to increase their solubility in water, to allow their use as drug carriers and to tune their interaction with biological targets [239]. Currently, other 
Table 5

Examples of metallic-based NPs with DOX.

\begin{tabular}{|c|c|c|c|c|c|}
\hline Metal family & Nanocarrier description & Design strategy & Tumour model & Refs & Year \\
\hline \multirow[t]{12}{*}{ Iron Oxide } & Gelatin-coated iron oxide NPs & - DOX encapsulation & N/A & Gaihre et al. [215] & 2009 \\
\hline & Poly-n-isopropylacrylamide-coated iron oxide NPs & $\begin{array}{l}\text { - Temperature responsive } \\
\text { - DOX encapsulation }\end{array}$ & Liver cancer (in vitro \& in vivo) & $\begin{array}{l}\text { Purushotham et al. } \\
\text { [216] }\end{array}$ & 2009 \\
\hline & Polyvinyl alcohol (PVA)-coated iron oxide NPs & - DOX encapsulation & N/A & Kayal et al. [217] & 2010 \\
\hline & $\begin{array}{l}\text { PEG-trimellitic anhydride chloride polymer modified with FA } \\
\text { and iron oxide NPs }\end{array}$ & $\begin{array}{l}\text { - Targeted therapy } \\
\text { - Imaging therapy (MRI) } \\
\text { - DOX encapsulation }\end{array}$ & Liver cancer (in vitro \& in vivo) & Maeng et al. [218] & 2010 \\
\hline & $\begin{array}{l}\text { PEGylated iron-platinum/iron oxide core-shell NPs modified } \\
\text { with FA }\end{array}$ & $\begin{array}{l}\text { - Targeted therapy } \\
\text { - Imaging therapy (MRI) } \\
\text { - DOX encapsulation }\end{array}$ & Breast cancer (in vitro \& in vivo) & Liu et al. [219] & 2013 \\
\hline & $\begin{array}{l}\text { PEG-poly(4-vinylbenzylphosphonate) polymer-coated iron } \\
\text { oxide NPs }\end{array}$ & - DOX encapsulation & Colon cancer (in vitro \& in vivo) & $\begin{array}{l}\text { Hałupka-Bryl et al. } \\
\text { [220] }\end{array}$ & 2014 \\
\hline & PEGylated mesoporous silica-coated iron oxide NPs & - DOX encapsulation & N/A & $\begin{array}{l}\text { Pourjavadi et al. } \\
\text { [221] }\end{array}$ & 2015 \\
\hline & HA coated-iron oxide NPs & - DOX encapsulation & Breast cancer cell line (in vitro) & Aval et al. [222] & 2016 \\
\hline & PVA coated-iron oxide NPs & - DOX conjugation & N/A & Nadeem et al. [223] & 2016 \\
\hline & Heparin-coated iron oxide NPs & $\begin{array}{l}\text { - Imaging therapy (MRI) } \\
\text { - DOX conjugation }\end{array}$ & Lung cancer (in vitro \& in vivo) & Yang et al. [224] & 2016 \\
\hline & $\begin{array}{l}\text { Iron oxide NPs coated with PEG-polydopamine polymer } \\
\text { crosslinked with N,N-Bis(acryloyl) }\end{array}$ & $\begin{array}{l}\text { - Redox responsive } \\
\text { - DOX encapsulation }\end{array}$ & $\mathrm{N} / \mathrm{A}$ & Shang et al. [26] & 2017 \\
\hline & Pluronic F127-coated iron oxide NPs & - DOX encapsulation & Brain cancer cell line (in vitro) & Mdlovu et al. [225] & 2019 \\
\hline \multirow[t]{14}{*}{ Gold } & $\begin{array}{l}\text { AuNPs stabilized with P(LA-DOX)- } b \text {-PEG-OH polymer } \\
\text { modified with FA }\end{array}$ & $\begin{array}{l}\text { - Targeted therapy } \\
\text { - pH responsive } \\
\text { - DOX conjugation }\end{array}$ & Breast cancer cell line (in vitro) & $\begin{array}{l}\text { Prabaharam et al. } \\
\text { [226] }\end{array}$ & 2009 \\
\hline & Gold nanoclusters modified with FA & $\begin{array}{l}\text { - Targeted and imaging } \\
\text { therapy } \\
\text { - Light responsive } \\
\text { - Co-delivery (DOX and MPA) } \\
\text { - DOX conjugation }\end{array}$ & $\begin{array}{l}\text { Lung, liver, breast and colon } \\
\text { cancer cells (in vitro \& in vivo) }\end{array}$ & Chen et al. [227] & 2012 \\
\hline & Multifunctional gold nanorods & $\begin{array}{l}\text { - Targeted and imaging (PET) } \\
\text { therapy } \\
\text { - pH responsive } \\
\text { - Co-delivery (DOX and }{ }^{64} \mathrm{Cu} \\
\text { chelator) } \\
\text { - DOX conjugation }\end{array}$ & Brain cancer (in vitro \& in vivo) & Xiao et al. [53] & 2012 \\
\hline & DOX-CPLGLAGG peptide AuNPs conjugates & $\begin{array}{l}\text { - Targeted therapy } \\
\text { - Redox and enzyme } \\
\text { responsive } \\
\text { - DOX conjugation }\end{array}$ & $\begin{array}{l}\text { Mouse head and neck } \\
\text { carcinoma cell line (in vitro \& } \\
\text { in vivo) }\end{array}$ & Chen et al. [228] & 2013 \\
\hline & PEG-AuNPs conjugates & - DOX conjugation & Breast cancer (in vitro \& in vivo) & Sun et al. [229] & 2014 \\
\hline & $\begin{array}{l}\text { PLGA-core gold-shell conjugates modified with human serum } \\
\text { albumin (HSA)-indocyanine green-FA }\end{array}$ & $\begin{array}{l}\text { - Targeted therapy } \\
\text { - DOX encapsulation }\end{array}$ & Breast cancer (in vitro \& in vivo) & Topete et al. [230] & 2014 \\
\hline & PEGylated magnetic AuNPs & - DOX encapsulation & Breast cancer (in vitro \& in vivo) & Elbialy et al. [231] & 2015 \\
\hline & $\begin{array}{l}\text { AuNPs crosslinked with } \\
\text { PCL-Poly(2-(dimethylamino) ethylmethacrylate)-PEG }\end{array}$ & - DOX encapsulation & $\begin{array}{l}\text { Mouse head and neck } \\
\text { carcinoma cell line (in vitro \& } \\
\text { in vivo) }\end{array}$ & Jeon et al. [232] & 2015 \\
\hline & $\begin{array}{l}\text { PEGylated } \\
\text { 3-[2-Pyridyldithio]propionyl hydrazide-AuNP conjugates }\end{array}$ & $\begin{array}{l}\text { - } \mathrm{pH} \text { responsive } \\
\text { - DOX conjugation }\end{array}$ & $\begin{array}{l}\text { Human head and neck } \\
\text { squamous carcinoma cell line } \\
\text { (in vitro) }\end{array}$ & Lee et al. [109] & 2015 \\
\hline & AuNPs-DOX conjugate & - DOX conjugation & Skin cancer (in vitro \& in vivo) & Zhang et al. [233] & 2015 \\
\hline & Oligonucleotide-conjugated AuNPs & $\begin{array}{l}\text { - Targeted therapy } \\
\text { - DOX complexation }\end{array}$ & Colon cancer (in vitro \& in vivo) & Lee et al. [234] & 2017 \\
\hline & Polyvinylpyrrolidone-coated AuNPs & - DOX conjugation & Lung cancer (in vitro) & $\begin{array}{l}\text { Ramalingam et al. } \\
\text { [235] }\end{array}$ & 2018 \\
\hline & PEGylated gold nanocages modified with biotin & $\begin{array}{l}\text { - Targeted therapy } \\
\text { - Light responsive } \\
\text { - Co-delivery (DOX, quercetin } \\
\text { and tetradecanol) } \\
\text { - DOX encapsulation }\end{array}$ & Breast cancer cell line (in vitro) & Zhang et al. [18] & 2018 \\
\hline & $\begin{array}{l}\text { Oxidized HA-decorated dihydroxyphenyl/hydrazide } \\
\text { bifunctionalized hydroxyethyl CHI-gold nanorods conjugates }\end{array}$ & $\begin{array}{l}\text { - Targeted therapy } \\
\text { - pH responsive } \\
\text { - DOX encapsulation }\end{array}$ & Breast cancer cell line (in vitro) & Hou et al. [236] & 2019 \\
\hline Silver & Alendronate-coated AgNPs & $\begin{array}{l}\text { - pH responsive } \\
\text { - Co-delivery (DOX and } \\
\text { alendronate) } \\
\text { - DOX conjugation }\end{array}$ & HeLa cancer cell line (in vitro) & Benyettou et al. [23] & 2015 \\
\hline
\end{tabular}

carbon nanomaterials are also being tested in the biomedical area, like carbon nanohorns, derived from CNTs but having a conical cap, and carbon dots which are fluorescent nanomaterials. Curiously, many of the mentioned carbon structures were tested for drug delivery applications and, particularly, for the delivery of DOX as can be seen in the examples listed in Table 6.

\subsection{Clay-based nanomaterials}

Clay-based nanomaterials are already being used for cosmetic and pharmaceutic applications and, so, researchers are now trying to take advantage of their properties for further application in tissue engineering, regenerative medicine, medical imaging and cancer 
Table 6

Examples of carbon-based nanomaterials with DOX.

\begin{tabular}{|c|c|c|c|c|c|}
\hline & Nanocarrier description & Design Strategy & Tumour model & Refs & Year \\
\hline Nanohorns & $\begin{array}{l}\text { PEGylated oxidized carbon single-walled } \\
\text { nanohorns }\end{array}$ & - DOX conjugation & Lung cancer cell line (in vitro) & $\begin{array}{l}\text { Murakami et al. } \\
{[240]}\end{array}$ & 2006 \\
\hline \multirow[t]{9}{*}{ Carbon Nanotubes } & Pluronics F127-coated multi-walled CNTs & - DOX encapsulation & Breast cancer cell line (in vitro) & $\begin{array}{l}\text { Ali-Boucetta et al. } \\
\text { [241] }\end{array}$ & 2008 \\
\hline & $\begin{array}{l}\text { AG and CHI-coated single-walled CNTs modified } \\
\text { with FA }\end{array}$ & $\begin{array}{l}\text { - Targeted therapy } \\
\text { - pH responsive } \\
\text { - DOX encapsulation }\end{array}$ & HeLa cancer cell line (in vitro) & Zhang et al. [242] & 2009 \\
\hline & PEGylated single-walled CNTs & - DOX encapsulation & Lymphoma (in vitro \& in vivo) & Liu et al. [243] & 2009 \\
\hline & PEGylated single-walled CNTs & $\begin{array}{l}\text { - } \mathrm{pH} \text { responsive } \\
\text { - DOX conjugation }\end{array}$ & Lung cancer cell line (in vitro) & Gu et al. [244] & 2011 \\
\hline & Multi-walled CNTs modified with FA and iron NPs & $\begin{array}{l}\text { - Targeted therapy } \\
\text { - Light responsive } \\
\text { - DOX encapsulation }\end{array}$ & HeLa cancer cell line (in vitro) & Li et al. [245] & 2011 \\
\hline & $\begin{array}{l}\text { PEGylated oxidized multi-walled CNTs modified } \\
\text { with angiopep-2 }\end{array}$ & $\begin{array}{l}\text { - Targeted therapy } \\
\text { - DOX encapsulation }\end{array}$ & Brain cancer (in vitro \& in vivo) & Ren et al. [83] & 2012 \\
\hline & PEGylated multi-walled CNTs modified with FA & $\begin{array}{l}\text { - Targeted therapy } \\
\text { - DOX encapsulation }\end{array}$ & $\begin{array}{l}\text { HeLa cancer cell line (in vitro) } \\
\text { Liver perfusion study (in vivo) }\end{array}$ & Dinan et al. [246] & 2014 \\
\hline & $\begin{array}{l}\text { Oxidized multi-walled CTNs modified with } \\
\text { galactosylated CHI }\end{array}$ & $\begin{array}{l}\text { - Targeted therapy } \\
\text { - } \mathrm{pH} \text { responsive } \\
\text { - DOX encapsulation }\end{array}$ & Liver cancer (in vitro \& in vivo) & Qi et al. [247] & 2015 \\
\hline & Carbon dots functionalized with gold nanorods & $\begin{array}{l}\text { - Imaging therapy } \\
\text { - Temperature responsive } \\
\text { - DOX conjugation }\end{array}$ & $\begin{array}{l}\text { Vero cell line and breast cancer cell line } \\
\text { (in vitro) }\end{array}$ & Pandey et al. [248] & 2013 \\
\hline \multirow[t]{4}{*}{ Fullerene } & $\mathrm{C}_{60}$ & - DOX conjugation & Breast cancer cell line (in vitro) & Liu et al. [249] & 2010 \\
\hline & $\mathrm{C}_{60}$ & - DOX conjugation & Chicken embryo (in vitro \& in vivo) & $\begin{array}{l}\text { Blazkova et al. } \\
\text { [250] }\end{array}$ & 2014 \\
\hline & $\mathrm{C}_{60}$-Polyethylenimine (PEI) conjugates & $\begin{array}{l}\text { - Photodynamic therapy } \\
\text { - pH responsive } \\
\text { - DOX conjugation }\end{array}$ & Skin cancer (in vitro and in vivo) & Shi et al. [251] & 2014 \\
\hline & PEGylated $\mathrm{C}_{60}$ & - DOX conjugation & Breast cancer cell line (in vitro) & $\begin{array}{l}\text { Magoulas et al. } \\
\text { [252] }\end{array}$ & 2015 \\
\hline \multirow[t]{8}{*}{ Carbon dots } & $\begin{array}{l}\text { Carbon dots modified with FA and bovine serum } \\
\text { albumin (BSA) }\end{array}$ & $\begin{array}{l}\text { - Targeted therapy } \\
\text { - DOX encapsulation }\end{array}$ & $\begin{array}{l}\text { Vero cell line and HeLa cancer cell line } \\
\text { (in vitro) }\end{array}$ & $\begin{array}{l}\text { Mewada et al. } \\
\text { [253] }\end{array}$ & 2014 \\
\hline & Carbon dots modified with transferrin & $\begin{array}{l}\text { - Targeted therapy } \\
\text { - DOX conjugation }\end{array}$ & Brain cancer cell line (in vitro) & Li et al. [85] & 2016 \\
\hline & Carbon dots modified with heparin & $\begin{array}{l}\text { - pH responsive } \\
\text { - Co-delivery (DOX and } \\
\text { heparin) } \\
\text { - DOX encapsulation }\end{array}$ & $\begin{array}{l}\text { Breast and lung cancer cell line (in vitro) } \\
\text { HeLa cancer cell line (in vitro) }\end{array}$ & Zhang et al. [254] & 2017 \\
\hline & Polydopamine-coated carbon dots & - DOX encapsulation & HeLa cancer cell line (in vitro) & Sun et al. [255] & 2017 \\
\hline & Carbon dots modified with PEI and HA & $\begin{array}{l}\text { - Targeted therapy } \\
\text { - DOX conjugation }\end{array}$ & $\begin{array}{l}\text { Mouse fibroblast cell line (in vitro) } \\
\text { HeLa cancer cell line (in vitro) }\end{array}$ & Gao et al. [256] & 2017 \\
\hline & Carbon dots & - DOX conjugation & Breast cancer cell line (in vitro) & Kong et al. [257] & 2018 \\
\hline & $\begin{array}{l}\text { Magnetic hollow }\left(\mathrm{Fe}_{3} \mathrm{O}_{4}\right) \text { and porous carbon NPs } \\
\text { modified with poly }(\gamma \text {-glutamic acid }) \text { and FA }\end{array}$ & $\begin{array}{l}\text { - Targeted and imaging } \\
\text { therapy } \\
\text { - Temperature, redox and } \mathrm{pH} \\
\text { responsive } \\
\text { - DOX encapsulation }\end{array}$ & $\begin{array}{l}\text { HeLa cancer cell line (in vitro) } \\
\text { Biodistribution studies (in vivo) }\end{array}$ & Wu et al. [258] & 2018 \\
\hline & $\begin{array}{l}\text { Carbon dots modified with catechol-borane } \\
\text { moieties }\end{array}$ & $\begin{array}{l}\text { - } \mathrm{pH} \text { responsive } \\
\text { - DOX encapsulation }\end{array}$ & HeLa cancer cell line (in vitro) & Wu et al. [259] & 2019 \\
\hline
\end{tabular}

treatment [260]. Especial attention is being given to the synthetic clay Laponite ${ }^{\circledast}\left(\mathrm{Na}_{0.7}^{+}\left[\left(\mathrm{Si}_{8} \mathrm{Mg}_{5.5} \mathrm{Li}_{0.3}\right) \mathrm{O}_{20}(\mathrm{OH})_{4}\right]^{-0.7}\right.$, LP) that can be produced with a controllable composition at a large scale and low cost. Although there are several different LP grades commercially available, for medical applications, the grades having high purity and low heavy metals content should be used [261]. LP is composed of nanoscale crystals with a disk shape (about $25 \mathrm{~nm}$ in diameter and $0.92 \mathrm{~nm}$ in thickness). In these disks, the faces are negatively charged whereas the

Table 7

Examples of clay-based nanomaterials with DOX.

\begin{tabular}{|c|c|c|c|c|}
\hline Nanocarrier description & Design strategy & Tumour model & Refs & Year \\
\hline LP nanodisks & - DOX encapsulation & Liver cancer (in vivo) & Li et al. [265] & 2014 \\
\hline LP nanodisks modified with PEG-PLA polymer & - DOX encapsulation & Bone cancer (in vitro) & Wang et al. [92] & 2014 \\
\hline Alginate-coated LP nanodisks & - DOX encapsulation & Bone cancer (in vitro) & Gonçalves et al. [32] & 2014 \\
\hline PEGylated LP nanodisks modified with lactobionic acid & $\begin{array}{l}\text { - Targeted therapy } \\
\text { - DOX encapsulation }\end{array}$ & Liver cancer (in vitro) & Chen et al. [95] & 2015 \\
\hline $\begin{array}{l}\text { Poly(allylamine) hydrochloride (PAH)/ poly(sodiumstyrene sulfonate) (PSS)-coated } \\
\text { LP nanohybrids }\end{array}$ & - DOX encapsulation & Breast cancer (in vitro) & Xiao et al. [105] & 2016 \\
\hline LP nanodisks modified with PEG-PLA, PEI, AuNPs and HA & $\begin{array}{l}\text { - Targeted therapy } \\
\text { - DOX encapsulation }\end{array}$ & $\begin{array}{l}\text { HeLa cancer cell line (in vitro \& in } \\
\text { vivo) }\end{array}$ & Zhuang et al. [17] & 2017 \\
\hline LP nanodisks modified with HA & $\begin{array}{l}\text { - Targeted therapy } \\
\text { - DOX encapsulation }\end{array}$ & HeLa cancer cell line (in vitro) & Jiang et al. [97] & 2019 \\
\hline
\end{tabular}


Table 8

Examples of biological-based nanomaterials with DOX.

\begin{tabular}{|c|c|c|c|c|c|}
\hline Biological family & Nanocarrier description & Design strategy & Tumour model & Refs & Year \\
\hline \multirow[t]{7}{*}{ Albumin } & HSA NPs & - DOX encapsulation & Brain cancer cell line (in vitro) & Dreis et al. [267] & 2007 \\
\hline & $\begin{array}{l}\text { HSA NPs modified with DI17E6 monoclonal } \\
\text { antibody }\end{array}$ & $\begin{array}{l}\text { - Targeted therapy } \\
\text { - DOX encapsulation }\end{array}$ & Skin cancer cell line (in vitro) & $\begin{array}{l}\text { Wagner et al. } \\
\text { [268] }\end{array}$ & 2010 \\
\hline & PEGylated modified HSA NPs modified with RGD & $\begin{array}{l}\text { - Targeted therapy } \\
\text { - DOX encapsulation }\end{array}$ & Skin cancer cell line (in vitro) & Xu et al. [269] & 2011 \\
\hline & HSA NPs modified with TRAIL ligand and transferrin & $\begin{array}{l}\text { - Targeted therapy } \\
\text { - DOX encapsulation }\end{array}$ & $\begin{array}{l}\text { Pancreatic and breast cancer cell line } \\
\text { (in vitro) } \\
\text { Colon cancer (in vitro \& in vivo) }\end{array}$ & Bae et al. [270] & 2012 \\
\hline & $\begin{array}{l}\text { HSA NPs modified with palmitoyl-poly-arginine } \\
\text { peptides }\end{array}$ & $\begin{array}{l}\text { - Targeted therapy } \\
\text { - DOX conjugation }\end{array}$ & HeLa cancer cell line (in vitro) & $\begin{array}{l}\text { Ichimizu et al. } \\
\text { [271] }\end{array}$ & 2018 \\
\hline & BSA NPs & $\begin{array}{l}\text { - Co-delivery (DOX and } \\
\text { cyclopamine) } \\
\text { - DOX encapsulation }\end{array}$ & Breast cancer (in vitro \& in vivo) & Lu et al. [52] & 2019 \\
\hline & HSA NPs & - DOX encapsulation & Colon cancer (in vitro \& in vivo) & Kimura et al. [272] & 2019 \\
\hline \multirow[t]{5}{*}{ Exosomes } & $\begin{array}{l}\text { DOX-loaded exosomes and exosome-mimetic } \\
\text { nanovesicles obtained from macrophages and lung } \\
\text { cell line }\end{array}$ & - DOX encapsulation & $\begin{array}{l}\text { HUVEC cell line (in vitro) } \\
\text { Colon cell line (in vitro \& in vivo) }\end{array}$ & Jang et al. [273] & 2013 \\
\hline & $\begin{array}{l}\text { DOX-loaded exosomes obtained from immature } \\
\text { dendritic cells labelled with iRGD-Lamp2b protein }\end{array}$ & $\begin{array}{l}\text { - Targeted therapy } \\
\text { - DOX encapsulation }\end{array}$ & Breast cancer (in vitro \& in vivo) & Tian et al. [12] & 2014 \\
\hline & DOX-loaded tumour-cell-derived exosomes & - DOX encapsulation & Breast cancer (in vitro \& in vivo) & Yang et al. [274] & 2015 \\
\hline & Exosomes loaded with AuNPs-DOX conjugates & $\begin{array}{l}\text { - } \mathrm{pH} \text { responsive } \\
\text { - DOX conjugation }\end{array}$ & Lung cancer cell line (in vitro) & $\begin{array}{l}\text { Srivastava et al. } \\
{[275]}\end{array}$ & 2016 \\
\hline & DOX-loaded lung cell-derived nanovesicles & - DOX encapsulation & $\begin{array}{l}\text { HEK293T cells and HeLa cancer cell line } \\
\text { (in vitro) }\end{array}$ & Goh et al. [276] & 2017 \\
\hline
\end{tabular}

edges possess pH-dependent charge [262]. Like other clays, LP presents a high swelling capacity and tendency to adsorb many types of molecules at its surface. Furthermore, by reaction of the silanol groups present at its edges with alkoxysilanes with additional reactive groups, the clay can also be covalently linked to molecules of interest. Also, although this clay degrades when exposed to acidic environments, it

Table 9

DOX-based nanotherapeutics in the market and in clinical stages.

\begin{tabular}{|c|c|c|c|c|c|}
\hline Nanocarrier platform & Generic name & Formulation type & Therapeutic indication & Phase status & Refs \\
\hline \multirow[t]{21}{*}{ Liposomes } & \multirow[t]{5}{*}{ Doxil $^{\oplus} /$ Caelyx $^{\circledast}$} & \multirow[t]{5}{*}{ PEGylated liposomal DOX } & Kaposi's sarcoma & \multirow[t]{5}{*}{ Approved } & Stewart et al. [284] \\
\hline & & & Breast cancer & & Northfelt et al. [285] \\
\hline & & & Ovarian cancer & & O'Brien et al. [286] \\
\hline & & & \multirow[t]{2}{*}{ Multiple myeloma } & & Gordon et al. [287] \\
\hline & & & & & Rifkin et al. [288] \\
\hline & \multirow[t]{3}{*}{ Myocet $^{\circledast}$} & \multirow[t]{3}{*}{ Liposomal DOX } & \multirow[t]{3}{*}{ Breast cancer } & \multirow[t]{3}{*}{ Approved } & Batist et al. $[289,290]$ \\
\hline & & & & & Harris et al. [291] \\
\hline & & & & & Chan et al. [292] \\
\hline & \multirow[t]{8}{*}{ ThermoDox $^{\circledast}$} & \multirow[t]{8}{*}{ Thermosensitive liposomal DOX } & $\begin{array}{l}\text { Non-resectable hepatocellular } \\
\text { carcinoma }\end{array}$ & Phase III & NCT02112656 [293] \\
\hline & & & $\begin{array}{l}\text { Non-resectable hepatocellular } \\
\text { carcinoma }\end{array}$ & \multirow[t]{3}{*}{ Phase III } & \multirow[t]{3}{*}{ NCT00617981 [294] } \\
\hline & & & Breast cancer & & \\
\hline & & & Solid liver tumours & & \\
\hline & & & Refractory solid tumours & Phase I/II & NCT00826085 [295] \\
\hline & & & \multirow[t]{3}{*}{ Metastatic breast cancer } & Phase I & NCT02181075 [296] \\
\hline & & & & Phase I & NCT02536183 [297] \\
\hline & & & & Phase I & NCT03749850 [298] \\
\hline & Sarcodoxome $\mathrm{e}^{\mathrm{TM}}$ & Liposomal DOX containing lipochroman 6 & Small cell lung cancer & Phase II & Lopez-Pousa et al. [299] \\
\hline & \multirow[t]{2}{*}{ 2B3-101 } & \multirow[t]{2}{*}{ Glutathione PEGylated liposomal DOX } & \multirow[t]{2}{*}{ Brain metastases } & Phase II & NCT01818713 [99] \\
\hline & & & & Phase I & Kerklaan et al. [100] \\
\hline & $\begin{array}{l}\text { anti-EGFR } \\
\text { ILs-DOX }\end{array}$ & EGFR targeted liposomal DOX & Solid tumours & Phase II & Mamot et al. [101] \\
\hline & MM-302 & HER2 targeted liposomal DOX & HER2 positive breast cancer & Phase II $^{\mathrm{a}}$ & $\begin{array}{l}\text { Miller et al. }[102] \\
\text { ADCreview Website [300] }\end{array}$ \\
\hline NPs & Livatag $^{\circledast}$ & DOX-loaded poly(isohexyl-cyanoacrylate) NPs & Hepatocellular carcinoma & Phase III ${ }^{\mathrm{b}}$ & Onxeo company $[301,302]$ \\
\hline \multirow[t]{3}{*}{ Polymer-drug conjugates } & \multirow[t]{2}{*}{ FCE28068/PK1 } & \multirow{2}{*}{$\begin{array}{l}\mathrm{N} \text {-(2-Hydroxypropyl) methacrylamide-DOX } \\
\text { copolymer }\end{array}$} & Breast cancer & \multirow[t]{2}{*}{ Phase II } & \multirow[t]{2}{*}{ Seymour et al. [303] } \\
\hline & & & $\begin{array}{l}\text { Non-small cell lung cancer } \\
\text { Colorectal cancer }\end{array}$ & & \\
\hline & FCE28069/PK2 & $\begin{array}{l}\text { N-(2-Hydroxypropyl) methacrylamide-DOX- } \\
\text { Galactosamine }\end{array}$ & Primary/metastatic liver cancer & Phase II & Seymour et al. [103] \\
\hline \multirow[t]{2}{*}{ Polymeric micelles } & SP1049C & DOX block copolymer micelle & $\begin{array}{l}\text { Non-resectable stage IVb } \\
\text { adenocarcinoma }\end{array}$ & Phase III & Valle et al. [304] \\
\hline & NK911 & mPEG-DOX-poly-aspartic acid conjugates & Solid tumours & Phase II & Matsumura et al. [305] \\
\hline Bacteria-derived & $\mathrm{EDV}^{\mathrm{TM}}$ minicells & DOX-loaded EDV nanocells & Glioblastoma multiform & Phase I & Whittle et al. [306] \\
\hline
\end{tabular}

a MM-302 Phase II clinical trial was discontinued in March 2017.

b Livatag Phase III clinical trial was discontinued in September 2017. 


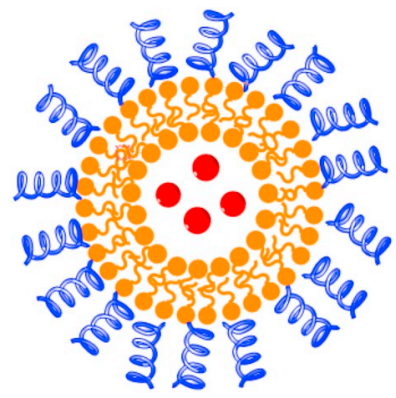

DOX
PEG
Liposome composition:
Cholesterol
HSPC
DSPE

Fig. 3. Illustration of a PEGylated Doxil ${ }^{\circledR}$ liposome.

gives rise to non-toxic products such as aqueous silica, magnesium, sodium and lithium ions [263]. For these reasons, LP is now being evaluated as a nanocarrier for diverse biological and therapeutic molecules, including for the transport of DOX. Particularly interesting, is the behaviour of LP as a "proton-sponge material" thus helping to disrupt the endolysomal compartment inside cells [32].

The first work on the use of LP for DOX delivery, was authored by Wang and colleagues [264] that used naked LP for that purpose. As can be seen in Table 7, several other LP-based nanoscale systems (nanohybrid materials) were meanwhile assayed for DOX delivery too. All these platforms were developed having in view the improvement of the behaviour of LP as a drug delivery system, namely in what concerns the drug release profiles, stability in the physiological environment and targeting purposes.

\subsection{Biological-based nanomaterials}

Over the years, the long pursuit for non-toxic, non-immunogenic and biodegradable nanomaterials also led to the possibility of considering endogenous materials as vehicles for drugs since they already make part of the physiological system. In this sense, several works arose using proteins, cells and cellular vesicles from biological origin, e.g. platelets, macrophages, erythrocytes, exosomes and albumin (from these, exosomes and albumin can be classified as nanomaterials) [266].

Examples of works using biological-based nanomaterials as carriers for DOX can be seen in Table 8. These structures were considered as potential delivery systems for DOX mainly due to their inherent role in the transport of molecules inside the human body, also associated with their high in vivo biodistribution and long-life time. For instance, human serum albumin (HSA) is one of the most abundant proteins in the human plasma. This protein has an important role in the transport of many different molecules (like hormones, fatty acids, bilirubin) and metal ions. Moreover, exosomes are membrane vesicles which occur naturally during the excretion processes of cells, and that can be found in blood and other fluids of the body. These vesicles enclose a wide variety of proteins, RNA and lipids that are mediators in cell communication.

\section{DOX-based nanotherapeutics in the clinical scenario}

As will be detailed in the following sections, several DOX-based nanotherapeutics are under clinical trials or already in clinical use. In fact, from the discovery phase and before reaching the market, drugs (and nanodrugs too) must go through a selection process that starts with preclinical studies to obtain systematic data regarding drug's pharmacodynamics (PD, what the drug does to the body) and pharmacokinetics (PK, what the body does to the drug), and consequently the determination of the PK/PD profile of the drug. One of the main objectives of the preclinical phase is to provide knowledge concerning the safety of the drug and establish the safe dose for the first-in-man study. Then, experiments in humans start step-by-step from Phase I to Phase III clinical studies. For these, specific and defined protocols should be followed that clearly establish who is qualified to participate,

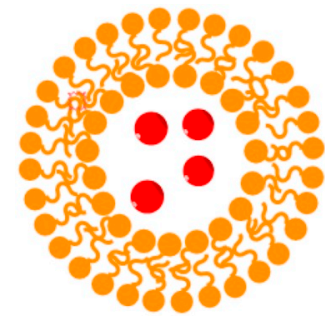

DOX

Liposome compositon: Cholesterol Egg PC

Fig. 4. Representation of the Myocet ${ }^{\circledR}$ liposome.

number of persons, study duration, administration method, dosage and how data will be collected and analysed. Still, even after the drug reaching the market, it is important to gather information from the drug's performance while it is in active medical use [277,278].

Information regarding DOX-based nanotherapeutics that where already approved for clinical use or are under clinical studies is summarized in Table 9, such as their generic name, formulation type, therapeutic indications and clinical phase status. These nanotherapeutics cover different nanoplatform types, including liposomes, nanoparticles, polymer-drug conjugates, polymeric micelles, or even biological derivatives [9,70,279-283]. It must be mentioned that Table 9 does not include generic versions or very similar variants of the listed DOX-based nanotherapeutics that meanwhile appeared in the market.

\subsection{Doxil $^{\circledast} /$ Caelyx $^{\circledast}$}

Liposomes were the first NPs to be successfully harnessed for drug delivery, and are experiencing an exponential evolution since almost 50 years ago [307]. Doxil ${ }^{\oplus}$ was the first nanotherapeutic approved by FDA in 1995 [308]. Doxil ${ }^{\oplus}$ was pioneer in the field of drug carriers in the US market and, in Europe, is commercialized under the name Caelyx ${ }^{\circledR}$ [68]. At the beginning, Doxil ${ }^{\circledast}$ was approved for the treatment of AIDS-related Kaposi's sarcoma [309] and, later on, for recurrent ovarian cancer (1998) [310], metastatic breast cancer (2003) [311] and multiple myeloma (2007) [312]. This system is based on a PEGylated liposome containing DOX in the internal cavity and has a mean diameter of about 80 to $90 \mathrm{~nm}$ [313]. Doxil ${ }^{\circledast}$ liposome is composed of three main lipid components: the hydrogenated soy phosphatidylcholine (HSPC); cholesterol and 1,2-distearoyl-sn-glycero-3-phosphoethanolamine-N-(methoxy-PEG) (DSPE-PEG) (Fig. 3). These lipids are considered safe once they do part of the diet and they are present on the cell membrane. The molar ratios (56: 38: 5) among them are responsible for maintaining the liposome structure [313,314]. The rigid bilayer at physiological temperature is achieved by the ratio between HSPC and cholesterol. DSPE is incorporated in the liposome bilayer and works as a docking point for PEG conjugation. Doxil ${ }^{\circledast}$ was conceived considering three main objectives: 1 ) to avoid the retention by the mononuclear phagocyte system and, as consequence, to prolong the circulation time; 2) to achieve a high and stable loading of DOX; and 3) to have the lipid bilayer in a "liquid order" phase. All of these requirements were achieved using: PEGylation to improve the biodistribution; the transmembrane ammonium-sulphate $\left(\left(\mathrm{NH}_{4}\right)_{2} \mathrm{SO}_{4}\right)$ gradient driven force for DOX encapsulation; and the use of HSPC which exhibits a high phase transition temperature (melting temperature, Tm). Importantly, liposomal DOX displayed linear pharmacokinetics over the dose range of 10 to $20 \mathrm{mg} / \mathrm{m}^{2}$ [313].

Interestingly, Barenholz and co-workers [313] developed a remote drug loading approach which was responsible for the high efficiency and stable drug loading. This method relies on a transmembrane gradient of $\left(\mathrm{NH}_{4}\right)_{2} \mathrm{SO}_{4}$, which involves a higher concentration of $\left(\mathrm{NH}_{4}\right)_{2} \mathrm{SO}_{4}$ inside the liposome when compared with the outside. This difference between the concentration in both compartments works as a driving force for the loading of DOX. With this loading technique, it was possible to reach a very high accumulation of DOX in the core (around 15,000 DOX molecules/liposome). 
According to literature, more than ten Phase I/II clinical trials were performed in patients suffering from AIDS-related Kaposi's sarcoma. Overall, patients treated with Doxil ${ }^{\circledR}$ formulation presented improved responses when compared with conventional chemotherapy [315]. Passing to the Phase III clinical trials, two independent randomized studies were performed [284,285]. Stewart and colleagues [284] evaluated if Doxil ${ }^{\circledR}$ could be an effective approach when compared with the traditional bleomycin and vincristine treatment. They studied 241 patients in a randomized study where $20 \mathrm{mg} / \mathrm{m}^{2}$ of Doxil ${ }^{\circledR}$ was administered against the combination of $15 \mathrm{IU} / \mathrm{m}^{2}$ bleomycin with 1.4 $\mathrm{mg} / \mathrm{m}^{2}$ vincristine. In another study performed by Northfelt et al. [285], Doxil $^{\circledR}$ efficacy was accessed versus the conventional treatment with DOX, bleomycin and vincristine. A total of 258 patients with AIDS-related Kaposi's sarcoma participated in the study. The treatment consisted in the administration of $20 \mathrm{mg} / \mathrm{m}^{2}$ Doxil $^{\circledR}$ versus the combination of $20 \mathrm{mg} / \mathrm{m}^{2}$ DOX, $10 \mathrm{mg} / \mathrm{m}^{2}$ bleomycin and $1 \mathrm{mg}$ vincristine. In both studies, Doxil ${ }^{\circledR}$ improved the treatment, being more effective and less toxic than traditional chemotherapy. Regarding ovarian cancer treatment, Gordon and colleagues [287] performed a Phase III clinical trial with the purpose of evaluating the long-term survival. The study was performed with a total of 481 patients randomly distributed in two groups. In the first group, $50 \mathrm{mg} / \mathrm{m}^{2}$ Doxil ${ }^{\circledast}$ was administered every $4^{\text {th }}$ week; in the second group, $1.5 \mathrm{mg} / \mathrm{m}^{2} /$ day of topotecan was administered during 5 days, repeating the dose at every 3 weeks. This follow-up study demonstrated that treating patients suffering from recurrent and refractory ovarian cancer with Doxil ${ }^{\circledR}$ significantly improved the overall survival (OS) (from 70.1 weeks for topotecan to 108 weeks for Doxil ${ }^{\circledR}$ patients). Therefore, these results proved that Doxil ${ }^{\circledast}$ could be used as first-line treatment for this type of cancer. Also, O'Brien et al. [286] showed that Doxil ${ }^{\circledR}$ has higher efficacy and less cardiotoxicity than conventional DOX and that could be used as first-line treatment for metastatic breast cancer. In this Phase III clinical study, around 509 women received $50 \mathrm{mg} / \mathrm{m}^{2}$ of Doxil ${ }^{\circledR}$ every 4 weeks or $60 \mathrm{mg} / \mathrm{m}^{2}$ of DOX every 3 weeks. The results showed that Doxil ${ }^{\circledR}$ led to an overall reduction in cardiotoxicity and myelosuppression, having an efficacy equivalent to that of DOX. Rifkin and co-workers [288] conducted a Phase III clinical trial in patients newly diagnosed with active multiple myeloma. The patients $(\mathrm{n}=192)$ were split and exposed to two different treatment methodologies. In the first method, the treatment consisted in the combination of $40 \mathrm{mg} / \mathrm{m}^{2}$ Doxil $^{\circledR}$ with $1.4 \mathrm{mg} / \mathrm{m}^{2}$ vincristine plus the reduction in the oral dose of dexamethasone (40 $\mathrm{mg}$ ) in the first 4 days. The second methodology was based on $0.4 \mathrm{mg} /$ day vincristine, $9 \mathrm{mg} / \mathrm{m}^{2} /$ day DOX and, also, a reduction in the dexamethasone dose for 4 days. At the end, both approaches gave a similar response, less toxicity and improved OS when compared with conventional treatment with DOX.
Doxil $^{\circledR}$ approval was the primary step to launch other nanomedicines. Following Doxil ${ }^{\circledR}$, several other lipid-based systems were created, either based on stealth liposomes with a cocktail of loaded drugs or on liposomes with loaded drugs and targeted moieties conjugated at the surface.

\subsection{Myocet $^{\circledast}$}

Five years after Doxil $^{\circledR}$ approval, Myocet ${ }^{\circledR}$, a non-PEGylated liposomal DOX, was approved in Europe and in Canada [290]. Myocet ${ }^{\circledR}$ liposome presents a diameter size around $150-250 \mathrm{~nm}$ and is composed by cholesterol, egg phosphatidylcholine (PC) and, in the interior, a DOX citrate complex (Fig. 4) [316].

This formulation was approved as first-line treatment for metastatic breast cancer in combination with cyclophosphamide [292,317]. Bearing in mind the results from preclinical stage [318], a Phase I clinical trial [319] was conducted in 38 patients with refractory solid tumours. The study was made using two different approaches. The first consisted in intravenous (i.v.) administration of a dosage of $20 \mathrm{mg} / \mathrm{m}^{2}$ escalating to $30,45,60,75$ and $90 \mathrm{mg} / \mathrm{m}^{2}$ every 3 weeks. The second involved a consecutive administration for three days, starting with 20 , then 25 and then $30 \mathrm{mg} / \mathrm{m}^{2} /$ day. The maximum-tolerated dose (MTD) was achieved by detection of leukopenia. In the first approach, the maximum dose was $90 \mathrm{mg} / \mathrm{m}^{2}$ and for the second was $25 \mathrm{mg} / \mathrm{m}^{2} /$ day. In general, Myocet ${ }^{\circledR}$ was well tolerated and revealed fewer symptoms (nausea, vomiting and stomatitis) than free DOX. Cardiotoxicity was not detected in any of the patients. Phase III clinical trials were carried on by different research groups [289,291,292]. In the first study accomplished by Batist et al. [289], the purpose was to evaluate if the combination of Myocet $^{\circledR}$ and cyclophosphamide could significantly reduce DOX cardiotoxicity and, at the same time, the improvement of the antitumor efficacy as firstline therapy for metastatic breast cancer. In this study, 297 patients received conventional DOX or Myocet ${ }^{\oplus}\left(60 \mathrm{mg} / \mathrm{m}^{2}\right.$, i.v. $)$ and, additionally, a $600 \mathrm{mg} / \mathrm{m}^{2}$ dose of cyclophosphamide every 3 weeks. Both groups revealed a similar response, but the Myocet ${ }^{\circledR}$ group demonstrated less cardiac toxicity. Another Phase III clinical trial developed by Harris et al. [291] consisted in the i.v. administration of $75 \mathrm{mg} / \mathrm{m}^{2}$ Myocet $^{\circledR}$ or DOX each $3^{\text {rd }}$ week for the treatment of metastatic breast cancer. The obtained results revealed a comparable reaction for both groups, being more satisfactory the cardiotoxicity results of the Myocet $^{\oplus}$ group. A few years later, one more Phase III clinical trial was carried on by Chan et al. [292]. In this study, they compared the combined effect of $75 \mathrm{mg} / \mathrm{m}^{2}$ Myocet $^{\circledR}$ and cyclophosphamide against $75 \mathrm{mg} / \mathrm{m}^{2}$ epirubicin and cyclophosphamide $\left(600 \mathrm{mg} / \mathrm{m}^{2}\right.$ for both approaches) as first-line treatment for metastatic
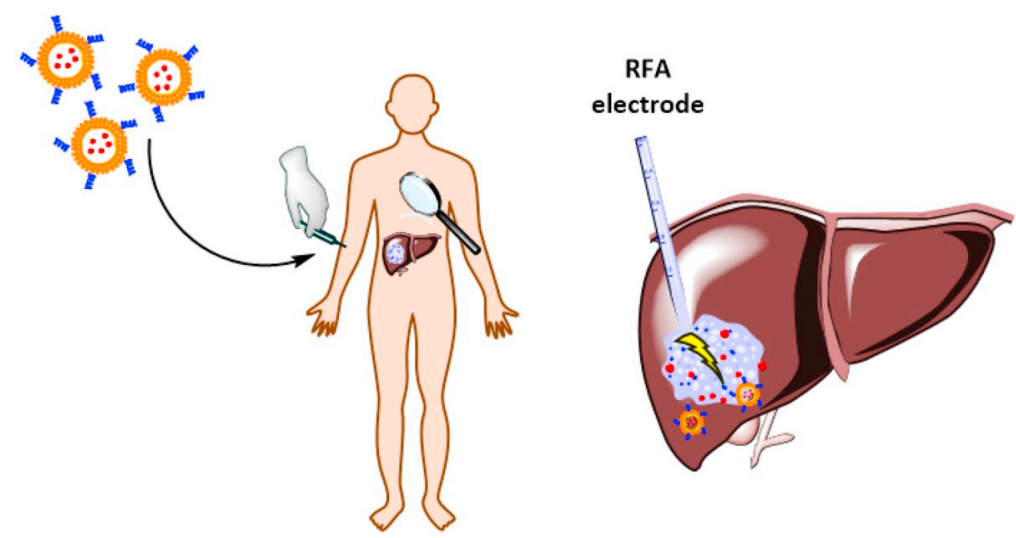

Fig. 5. Illustrative mechanism to trigger ThermoDox ${ }^{\circledast}$. 


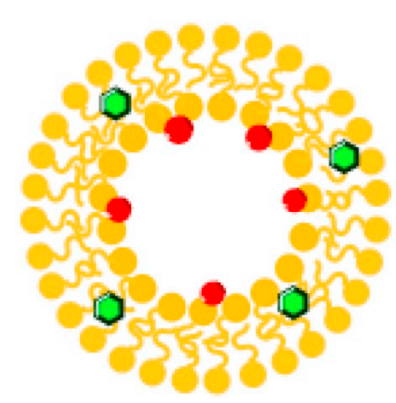

DOX

Fig. 6. Illustrative image of a liposomal formulation stabilized by lipochroman6 for DOX delivery.

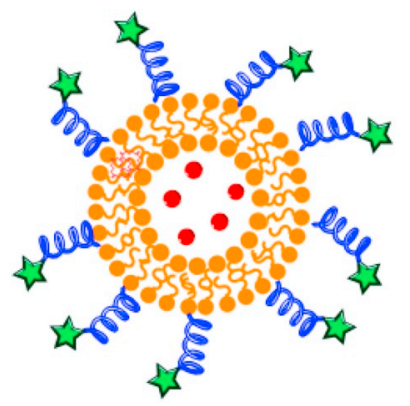

PEG DOX
Glutathione
Liposome composition:
Cholesterol
HSPC
DSPE

Fig. 7. Schematic structure of GSH-PEG liposomal DOX.

breast cancer. A total of 160 patients were randomized either to receive the first combined approach, either the second, every 3 weeks. At the end, the combination of Myocet $^{\circledR}$ and cyclophosphamide revealed to be a more promising approach as first-line treatment since it gathers the dose-effect dependability of DOX with the safety of epirubicin. All the previous clinical trials demonstrated that Myocet $^{\circledR}$ could be a good candidate for substitution of the traditional DOX.

\subsection{ThermoDox ${ }^{\circledast}$}

ThermoDox ${ }^{\circledR}$ consists of thermosensitive liposomes with DOX that have a mean diameter size of $100 \mathrm{~nm}$ [320]. The liposomes are composed of 1,2-dihexadecanoyl-sn-glycero-3-phosphocholine (DPPC), 1stearoyl-2-hydroxy-sn-glycero-3-phosphocholine (MSPC) and DSPEPEG, at molar ratios of 86: 10: 4, respectively. These systems are described as low temperature-sensitive liposomes (LTSL) since, when exposed to a relatively high temperatures $\left(\sim 42^{\circ} \mathrm{C}\right)$, become leaky and release the encapsulated drug [321,322]. The Tm of phospholipids is very important. In LTSL systems, the transition temperature of lipids is usually around 40 to $45^{\circ} \mathrm{C}$ and it is for this reason that they are used [323,324]. Phospholipids can exist in a fluid state (when the temperature is higher than their $\mathrm{Tm}$ ) or in a gel state (when the temperature is lower than their Tm). Temperature sensitive liposomes should exist in the gel state at body temperature to retain the drug while they are circulating in the bloodstream. If the temperature rises and reaches the Tm value, then the liposome changes to the fluid state and the drug is released.

ThermoDox $^{\circledast}$ formulation was conceived for the treatment of primary liver cancer (hepatocellular carcinoma, HCC) and also for recurring chest wall breast cancer. Part of the typical treatment for these pathologies is based on the use of radiofrequency ablation combined with chemotherapy $[321,325]$. In this context, ThermoDox ${ }^{\circledR}$ liposomes are delivered by i.v. administration and, due to defective vasculature, they accumulate in the tumour site. Afterwards, a source of heat is applied and in response to that stimuli, the drug is released nearby and inside of the tumour tissue (Fig. 5). The key goal of ThermoDox ${ }^{\circledast}$ is to achieve the micro-metastases which are the main responsible for cancer recurrence.

In 2009, FDA gave the status of Orphan drug (a drug developed to treat a rare medical condition) to ThermoDox ${ }^{\circledast}$ for treatment of HCC. Several clinical studies with ThermoDox ${ }^{\circledR}$ are currently ongoing, although the results have not yet been disclosed. The Phase I/II DIGNITY study (NCT00826085) [295] involved ThermoDox ${ }^{\circledR}$ and microwave hyperthermia for the treatment of breast cancer recurrence at the chest wall. Another study which is ongoing is Phase III OPTIMA study (NCT02112656) [293] which is using ThermoDox ${ }^{\circledR}$ and radiofrequency ablation (RFA) for treatment of HCC. Still another study, the Phase I HEAT study (NCT00617981) [294], started with 24 patients suffering from HCC and metastatic liver tumours. The data from this study was not published but according to Poon and Borys [320], the MTD was achieved at $50 \mathrm{mg} / \mathrm{m}^{2}$. Due to the outstanding Phase I results, this project jumped directly to Phase III. Phase III study has just been completed but the results are not yet known. The study was conducted in 701 patients aiming at treating non-resectable HCC using ThermoDox $^{\circledR}$ and RFA. If ThermoDox ${ }^{\circledR}$ and RFA have synergistic effects in the treatment, then maybe this approach can be used as front-line treatment. ThermoDox ${ }^{\circledR}$ is also being tested using different approaches. For instance, the clinical trial Phase I TARDOX (NCT02181075) [296,326] consists in a proof-of-concept study where ThermoDox ${ }^{\circledast}$ is guided by focused ultrasound (FUS) to the target and later activated by mild hyperthermia. Another study is the Phase I trial of ThermoDox ${ }^{\circledast}$ and Magnetic Resonance-Guided High Intensity Focused Ultrasound (MR-HIFU) for the treatment of relapsed or refractory solid tumours in

A

B

\section{MM-302}

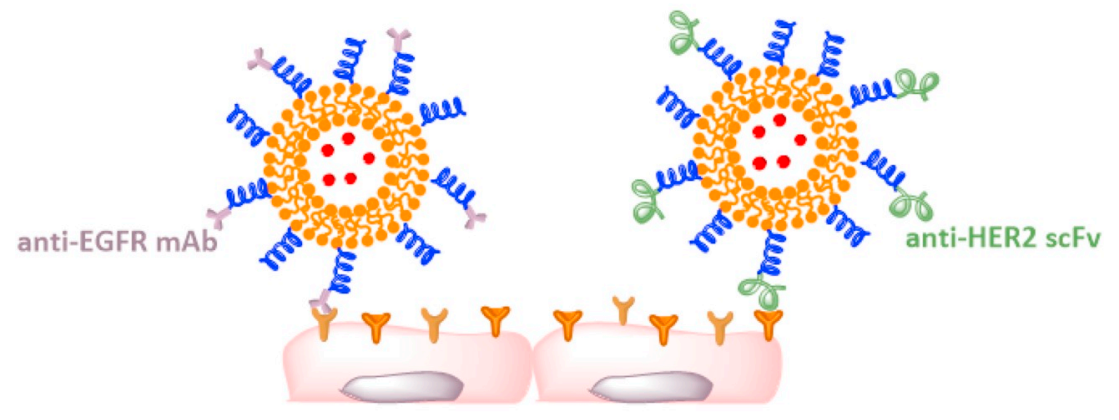

PEG-DSPE DOX Liposome compositon: Cholesterol

$$
\text { Egg PC }
$$

Fig. 8. A) Scheme showing the interaction of anti-EGFR ILs-DOX with cells; B) Illustration of the interaction of MM-302 with HER2 overexpressing cells. 
children, adolescents and young adults (NCT02536183) [297]. This study started with 34 patients and is seeking for the MTD and the recommended Phase II dose of ThermoDox ${ }^{\circledR}$ to be administered in combination with MR-HIFU. The most recent study is the Phase I trial (NCT03749850) [298] of image-guided targeted DOX delivery with hyperthermia to optimize loco-regional control in breast cancer. In this study, clinicians are interested in the evaluation of the synergistic effect of ThermoDox ${ }^{\circledast}$ with local hyperthermia and cyclophosphamide in the treatment of primary breast tumour in patients with metastatic breast cancer.

\subsection{Sarcodoxome ${ }^{\mathrm{TM}}$}

A new liposomal formulation containing DOX, Sarcodoxome ${ }^{\mathrm{TM}}$, was developed for the treatment of soft tissue sarcoma (STS). These liposomes are not PEGylated and contain lipochroman- 6 to improve their stability. In this system, DOX is loaded in the walls of the liposome (Fig. 6). In 2006, Sarcodoxome ${ }^{\mathrm{TM}}$ received the Orphan drug status by EMA and later the same status was approved by FDA (2007). Phase I/II clinical trials were launched in Spain [327]. A Phase II clinical trial [299] was performed in 37 patients with advanced or metastatic STS and with 65 years or older. In general, Sarcodoxome ${ }^{\mathrm{TM}}$ revealed a safe and acceptable toxicity profile, an MTD of $80 \mathrm{mg} / \mathrm{m}^{2}$ and no cardiotoxicity associated. However, further studies are needed with younger patients.

\section{5. $2 B 3-101$}

Brain tumours are considered devastating diseases, only starting to reveal symptoms already at a late stage. As mentioned before, the BBB is a protective shield of the central nervous system (CNS), being responsible for blocking the passage into the CNS of strange and potentially harmful molecules. As a consequence, treating brain malignancies is a very big challenge [87]. Aiming at overcoming this issue, the 2B3101 system is being developed which consists of a PEGylated liposomal DOX formulation conjugated to glutathione (GSH) as targeting ligand. It has an average diameter of $95 \mathrm{~nm}$ (Fig. 7) [86,328]. The technology behind 2B3-101 (G-technology ${ }^{\circledR}$ ) explores existing GSH-transport mechanisms across the BBB [86]. This system was designed for targeting glioma brain and metastases.

The performance (affinity and uptake) of 2B3-101 was evaluated in vitro using human brain capillary endothelial cells [328]. Results suggested that the uptake of 2B3-101 is time-, temperature- and concentration-dependent. Overall, the presence of GSH really improved the efficacy of the 2B3-101 system, increasing the efficacy of DOX delivery. Further PK and brain uptake studies were performed in vivo with concentrations comparable to those tested in vitro. Moreover, the efficacy of 2B3-101 was studied in vivo in mice using a brain tumour model of glioblastoma multiform (GBM). Basically, U87MG cells (human glioblastoma cell line) were injected directly into the brain of athymic FVB mice (mice lacking the thymus gland), originating a high vascularized brain tumour. In this study, the efficacy of free DOX, PEGylated liposomal DOX and 2B3-101 was compared. At the end, no neurological indicators were seen, and both systems were well tolerated. However, the presence of GSH in 2B3-101 resulted in a superior efficacy. The aim of this study was not to determine the toxicity and, thus, no MTD was determined [328]. However, these promising results were the initial impulse for the beginning of the clinical trials. A Phase I/IIa clinical trial [100] was performed in patients with solid tumours and brain metastases or recurrent malignant glioma. The patients received 40-70 $\mathrm{mg} / \mathrm{m}^{2}$ or $60 \mathrm{mg} / \mathrm{m}^{2}$ dosages. In general, $2 \mathrm{~B} 3-101$ was considered safe and it was well tolerated. In Phase IIa trial, the recommended doses were based on the tolerability of the previous results. The 2B3-101 system is also being studied for the treatment of meningeal carcinomatosis (NCT01818713) [99]. In the Phase II clinical trial, the aim is to evaluate the primary efficacy of 2B3-101 in patients suffering from

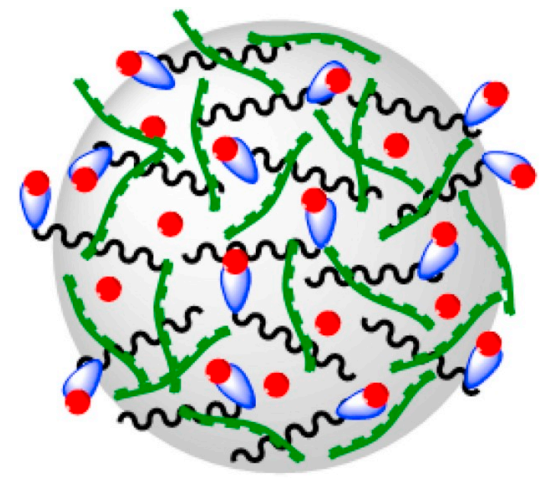

DOX

\section{PIHCA}

\section{Surfactant}

\section{Cyclodextrin}

Fig. 9. Representative scheme of PIHCA NPs loaded with DOX.

leptomeningeal metastases of breast cancer. According to the clinical trials website, just a few patients received the treatment $(n=6)$. A single dose of $50 \mathrm{mg} / \mathrm{m}^{2}$ was administered intravenously every 3 weeks. Up to date, no results were published.

\subsection{Anti-EGFR immunoliposomes-DOX}

The conjugation of monoclonal antibody fragments (mAb) to liposomes results in immunoliposomes (ILs). These anti-EGFR immunoliposomes-DOX, now in phase II clinical trial, are based on liposomes made of cholesterol and PC conjugated to a mAb against the epidermal growth factor receptor (EGFR) (Fig. 8 A) [329]. These liposomes present an average diameter of 100-120 nm [330]. They can target the EGFR overexpressing tumours and, at the same time, they can be used as drug carriers [331].

Mamot et al. studied the delivery of DOX from the anti-EGFR ILsDOX system. According to these in vitro studies [331], a higher DOX internalization (about 29- fold more) was achieved in the presence of the EGFR ligand in EGFR-overexpressing cell lines (MDA-MB-468 human breast cancer and U87MG human GBM). In the in vivo studies [332], healthy rats revealed similar PK profiles between the liposomal DOX with and without EGFR ligand, suggesting that the mAb fragment was not crucial for biodistribution stability. The therapeutic efficacy of the anti-EGFR ILs-DOX was evaluated by using the cancer cell lines previously used, but this time as xenograft models. The results clearly showed that anti-EGFR ILs-DOX could significantly inhibit the tumour size and overcome the problem of multidrug resistance [333]. Considering the positive results, this anti-EGFR ILs-DOX system proceeded for Phase I clinical trial [101]. The main goal of this study was to determine the MTD in patients with EGFR-overexpressing advanced solid tumours. In this trial, 26 patients were treated with i.v. administration of anti-EGFR ILs-DOX. The concentration was scaled up $\left(5-60 \mathrm{mg} / \mathrm{m}^{2}\right.$ DOX equivalents) over the six cycles. Interestingly, was the absence of cardiotoxicity, cumulative toxicity or alopecia. The suggested antiEGFR ILs-DOX concentration for Phase II clinical trial was $50 \mathrm{mg} / \mathrm{m}^{2}$, which corresponds to the MTD.

\section{7. $M M-302$}

A new drug delivery system, MM-302, was also developed that displays an average size of 75-110 $\mathrm{nm}$ and is a HER2-targeted antibodyliposomal DOX conjugate. HER2 is the human epidermal growth factor receptor-2 that may be overexpressed in breast cancer (Fig. 8 B) [334]. The targeting is accomplished by attaching a single-chain antibody fragment (scFv) of HER2 via a polyethylene glycol spacer (PEG-DSPE) to the DOX-loaded ILs surface [329].

In vitro studies revealed that the MM-302 liposomes were bind and internalized by HER2 overexpressing cells in a greater extent than the control $[335,336]$. According to the PK results in rats, there was no difference between the clearance rates of MM-302 and the control. 
A<smiles>CNC(=O)C(C)(C)C(C)(C)CC(=O)NCC(C)O</smiles>

B<smiles>CCC(C)(C)C(=O)NCC(C)O</smiles><smiles>CCCCCNC(=O)C[C@H](CC(=O)O)NC(=O)[C@@H](N)Cc1cccc(C(C)C)c1</smiles>

Polymer

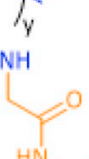<smiles>C[C@H]1O[C@H](O)C[C@H](NC=O)[C@H]1O</smiles>

Degradable peptide<smiles>CCO[C@H]1C[C@@](O)(C(=O)CO)Cc2c(O)c3c(c(O)c21)C(=O)c1c(OC)cccc1C3=O</smiles><smiles>CNCC(=O)N[C@H]1C[C@H](O)O[C@@H](O)[C@H]1O</smiles>

Galactosamine

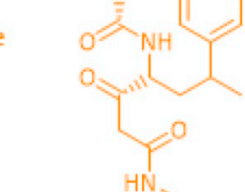<smiles>C1CC2CC12</smiles>

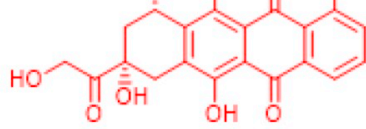

Doxorubicin
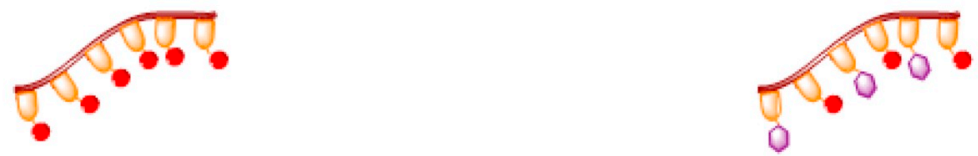

Fig. 10. A) HPMA copolymer-DOX (PK1) structure; B) HPMA copolymer-DOX structure containing galactosamine (PK2) to promote liver targeting.

Furthermore, the targeting capacity of MM-302 liposomes was extensively studied in four different human HER2-positive breast cancer xenograft models. These studies confirmed the selectivity for HER2 positive cells and, as a result, in some cases, a significant decrease in tumour growth $[335,336]$. According to Phase I trial results reported at the San Antonio Breast Cancer Symposium in 2012 [337], it was found that MTD was $40 \mathrm{mg} / \mathrm{m}^{2}$. In this trial, 14 patients with positive HER2 advanced breast cancer received the MM-302. The administered dosage was $8,16,30$ and $40 \mathrm{mg} / \mathrm{m}^{2}$ every 4 weeks. In general, the results obtained suggested no cardiotoxicity associated up to the maximum dose administered.

Phase II clinical trial (HERMIONE study) consisted in the random administration of MM-302 plus trastuzumab in patients with locally advanced/metastatic HER2-positive breast cancer [102]. The main key points of this study were to assess the progression-free survival (PFS), the OS and also the safety, tolerability, quality of life and PK profile. For MM-302, the selected dose was $30 \mathrm{mg} / \mathrm{m}^{2}$ every 3 weeks, and the combination with trastuzumab was also administered every 3 weeks. At the end, results demonstrated that the combination of both novel MM302 and trastuzumab therapy could be well-tolerated and more effective. Unfortunately, after a recommendation of the independent monitoring panel "Data and Safety Monitoring Board" (DSMB), the HERMIONE clinical trial was stopped. The decision was taken considering that there were no improved safety signals after the treatment.

\subsection{Livatag $^{\circledast}$}

Livatag $^{\circledR}$ is a NP formulation of DOX (Transdrug ${ }^{\mathrm{TM}}$ technology) that consists in DOX-loaded polyisohexylcyanoacrylate (PIHCA) NPs with $300 \mathrm{~nm}$ size (Fig. 9) [338]. The Transdrug ${ }^{\mathrm{TM}}$ technology relies on the use of NPs to overcome drug resistance, facilitating cell penetration and cell-drug contact.

Livatag ${ }^{\circledR}$ received the status of Orphan drug in Europe in 2004 and in the US in 2011. This NP formulation was developed with the aim to treat patients with primary liver cancer [339]. In Phase I clinical trial (ReLive study), Kattan and colleagues [338] have studied the effect of Livatag ${ }^{\circledR}$ in patients with refractory solid tumours. A total of 21 patients have received the formulation by i.v. administration with an initial dose of $15 \mathrm{mg} / \mathrm{m}^{2}\left(30,45,60,75\right.$ and $\left.90 \mathrm{mg} / \mathrm{m}^{2}\right)$ every 4 weeks. The MTD revealed neutropenia at $90 \mathrm{mg} / \mathrm{m}^{2}$. Consequently, for further Phase II trial, it was suggested a dose of $75 \mathrm{mg} / \mathrm{m}^{2}$. According to the Onxeo website [340], the results from Phase II showed an increase in the survival time of patients with HCC. After that, a Phase III clinical trial was launched in 2012, in the US and Europe. This trial was designed to study the efficacy of Livatag ${ }^{\circledR}$ in 400 patients with HCC at advanced stage. At an early stage, Phase III results exhibited good results and tolerance. Unfortunately, in September 11, 2017, it was announced that the ReLive study did not met the principal purposes which were to improve the overall patient's survival when compared to the control group [301]. The final results from ReLive study were presented at the $11^{\text {th }}$ Annual Conference of the International Liver Cancer Association in Seoul, South Korea (ILCA 2017) [302].

\section{9. $P K 1$}

Currently, few anticancer-drug conjugates achieved the clinical phase. A few years back, Kopeček [341] and co-workers started the investigation on (N-(2-hydroxypropyl) methacrylamide) (HPMA) synthesis. Later on, fruit of collaborations with Duncan et al., a patent application arise (1985) [342]. Until now, two types of HPMA copolymer conjugates were developed and reached the clinical trial stage. PK1 was the first to be designed and consists in a HPMA backbone in which DOX is conjugated through a peptide linker (Gly-Phe-Leu-Gly) (Fig. $10 \mathrm{~A}$ ). This linker is stable at physiological pH but can be cleaved in the lysosomes by enzymes. PK1 presents a molecular weight (MW) $30,000 \mathrm{~g} / \mathrm{mol}$ and DOX content around $8.5 \mathrm{wt} \%$ [343]. At preclinical stage [344], this nanotherapeutic revealed to be promising when compared to the conventional drug. In a Phase I clinical trial [343], PK1 


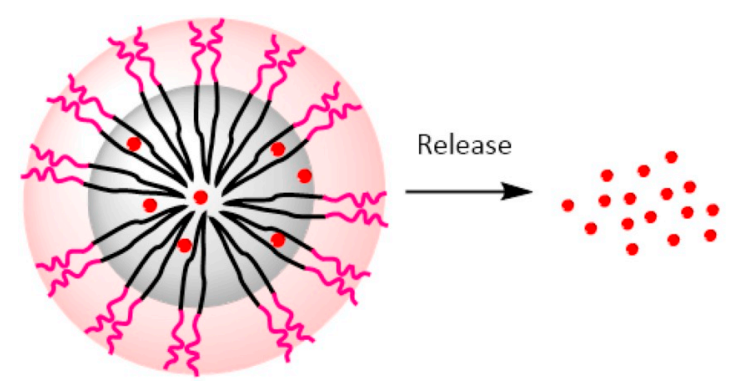

DOX

Fig. 11. Schematic representation of drug-loaded polymeric micelle.

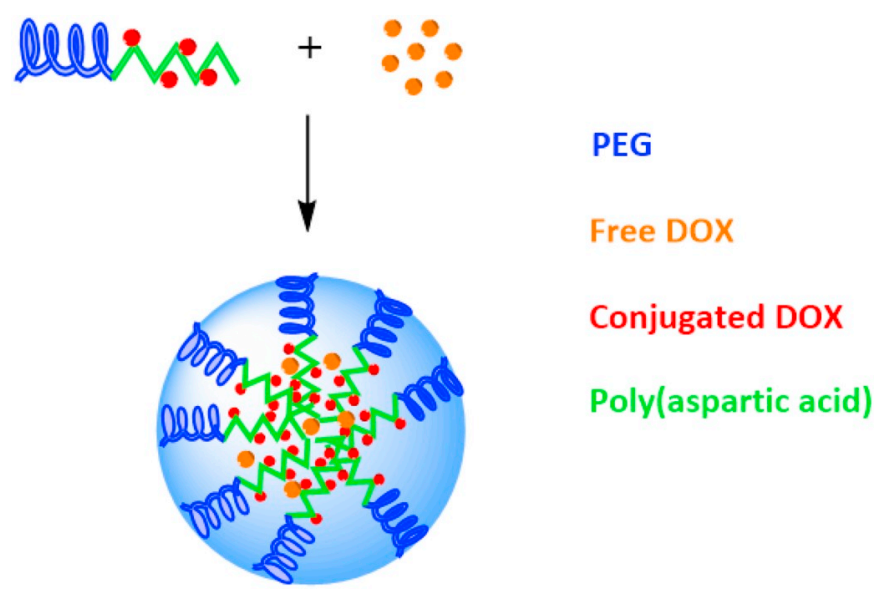

Fig. 12. Schematic representation showing the structure of NK911.

was administered to 36 patients with refractory or resistant cancer by i.v. administration with an interval of 3 weeks between cycles. The object of study was to determine the PK profile of PK1 and the toxicity associated with the determination of the MTD and dose-limiting toxicities (DLTs). At the beginning of the treatment, $20 \mathrm{mg} / \mathrm{m}^{2}$ of PK1 were administered and increased until reaching $320 \mathrm{mg} / \mathrm{m}^{2}$. At this step, few toxic effects were registered, namely mucositis and febrile neutropenia. Interestingly is that no cardiotoxicity was observed even at $1680 \mathrm{mg}$ / $\mathrm{m}^{2}$. Based on these results, the recommended dose to Phase II was 280 $\mathrm{mg} / \mathrm{m}^{2}$, to be implemented in patients with colorectal, non-small cell lung cancer (NSCLC) and breast cancer patients. In Phase II clinical trials [303], this recommendation was considered and 62 patients were divided into the three cancer types: breast $(n=17)$, NSCLC $(n=29)$ and colorectal $(n=16)$ cancer. The response was favourable in few cases ( 3 for breast and 3 for NSCLC) and no response for colorectal patients. In contrast, these studies demonstrated that the administration of high doses of PK1 $\left(>20 \mathrm{~g} / \mathrm{m}^{2}\right)$ did not triggered any toxicity related to the polymer or even immunogenicity.

\subsection{0. $P K 2$}

Bearing in mind the PK1 system and the positive results achieved, PK2 was developed, being structurally similar to PK1 but with an additional galactosamine residue (Fig. $10 \mathrm{~B}$ ). This residue introduction is supposed to facilitate and improve the efficacy of the system by targeting the hepatocyte asialoglycoprotein receptors for hepatic cancer treatment. The MW of PK2 is about $25,000 \mathrm{~g} / \mathrm{mol}$ with a DOX content of $\sim 7.5 \%$ and $1.5-2.5 \mathrm{~mol} \%$ of galactosamine content [103]. PK2 is the first drug conjugate which was designed for active targeting. In preclinical studies with mice, reduced cardiotoxicity was observed when using PK2 [345]. In a Phase I study [103], the PK profile, toxicity and the targeting specificity were evaluated in 31 patients with primary or metastatic liver cancer. PK2 was administered by i.v. with an initial concentration of $20 \mathrm{mg} / \mathrm{m}^{2}$ (DOX equivalents) every 3 weeks. Consequently, with the escalation of the concentration $\left(160 \mathrm{mg} / \mathrm{m}^{2}\right.$, further MTD), some side effects started to appear, such as severe fatigue, neutropenia and mucositis. Moreover, after $24 \mathrm{~h}$ injection, the biodistribution revealed that approximately $16.9 \%$ of the PK2 drug was targeting the liver, while the untargeted control did not. For further Phase II trials, a $120 \mathrm{mg} / \mathrm{m}^{2}$ dosage was recommended to be administered every 3 weeks.

\subsection{1. $S P 1049 C$}

SP1049C consists in micelles resulting from the combination of two different Pluronic ${ }^{\circledR}$ copolymers, i.e., Pluronic ${ }^{\circledR}$ L61 and Pluronic ${ }^{\circledR}$ F127 [346]. Basically, pluronics consist in ternary copolymers of poly(ethylene oxide) (PEO) and poly(propylene oxide) (PPO). Each of these

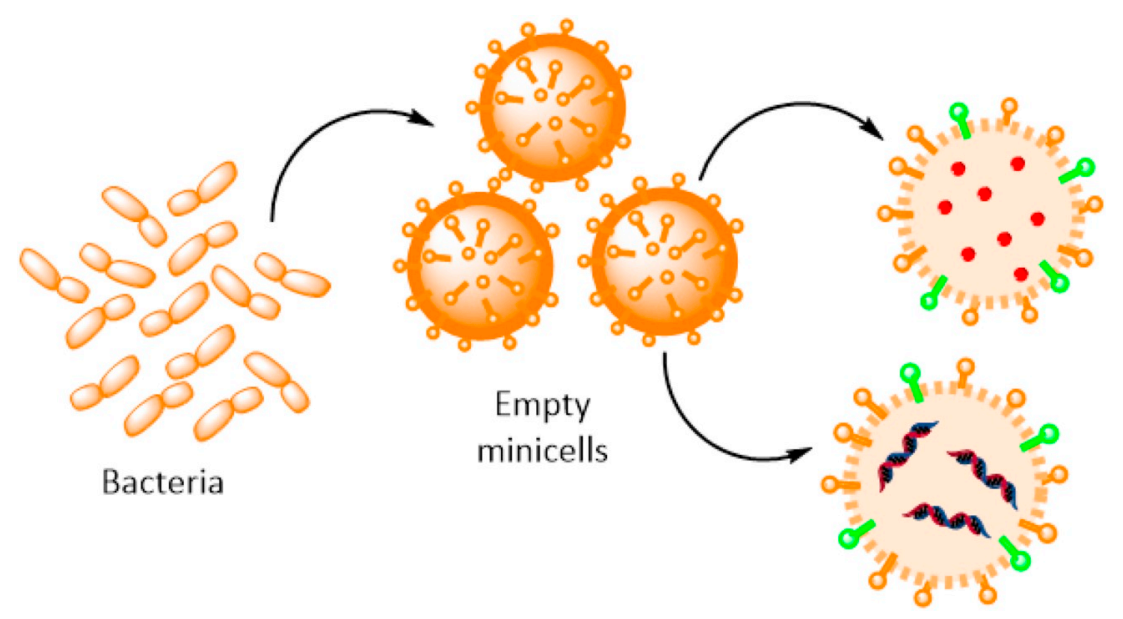

Fig. 13. Scheme showing bispecific antibody-targeted, drug/siRNA-packaged minicells. 
segments is responsible for one part of the micelle formation. The PPO segment is hydrophobic and will assemble forming the hydrophobic core, whereas the PEO segment is hydrophilic and will be responsible for the corona formation (Fig. 11) [347]. The ratio Pluronic ${ }^{\oplus}$ L61:Pluronic ${ }^{\circledast}$ F127 used to obtain the desired polymeric micelle was 1:8 (w/w). Afterwards, DOX was loaded into the hydrophobic core by noncovalent interactions achieving a diameter of 22-27 nm [346].

In vitro assays demonstrated that SP1049C had an improved efficacy when compared to free DOX [348]. Furthermore, the preclinical in vivo studies showed that the antitumor efficacy improved with SP1049C usage [346,349]. SP1049C had antitumor potential especially for treating adenocarcinoma in the oesophagus and gastroesophageal junction. In Phase I clinical trials [350], the goal was to assess all the PK and toxicity profiles, specifically, the DLTs and MTD. The study started with 28 patients with refractory tumours and a $5 \mathrm{mg} / \mathrm{m}^{2}$ (DOX equivalents) dose every 3 weeks till reaching the $6^{\text {th }}$ cycle. When the maximum dose was administered $\left(90 \mathrm{mg} / \mathrm{m}^{2}\right)$, some toxic effects were observed, such as myelosuppression. Considering these results, a Phase II clinical trial was proposed but with a DLT around $70 \mathrm{mg} / \mathrm{m}^{2}$. The Phase II clinical trial [304] included 21 patients with adenocarcinoma in the oesophagus and gastroesophageal junction. In this study, a 75 $\mathrm{mg} / \mathrm{m}^{2}$ (DOX equivalents) dose was injected every 3 weeks. Despite neutropenia manifestation, this Phase II revealed that SP1049C was really effective as monotherapy for the previously mentioned types of cancer. A Phase III clinical trial is currently under way for metastatic adenocarcinoma of the oesophagus, gastroesophageal junction and stomach. In the meantime, FDA approved SP1049C as an orphan drug for gastric cancer.

\subsection{NK911}

The NK911 is also a polymeric micellar formulation of DOX. This system is made of a copolymer of PEG (MW $\sim 5,000 \mathrm{~g} / \mathrm{mol}$ ) and polyaspartic acid (ASP) (Fig. 12). To achieve a higher hydrophobicity, DOX was partially conjugated in the side chains of ASP $(-45 \%)$. Therefore, when the copolymer is dissolved in water, it assembles as a micelle with a high hydrophobic inner core. The hydrophobicity of the core provides additional accommodation to encapsulate free DOX. As a result, the DOX which will be responsible for the antitumor activity is the loaded one, since the conjugated one does not reveal any activity. This lack of response is probably due to the stable coupling of DOX to the backbone of the polymer. NK911 exhibits a small size, nearby 40 $\mathrm{nm}$ in diameter, which is within the NPs size for passive targeting by the EPR effect [351].

This NK911 system successfully accumulated in solid tumours in mice and was thus considered for Phase I clinical trial. In this study, a total of 23 patients with metastatic or recurrent solid tumours were followed. The aim was to analyse the PK profile of NK911 nanotherapeutics through the MTD and the DLTs. The treatment consisted in i.v. administration of the NK911 formulation, starting with $6 \mathrm{mg} / \mathrm{m}^{2}$ DOX equivalent every 3 weeks. The haematological side effect most common was neutropenia when the doses were increased till 50 to $67 \mathrm{mg} / \mathrm{m}^{2}$ DOX equivalents. Other associated effects were mild alopecia, anorexia and stomatitis. In general, NK911 was well tolerated and presented a good safety profile. A Phase II clinical trial was proposed with a recommended dosage of $50 \mathrm{mg} / \mathrm{m}^{2}$ every 3 weeks, however, it is uncertain if the clinical trials proceeded [305].

\subsection{Bacterial-derived $E D V^{\mathrm{TM}}$ minicells}

MacDiarmid and colleagues [352] accomplished a novel technology based on a bacterial-derived nanoplatform (EDV ${ }^{\mathrm{TM}}$ minicells) for drug/ gene encapsulation with specific targeting ability (Fig. 13). These systems are obtained through a genetically minCDE-chromosomal deletion mutant from: Salmonella enterica serovar Typhimurium (S. Typhimurium); Escherichia coli; Shigella flexneri; Pseudomonas aeruginosa
(Gram-negative) and Listeria monocytes (Gram-positive) strains. Essentially, bacterial minicells are anucleate NPs that present a uniform diameter $(\sim 400 \mathrm{~nm})$, acquired by the inactivation of the genes that control normal bacterial cell division, therefore depressing the polar sites of cell fission. They are produced with high yields from both Grampositive and Gram-negative bacteria. After the production and purification process, the bacterial minicells can be lyophilized and stored for about 4 months. They can be used as vectors for a wide range of chemotherapeutics with different charge, structure, solubility and hydrophobicity. The encapsulation process occurs by unilateral diffusion and shows to be dependent on concentration and time of incubation with the drug.

The targetability of these systems is accomplished by using bispecific antibodies, in which one arm will recognize the surface lipopolysaccharide, and the other will recognize a cell-surface receptor specific for the targeted cell, such as EGFR [353]. A single minicell can accommodate approximately 1 million molecules of DOX [352]. Once in the tumour microenvironment, the endocytosis process is triggered by the binding of the targeted-minicell to the specific antibody receptor present on the tumour cell surface. According to the in vitro studies (MDA-MB-468 breast, SKOV-3 ovarian, A549 lung, and HL-60 promyelocytic leukaemia cancer cell lines), minicells are internalized and degraded by the endosomes/lysosomes and, as a result, the cargo is released into the cytosol [352,354]. In vivo studies were performed with targeted DOX-loaded bacterial minicells to evaluate the antitumor potential. These experiments resulted in a huge inhibition and regression of the tumour growth either for mice with cancer xenografts (breast, lung, ovarian and breast) and for dogs with Non-Hodgkin's lymphoma (NHL) [352]. Besides cancer models, healthy pigs were also used to evaluate the safety of the i.v. administration of bacterial minicells. Despite the five consecutives i.v. administrations, pigs tolerate well and did not reveal side effects for all haematological indices, serum chemistries, growth and food intake. The same was verified for the NHL dogs. Furthermore, there is the need to highlight that more exhaustive toxicology and stability studies are necessary for using this bacterial minicell in humans. The previous in vitro and in vivo results were responsible for the achievement of the "first-in-man" clinical trial. This study was based on EDV with the anti-human EGFR Erbitux and paclitaxel ( ${ }^{\text {Erbitux }} \mathrm{EDV}_{\text {Paclitaxel }}$ ) [355]. Another Phase I clinical trial which is currently under progress is the CerebralEDV study (NCT02766699) [306]. The purpose of this research is to study the safety and tolerability of the EDV minicell (EGFR(V)-EDV-DOX $\left({ }^{\mathrm{V}} \mathrm{EDV}_{\mathrm{DOX}}\right)$ ) packaged with DOX and coupled to panitumumab/Vectibix (V) to target the EGFR protein on the tumour cell membrane. The choice of the EGFR as target moiety and the Vectibix as the antibody was based on literature, where EGFR seems to be important for GBM [356,357]. In this study, the patients with recurrent or progressive GBM randomly received one of two ${ }^{\mathrm{E}} \mathrm{EDV}_{\mathrm{DOX}}$ doses $\left(5 \times 10^{9}\right.$ or $\left.8 \times 10^{9}\right)$ by i.v. administration, once a week, for a period of 8 weeks. In general, ${ }^{\mathrm{EDVV}_{\text {DOx }}}$ was well tolerated and no severe side effects were reported, being the most common, fever, nausea and chills. However, MTD was not achieved. In summary, this Phase I trial revealed that ${ }^{{ }^{2}} \mathrm{EDV}_{\mathrm{DOx}}$ can be administered to the patients with no severe risks [306]. Nevertheless, further research is needed to validate the safety of this novel technology. Meanwhile, in 2017, FDA approved the ${ }^{\mathrm{EGFR}} \mathrm{EDV}_{\text {DOX }}$ minicells as an orphan drug status for the treatment of GBM.

\section{Conclusions and future perspectives}

Nanotechnology, being a multi and interdisciplinary field, offers new opportunities for patient treatment. In the context of cancer, the introduction of nanomaterials as nanocarriers for conventional drugs is extending the possibility of their use, by improving their efficacy and safety. This is the case of DOX, an anthracycline widely applied in cancer treatment which has been associated to the occurrence of severe side effects. Although there is a long road to pursue until a 
nanotherapeutic reaches the market, a few DOX-based nanotherapeutics are now in the clinical scenario and others are currently under different phases of clinical trials. While liposomes are clearly ahead in the field of DOX-based nanotherapeutics, other nanoscale formulations are also now showing their applicability and specific advantages, such as nanoparticles, polymer-drug conjugates, micelles and nanocapsules from biological origin. Interestingly, one can notice that these DOXnanotherapeutics are evolving, not only exploring the EPR effect to accumulate and exert their action in the tumour site, but they are getting smarter over time and equipped with new tools that allow them to overcome physiological barriers, respond to environmental stimuli and reach specific cells/molecular targets.

Meanwhile, research on the area of DOX-based nanotherapeutics is still very active and results are exciting. Given the number of publications that can be found in the literature, of which only representative examples are presented in this review, new and better solutions for the delivery of doxorubicin in cancer cells may be expected in the future, which will also possible be extended for the delivery of other drugs. Hopefully, in a medium/long-term, the future of cancer therapy will rely on personalized nanomedicine approaches, custom-made for each patient, and capable of treating not only primary tumours but also their metastases.

\section{Acknowledgments}

Fundação para a Ciência e Tecnologia (FCT), I.P. is acknowledged for the Ph.D. scholarship of M.G. (SFRH/BD/88721/2012) and the CQM Strategic Project UID/QUI/00674/2019 (Portuguese Government Funds). The project Centro de Química da Madeira - CQM ${ }^{+}$(M142001-0145-FEDER-000005, ARDITI) funded by Madeira Regional Operational Programme (Madeira 14-20) is also acknowledged.

\section{References}

[1] International Agency for Research on Cancer, World Health Organization - Cancer fact sheets, http://gco.iarc.fr/today, (2018).

[2] National Cancer Institute, What is cancer? Natl. Cancer Inst. (2015), https://www. cancer.gov/about-cancer/understanding/what-is-cancer , Accessed date: 17 June 2019.

[3] C. Guozhong, A. Müller, A.K. Cheetham, Nanomaterials - an introduction, Nanostructures Nanomater. Synth. Prop. Appl., World scientific, 2004, pp. 1-11.

[4] J.L. Markman, A. Rekechenetskiy, E. Holler, J.Y. Ljubimova, Nanomedicine therapeutic approaches to overcome cancer drug resistance, Adv. Drug Deliv. Rev. 65 (2013) 1866-1879, https://doi.org/10.1016/j.addr.2013.09.019.

[5] R.A. Freitas, What is nanomedicine? Nanomedicine 1 (2005) 2-9, https://doi.org/ 10.1016/j.disamonth.2005.08.003.

[6] J. Shi, P.W. Kantoff, R. Wooster, O.C. Farokhzad, Cancer nanomedicine: progress, challenges and opportunities, Nat. Rev. Cancer 17 (2017) 20-37, https://doi.org/ 10.1038/nrc. 2016.108.

[7] P.T. Wong, S.K. Choi, Mechanisms of drug release in nanotherapeutic delivery systems, Chem. Rev. 115 (2015) 3388-3432, https://doi.org/10.1021/cr5004634.

[8] G. Chen, I. Roy, C. Yang, P.N. Prasad, Nanochemistry and nanomedicine for nanoparticle-based diagnostics and therapy, Chem. Rev. 116 (2016) 2826-2885, https://doi.org/10.1021/acs.chemrev.5b00148.

[9] J.I. Hare, T. Lammers, M.B. Ashford, S. Puri, G. Storm, S.T. Barry, Challenges and strategies in anti-cancer nanomedicine development: an industry perspective, Adv. Drug Deliv. Rev. 108 (2017) 25-38, https://doi.org/10.1016/j.addr.2016.04.025.

[10] N. Bertrand, J. Wu, X. Xu, N. Kamaly, O.C. Farokhzad, Cancer nanotechnology: the impact of passive and active targeting in the era of modern cancer biology, Adv. Drug Deliv. Rev. 66 (2014) 2-25, https://doi.org/10.1016/j.addr.2013.11.009.

[11] I.-H. Lee, S. An, M.K. Yu, H.-K. Kwon, S.-H. Im, S. Jon, Targeted chemoimmunotherapy using drug-loaded aptamer-dendrimer bioconjugates, J. Control. Release 155 (2011) 435-441, https://doi.org/10.1016/j.jconrel.2011.05.025.

[12] Y. Tian, S. Li, J. Song, T. Ji, M. Zhu, G.J. Anderson, J. Wei, G. Nie, A doxorubicin delivery platform using engineered natural membrane vesicle exosomes for targeted tumor therapy, Biomaterials. 35 (2014) 2383-2390, https://doi.org/10. 1016/j.biomaterials.2013.11.083.

[13] M. Fan, X. Liang, D. Yang, X. Pan, Z. Li, H. Wang, B. Shi, Epidermal growth factor receptor-targeted peptide conjugated phospholipid micelles for doxorubicin delivery, J. Drug Target. 24 (2016) 111-119, https://doi.org/10.3109/1061186X. 2015.1058800

[14] Y. Zhu, J. Zhang, F. Meng, C. Deng, R. Cheng, J. Feijen, Z. Zhong, cRGD-functionalized reduction-sensitive shell-sheddable biodegradable micelles mediate enhanced doxorubicin delivery to human glioma xenografts in vivo, J. Control. Release 233 (2016) 29-38, https://doi.org/10.1016/j.jconrel.2016.05.014.
[15] H. Wu, H. Jin, C. Wang, Z. Zhang, H. Ruan, L. Sun, C. Yang, Y. Li, W. Qin, C. Wang, Synergistic Cisplatin/Doxorubicin Combination Chemotherapy for MultidrugResistant Cancer via Polymeric Nanogels Targeting Delivery, ACS Appl. Mater. Interfaces 9 (2017) 9426-9436, https://doi.org/10.1021/acsami.6b16844.

[16] Y. Fang, W. Yang, L. Cheng, F. Meng, J. Zhang, Z. Zhong, EGFR-targeted multifunctional polymersomal doxorubicin induces selective and potent suppression of orthotopic human liver cancer in vivo, Acta Biomater. 64 (2017) 323-333, https://doi.org/10.1016/j.actbio.2017.10.013.

[17] Y. Zhuang, L. Zhao, L. Zheng, Y. Hu, L. Ding, X. Li, C. Liu, J. Zhao, X. Shi, R. Guo, A. LAPONITE-Polyethylenimine, Based theranostic nanoplatform for TumorTargeting CT Imaging and Chemotherapy, ACS Biomater. Sci. Eng. 3 (2017) 431-442, https://doi.org/10.1021/acsbiomaterials.6b00528.

[18] Z. Zhang, S. Xu, Y. Wang, Y. Yu, F. Li, H. Zhu, Y. Shen, S. Huang, S. Guo, Nearinfrared triggered co-delivery of doxorubicin and quercetin by using gold nanocages with tetradecanol to maximize anti-tumor effects on MCF-7/ADR cells, J. Colloid Interface Sci. 509 (2018) 47-57, https://doi.org/10.1016/j.jcis.2017.08. 097.

[19] C. Li, C. Lai, Q. Qiu, X. Luo, L. Hu, H. Zheng, Y. Lu, M. Liu, H. Zhang, X. Liu, Y. Deng, Y. Song, Dual-Ligand modification of PEGylated Liposomes Used for Targeted Doxorubicin Delivery to enhance Anticancer Efficacy, AAPS PharmSciTech 20 (2019) 1-13, https://doi.org/10.1208/s12249-019-1385-0.

[20] J. Ding, F. Shi, C. Xiao, L. Lin, L. Chen, C. He, X. Zhuang, X. Chen, One-step preparation of reduction-responsive poly(ethylene glycol)-poly(amino acid)s nanogels as efficient intracellular drug delivery platforms, Polym. Chem. 2 (2011) 2857-2864, https://doi.org/10.1039/c1py00360g.

[21] X. Li, M. Takashima, E. Yuba, A. Harada, K. Kono, PEGylated PAMAM dendrimerdoxorubicin conjugate-hybridized gold nanorod for combined photothermal-chemotherapy, Biomaterials 35 (2014) 6576-6584, https://doi.org/10.1016/j. biomaterials.2014.04.043.

[22] Y. Zhan, M. Gonçalves, P. Yi, D. Capelo, Y. Zhang, J. Rodrigues, C. Liu, H. Tomás, Y. Li, P. He, Thermo/redox/pH-triple sensitive poly (N-isopropylacrylamide-coacrylic acid) nanogels for anticancer drug delivery, J. Mater. Chem. B 3 (2015) 4221-4230, https://doi.org/10.1039/C5TB00468C.

[23] F. Benyettou, R. Rezgui, F. Ravaux, T. Jaber, K. Blumer, M. Jouiad, L. Motte, J.C. Olsen, C. Platas-Iglesias, M. Magzoub, A. Trabolsi, Synthesis of silver nanoparticles for the dual delivery of doxorubicin and alendronate to cancer cells, J. Mater. Chem. B 3 (2015) 7237-7245, https://doi.org/10.1039/C5TB00994D.

[24] J. Nie, Y. Wang, W. Wang, In vitro and in vivo evaluation of stimuli-responsive vesicle from PEGylated hyperbranched PAMAM-doxorubicin conjugate for gastric cancer therapy, Int. J. Pharm. 509 (2016) 168-177, https://doi.org/10.1016/j. ijpharm.2016.05.021.

[25] Y. Ma, X. Fan, L. Li, pH-sensitive polymeric micelles formed by doxorubicin conjugated prodrugs for co-delivery of doxorubicin and paclitaxel, Carbohydr. Polym. 137 (2016) 19-29, https://doi.org/10.1016/j.carbpol.2015.10.050.

[26] L. Shang, Q. Wang, K. Chen, J. Qu, J. Lin, J. Luo, Q. Zhou, Preparation of polydopamine based redox-sensitive magnetic nanoparticles for doxorubicin delivery and MRI detection, J. Bioresour. Bioprod. 2 (2017) 67-72, https://doi.org/10. $21967 /$ jbb.v2i2.135.

[27] S.P. Kuruvilla, G. Tiruchinapally, A.C. Crouch, M.E.H. ElSayed, J.M. Greve, Dendrimer-doxorubicin conjugates exhibit improved anticancer activity and reduce doxorubicin-induced cardiotoxicity in a murine hepatocellular carcinoma model, PLoS ONE 12 (2017) e0181944, , https://doi.org/10.1371/journal.pone. 0181944.

[28] W.J. Yang, P. Zhou, L. Liang, Y. Cao, J. Qiao, X. Li, Z. Teng, L. Wang, Nanogelincorporated injectable hydrogel for synergistic therapy based on sequential local delivery of combretastatin-A4 phosphate (CA4P) and doxorubicin (DOX), ACS Appl. Mater. Interfaces 10 (2018) 18560-18573, https://doi.org/10.1021/acsami. 8 b04394.

[29] V. Kozlovskaya, F. Liu, Y. Yang, K. Ingle, S. Qian, G.V. Halade, V.S. Urban, E. Kharlampieva, Temperature-responsive polymersomes of poly(3-methyl-N-vinylcaprolactam)- block-poly(N-vinylpyrrolidone) to decrease doxorubicin-induced cardiotoxicity, Biomacromolecules 20 (2019) 3989-4000, https://doi.org/10. 1021/acs.biomac.9b01026.

[30] D.-H. Kim, D.C. Martin, Sustained release of dexamethasone from hydrophilic matrices using PLGA nanoparticles for neural drug delivery, Biomaterials 27 (2006) 3031-3037, https://doi.org/10.1016/j.biomaterials.2005.12.021.

[31] J. Xuan, Y. Yan, D.H. Oh, Y.K. Choi, C.S. Yong, H.-G. Choi, Development of thermo-sensitive injectable hydrogel with sustained release of doxorubicin: rheological characterization and in vivo evaluation in rats, Drug Deliv. 18 (2011) 305-311, https://doi.org/10.3109/10717544.2010.544690.

[32] M. Gonçalves, P. Figueira, D. Maciel, J. Rodrigues, X. Qu, C. Liu, H. Tomás, Y. Li, pH-sensitive Laponite ${ }^{\circledast} /$ doxorubicin/alginate nanohybrids with improved antic ancer efficacy, Acta Biomater. 10 (2014) 300-307, https://doi.org/10.1016/j. actbio.2013.09.013.

[33] M. Gonçalves, P. Figueira, D. Maciel, J. Rodrigues, X. Shi, H. Tomás, Y. Li, Antitumor efficacy of doxorubicin-loaded laponite/alginate hybrid hydrogels, Macromol. Biosci. 14 (2014) 110-120, https://doi.org/10.1002/mabi.201300241.

[34] X.L. Guo, X.X. Kang, Y.Q. Wang, X.J. Zhang, C.J. Li, Y. Liu, L.B. Du, Co-delivery of cisplatin and doxorubicin by covalently conjugating with polyamidoamine dendrimer for enhanced synergistic cancer therapy, Acta Biomater. 84 (2019) 367-377, https://doi.org/10.1016/j.actbio.2018.12.007.

[35] S. Vrignaud, N. Anton, P. Gayet, J.-P. Benoit, P. Saulnier, Reverse micelle-loaded lipid nanocarriers: a novel drug delivery system for the sustained release of doxorubicin hydrochloride, Eur. J. Pharm. Biopharm. 79 (2011) 197-204, https://doi. org/10.1016/j.ejpb.2011.02.015.

[36] L. Qin, F. Zhang, X. Lu, X. Wei, J. Wang, X. Fang, D. Si, Y. Wang, C. Zhang, 
R. Yang, C. Liu, W. Liang, Polymeric micelles for enhanced lymphatic drug delivery to treat metastatic tumors, J. Control. Release 171 (2013) 133-142, https:// doi.org/10.1016/j.jconrel.2013.07.005.

[37] J. Zhu, X. Xu, M. Hu, L. Qiu, Co-encapsulation of combretastatin-A4 phosphate and doxorubicin in polymersomes for synergistic therapy of nasopharyngeal epidermal carcinoma, J. Biomed. Nanotechnol. 11 (2015) 997-1006, https://doi.org/ 10.1166/jbn.2015.2010.

[38] J. Sun, Y. Song, M. Lu, X. Lin, Y. Liu, S. Zhou, Y. Su, Y. Deng, Evaluation of the antitumor effect of dexamethasone palmitate and doxorubicin co-loaded liposomes modified with a sialic acid-octadecylamine conjugate, Eur. J. Pharm. Sci 93 (2016) 177-183, https://doi.org/10.1016/j.ejps.2016.08.029.

[39] K.M. Camacho, S. Menegatti, D.R. Vogus, A. Pusuluri, Z. Fuchs, M. Jarvis, M. Zakrewsky, M.A. Evans, R. Chen, S. Mitragotri, DAFODIL: a novel liposomeencapsulated synergistic combination of doxorubicin and $5 \mathrm{FU}$ for low dose chemotherapy, J. Control. Release 229 (2016) 154-162, https://doi.org/10.1016/j. jconrel.2016.03.027.

[40] D. Zhu, S. Wu, C. Hu, Z. Chen, H. Wang, F. Fan, Y. Qin, C. Wang, H. Sun, X. Leng, D. Kong, L. Zhang, Folate-targeted polymersomes loaded with both paclitaxel and doxorubicin for the combination chemotherapy of hepatocellular carcinoma, Acta Biomater. 58 (2017) 399-412, https://doi.org/10.1016/j.actbio.2017.06.017.

[41] H. Jung, H.-M. Kim, Y. Bin Choy, S.-J. Hwang, J.-H. Choy, Itraconazole-Laponite: kinetics and mechanism of drug release, Appl. Clay Sci. 40 (2008) 99-107, https:// doi.org/10.1016/j.clay.2007.09.002.

[42] X. Li, Z. Yang, K. Yang, Y. Zhou, X. Chen, Y. Zhang, F. Wang, Y. Liu, L. Ren, SelfAssembled polymeric micellar nanoparticles as nanocarriers for poorly soluble anticancer drug ethaselen, Nanoscale Res. Lett. 4 (2009) 1502-1511, https://doi. org/10.1007/s11671-009-9427-2.

[43] A.S. Ertürk, M.U. Gürbüz, M. Tülü, The effect of PAMAM dendrimer concentration, generation size and surface functional group on the aqueous solubility of candesartan cilexetil, Pharm. Dev. Technol. (2016), https://doi.org/10.1080/10837450. 2016.1219372

[44] A. Agarwal, U. Gupta, A. Asthana, N.K. Jain, Dextran conjugated dendritic nanoconstructs as potential vectors for anti-cancer agent, Biomaterials 30 (2009) 3588-3596, https://doi.org/10.1016/j.biomaterials.2009.03.016.

[45] T.-H. Kim, C.W. Mount, W.R. Gombotz, S.H. Pun, The delivery of doxorubicin to 3D multicellular spheroids and tumors in a murine xenograft model using tumorpenetrating triblock polymeric micelles, Biomaterials 31 (2010) 7386-7397, https://doi.org/10.1016/j.biomaterials.2010.06.004.

[46] D. Maciel, P. Figueira, S. Xiao, D. Hu, X. Shi, J. Rodrigues, H. Tomás, Y. Li, Redoxresponsive alginate nanogels with enhanced anticancer cytotoxicity, Biomacromolecules 14 (2013) 3140-3146, https://doi.org/10.1021/bm400768m.

[47] T. Wei, J. Liu, H. Ma, Q. Cheng, Y. Huang, J. Zhao, S. Huo, X. Xue, Z. Liang, X.J. Liang, Functionalized nanoscale micelles improve drug delivery for cancer therapy in vitro and in vivo, Nano Lett. 13 (2013) 2528-2534, https://doi.org/10. 1021/nl400586t

[48] J. Xu, Q. Zhao, Y. Jin, L. Qiu, High loading of hydrophilic/hydrophobic doxorubicin into polyphosphazene polymersome for breast cancer therapy, Nanomedicine 10 (2014) 349-358, https://doi.org/10.1016/j.nano.2013.08.004.

[49] Y. Zhao, W. Ren, T. Zhong, S. Zhang, D. Huang, Y. Guo, X. Yao, C. Wang, W. Q. Zhang, X. Zhang, Q. Zhang, Tumor-specific $\mathrm{pH}$-responsive peptide-modified $\mathrm{pH}$ sensitive liposomes containing doxorubicin for enhancing glioma targeting and anti-tumor activity, J. Control. Release 222 (2016) 56-66, https://doi.org/10. 1016/j.jconrel.2015.12.006

[50] C. Emilienne Soma, C. Dubernet, D. Bentolila, S. Benita, P. Couvreur, Reversion of multidrug resistance by co-encapsulation of doxorubicin and cyclosporin $\mathrm{A}$ in polyalkylcyanoacrylate nanoparticles, Biomaterials 21 (2000) 1-7, https://doi. org/10.1016/S0142-9612(99)00125-8.

[51] J. Shen, Q. He, Y. Gao, J. Shi, Y. Li, Mesoporous silica nanoparticles loading doxorubicin reverse multidrug resistance: performance and mechanism, Nanoscale 3 (2011) 4314-4322, https://doi.org/10.1039/c1nr10580a.

[52] Y. Lu, Y. Ma, C. Feng, D. Zhu, J. Liu, L. Chen, S. Liang, C. Dong, Co-delivery of cyclopamine and doxorubicin mediated by bovine serum albumin nanoparticles reverses doxorubicin resistance in breast cancer by down-regulating p-glycoprotein expression, J. Cancer 10 (2019) 2357-2368, https://doi.org/10.7150/jca. 30323.

[53] Y. Xiao, H. Hong, V.Z. Matson, A. Javadi, W. Xu, Y. Yang, Y. Zhang, J.W. Engle, R.J. Nickles, W. Cai, D.A. Steeber, S. Gong, Gold nanorods conjugated with dox orubicin and cRGD for combined anti-cancer drug delivery and PET imaging, Theranostics 2 (2012) 757-768, https://doi.org/10.7150/thno.4756.

[54] M. Howell, J. Mallela, C. Wang, S. Ravi, S. Dixit, U. Garapati, S. Mohapatra, Manganese-loaded lipid-micellar theranostics for simultaneous drug and gene delivery to lungs, J. Control. Release 167 (2013) 210-218, https://doi.org/10. 1016/j.jconrel.2013.01.029.

[55] Y.-H. Shih, C.-L. Peng, P.-F. Chiang, W.-J. Lin, T.-Y. Luo, M.-J. Shieh, Therapeutic and scintigraphic applications of polymeric micelles: Combination of chemotherapy and radiotherapy in hepatocellular carcinoma, Int. J. Nanomedicine 10 (2015) 7443-7454, https://doi.org/10.2147/IJN.S91008.

[56] S. Rizzitelli, P. Giustetto, D. Faletto, D. Delli Castelli, S. Aime, E. Terreno, The release of Doxorubicin from liposomes monitored by MRI and triggered by a combination of US stimuli led to a complete tumor regression in a breast cancer mouse model, J. Control. Release 230 (2016) 57-63, https://doi.org/10.1016/j. jconrel.2016.03.040.

[57] J. Zhu, G. Wang, C.S. Alves, H. Tomás, Z. Xiong, M. Shen, J. Rodrigues, X. Shi, Multifunctional dendrimer-entrapped gold nanoparticles Conjugated with Doxorubicin for pH-Responsive Drug Delivery and Targeted Computed tomography imaging, Langmuir 34 (2018) 12428-12435, https://doi.org/10.1021/acs. langmuir.8b02901

[58] C. Carvalho, R.X. Santos, S. Cardoso, S. Correia, P.J. Oliveira, M.S. Santos, P.I. Moreira, Doxorubicin: the good, the bad and the ugly effect, Curr. Med. Chem. 16 (2009) 3267-3285, https://doi.org/10.2174/092986709788803312.

[59] C.F. Thorn, C. Oshiro, S. Marsh, T. Hernandez-Boussard, H. McLeod, T.E. Klein, R.B. Altman, Doxorubicin pathways: pharmacodynamics and adverse effects, Pharmacogenet. Genom. 21 (2011) 440-446, https://doi.org/10.1097/FPC 0b013e32833ffb56.Doxorubicin.

[60] G.N. Hortobágyi, Anthracyclines in the treatment of cancer an overview, Drugs 54 (1997) 1-7, https://doi.org/10.2165/00003495-199700544-00003.

[61] F. Yang, S.S. Teves, C.J. Kemp, S. Henikoff, Doxorubicin, DNA torsion, and chromatin dynamics, Biochim. Biophys. Acta 1845 (2014) 84-89, https://doi.org/ 10.1016/j.bbcan.2013.12.002.

[62] O. Tacar, P. Sriamornsak, C.R. Dass, Doxorubicin: an update on anticancer molecular action, toxicity and novel drug delivery systems, J. Pharm. Pharmacol. 65 (2013) 157-170, https://doi.org/10.1111/j.2042-7158.2012.01567.x.

[63] F. Arcamone, G. Cassinelli, G. Franceschi, S. Penco, C. Pol, S. Redaelli, A. Selva, Structure and physicochemical Properties of Adriamycin (Doxorubicin), Int. Symp. Adriamycin. (1972) 9-22, https://doi.org/10.1007/978-3-642-95227-2_2.

[64] F. Arcamone, Discovery and development of Doxorubicin, Med. Chem. (Los. Angeles), Academic Press, New York, 1981, pp. 1-47, , https://doi.org/10.1016/ B978-0-12-059280-7.50007-9.

[65] F. Arcamone, Antitumor anthracyclines: recent developments, Med. Res. Rev. 4 (1984) 153-188, https://doi.org/10.1002/med.2610040203.

[66] M. Cagel, E. Grotz, E. Bernabeu, M.A. Moretton, D.A. Chiappetta, Doxorubicin: nanotechnological overviews from bench to bedside, Drug Discov. Today 22 (2017) 270-281, https://doi.org/10.1016/j.drudis.2016.11.005.

[67] I. Borišev, J. Mrdanovic, D. Petrovic, M. Seke, D. Jović, B. Srdenović, N. Latinovic, A. Djordjevic, Nanoformulations of doxorubicin: how far have we come and where do we go from here? Nanotechnology 29 (2018), https://doi.org/10.1088/1361$6528 /$ aac7dd.

[68] Y. Barenholz, Doxil ${ }^{\oplus}$ - The first FDA-approved nano-drug: lessons learned, J. Control. Release 160 (2012) 117-134, https://doi.org/10.1016/j.jconrel.2012.03. 020.

[69] L. Miao, C.M. Lin, L. Huang, Stromal barriers and strategies for the delivery of nanomedicine to desmoplastic tumors, J. Control. Release 219 (2015) 192-204, https://doi.org/10.1016/j.jconrel.2015.08.017.

[70] C. von Roemeling, W. Jiang, C.K. Chan, I.L. Weissman, B.Y.S. Kim, Breaking down the barriers to precision cancer nanomedicine, Trends Biotechnol. 35 (2017) 159-171, https://doi.org/10.1016/j.tibtech.2016.07.006.

[71] R. van Furth, Z.A. Cohn, J.G. Hirsch, J.H. Humphrey, W.G. Spector, H.L. Langevoort, The mononuclear phagocyte system: a new classification of macrophages, monocytes, and their precursor cells, Bull. World Health Organ. 46 (1972) 845-852.

[72] S. Zhu, M. Hong, G. Tang, L. Qian, J. Lin, Y. Jiang, Y. Pei, Partly PEGylated polyamidoamine dendrimer for tumor-selective targeting of doxorubicin: the effects of PEGylation degree and drug conjugation style, Biomaterials 31 (2010) 1360-1371, https://doi.org/10.1016/j.biomaterials.2009.10.044.

[73] E. Allard-Vannier, S. Cohen-Jonathan, J. Gautier, K. Hervé-Aubert, E. Munnier, M. Soucé, P. Legras, C. Passirani, I. Chourpa, Pegylated magnetic nanocarriers for doxorubicin delivery: a quantitative determination of stealthiness in vitro and in vivo, Eur. J. Pharm. Biopharm. 81 (2012) 498-505, https://doi.org/10.1016/j. ejpb.2012.04.002.

[74] K.T. Al-Jamal, W.T. Al-Jamal, J.T.-W. Wang, N. Rubio, J. Buddle, D. Gathercole, M. Zloh, K. Kostarelos, Cationic poly-L-Lysine dendrimer complexes doxorubicin and delays tumor growth in vitro and in vivo, ACS Nano 7 (2013) 1905-1917, https://doi.org/10.1021/nn305860k.

[75] N.G. Yabbarov, E.D. Nikolskaya, O.A. Zhunina, I.G. Kondrasheva, I.A. Zamulaeva, E.S. Severin, Polyamidoamine dendrimers with different surface charge as carriers in anticancer drug delivery, Russ. J. Bioorganic Chem. 43 (2017) 155-162, https://doi.org/10.1134/S1068162017020182.

[76] O. Gamucci, A. Bertero, M. Gagliardi, G. Bardi, Biomedical nanoparticles: overview of their surface immune-compatibility, Coatings 4 (2014) 139-159, https:// doi.org/10.3390/coatings4010139.

[77] S.K. Hobbs, W.L. Monsky, F. Yuan, W.G. Roberts, L. Griffith, V. ladimir P. Torchilin, R.K. Jain, Regulation of transport pathways in tumor vessels: role of tumor type and microenvironment, Proc. Natl. Acad. Sci. U. S. A. 95 (1998) 4607-4612, https://doi.org/10.1073/pnas.95.8.4607.

[78] F. Danhier, To exploit the tumor microenvironment: since the EPR effect fails in the clinic, what is the future of nanomedicine? J. Control. Release 244 (2016) 108-121, https://doi.org/10.1016/j.jconrel.2016.11.015.

[79] R.K. Jain, Transport of molecules across tumor vasculature, Cancer Metastasis Rev. 6 (1987) 559-593, https://doi.org/10.1007/BF00047468.

[80] E.M. Renkin, Cellular and intercellular transport pathways in exchange vessels, Am. Rev. Respir. Dis. 146 (1992) S28-S31, https://doi.org/10.1164/ajrccm/146. 5 Pt 2.S28.

[81] H. He, Y. Li, X.-R. Jia, J. Du, X. Ying, W.-L. Lu, J.-N. Lou, Y. Wei, PEGylated Poly (amidoamine) dendrimer-based dual-targeting carrier for treating brain tumors, Biomaterials 32 (2011) 478-487, https://doi.org/10.1016/j.biomaterials.2010. 09.002.

[82] Z. Pang, H. Gao, Y. Yu, L. Guo, J. Chen, S. Pan, J. Ren, Z. Wen, X. Jiang, Enhanced intracellular delivery and chemotherapy for glioma rats by transferrin-conjugated biodegradable polymersomes loaded with doxorubicin, Bioconjug. Chem. 22 (2011) 1171-1180, https://doi.org/10.1021/bc200062q.

[83] J. Ren, S. Shen, D. Wang, Z. Xi, L. Guo, Z. Pang, Y. Qian, X. Sun, X. Jiang, The targeted delivery of anticancer drugs to brain glioma by PEGylated oxidized multi- 
walled carbon nanotubes modified with angiopep-2, Biomaterials 33 (2012) 3324-3333, https://doi.org/10.1016/j.biomaterials.2012.01.025.

[84] Y.C. Kuo, I.H. Lee, Delivery of doxorubicin to glioblastoma multiforme in vitro using solid lipid nanoparticles with surface aprotinin and melanotransferrin antibody for enhanced chemotherapy, J. Taiwan Inst. Chem. Eng. 61 (2016) 32-45, https://doi.org/10.1016/j.jtice.2015.12.012.

[85] S. Li, D. Amat, Z. Peng, S. Vanni, S. Raskin, G. De Angulo, A.M. Othman, R.M. Graham, R.M. Leblanc, Transferrin conjugated nontoxic carbon dots for doxorubicin delivery to target pediatric brain tumor cells, Nanoscale 8 (2016) 16662-16669, https://doi.org/10.1039/C6NR05055G.

[86] P.J. Gaillard, C.C. Visser, C.C.M. Appeldoorn, J. Rip, Enhanced brain drug delivery: safely crossing the blood-brain barrier, Drug Discov. Today Technol. 9 (2012) e155-e160, https://doi.org/10.1016/j.ddtec.2011.12.002.

[87] W.M. Pardridge, CSF, blood-brain barrier, and brain drug delivery, Expert Opin. Drug Deliv. 13 (2016) 963-975, https://doi.org/10.1517/17425247.2016. 1171315.

[88] S. Nallanthighal, J.P. Heiserman, D.-J. Cheon, The role of the extracellular matrix in cancer stemness, Front. Cell Dev. Biol. 7 (2019) 1-14, https://doi.org/10.3389/ fcell.2019.00086.

[89] R.K. Jain, T. Stylianopoulos, Delivering nanomedicine to solid tumors, Nat. Rev. Clin. Oncol. 7 (2010) 653-664, https://doi.org/10.1038/nrclinonc.2010.139.

[90] L.M. Bareford, P.W. Swaan, Endocytic mechanisms for targeted drug delivery, Adv. Drug Deliv. Rev. 59 (2007) 748-758, https://doi.org/10.1016/j.addr.2007. 06.008.

[91] B.P. Mahoney, N. Raghunand, B. Baggett, R.J. Gillies, Tumor acidity, ion trapping and chemotherapeutics I. Acid $\mathrm{pH}$ affects the distribution of chemotherapeutic agents in vitro, Biochem. Pharmacol. 66 (2003) 1207-1218, https://doi.org/10. 1016/S0006-2952(03)00467-2.

[92] G. Wang, D. Maciel, Y. Wu, J. Rodrigues, X. Shi, Y. Yuan, C. Liu, H. Tomás, Y. Li, Amphiphilic polymer-mediated formation of laponite-based nanohybrids with robust stability and $\mathrm{pH}$ sensitivity for anticancer drug delivery, ACS Appl. Mater. Interfaces 6 (2014) 16687-16695, https://doi.org/10.1021/am5032874.

[93] M. Gonçalves, P. Figueira, D. Maciel, J. Rodrigues, X. Shi, H. Tomás, Y. Li, Antitumor efficacy of doxorubicin-loaded laponite/alginate hybrid hydrogels, Macromol. Biosci. 14 (2014) 110-120, https://doi.org/10.1002/mabi.201300241.

[94] S. Zhu, M. Hong, L. Zhang, G. Tang, Y. Jiang, Y. Pei, PEGylated PAMAM dendrimer-doxorubicin conjugates: in vitro evaluation and in vivo tumor accumulation, Pharm. Res. 27 (2010) 161-174, https://doi.org/10.1007/s11095-0099992-1.

[95] G. Chen, D. Li, J. Li, X. Cao, J. Wang, X. Shi, R. Guo, Targeted doxorubicin delivery to hepatocarcinoma cells by lactobionic acid-modified laponite nanodisks, New J. Chem. 39 (2015) 2847-2855, https://doi.org/10.1039/C4NJ01916D.

[96] W. Hu, L. Qiu, L. Cheng, Q. Hu, Y. Liu, Z. Hu, D. Chen, L. Cheng, Redox and pH dual responsive poly(amidoamine) dendrimer-poly(ethylene glycol) conjugates for intracellular delivery of doxorubicin, Acta Biomater. 36 (2016) 241-253, https:// doi.org/10.1016/j.actbio.2016.03.027.

[97] T. Jiang, G. Chen, X. Shi, R. Guo, Hyaluronic acid-decorated laponite ${ }^{\circledast}$ nanocomposites for targeted anticancer drug delivery, Polymers (Basel). 11 (2019) 137, https://doi.org/10.3390/polym11010137.

[98] T. Lammers, F. Kiessling, W.E. Hennink, G. Storm, Drug targeting to tumors: principles, pitfalls and (pre-) clinical progress, J. Control. Release 161 (2012) 175-187, https://doi.org/10.1016/j.jconrel.2011.09.063.

[99] Clinical and pharmacological study with 2B3-101 in patients with breast cancer and leptomeningeal metastases, Clinicaltrials.Gov. (2013), https://www. clinicaltrials.gov/ct2/show/NCT01818713?term $=2$ B3-101\&rank $=1$ (accessed June 17, 2019).

[100] B.M. Kerklaan, A. Jager, P. Aftimos, V. Dieras, S. Altintas, C. Anders, M. Arnedos, H. Gelderblom, P. Soetekouw, W. Gladdines, P. Gaillard, C. de Sousa, A. Awada, J. Schellens, M. van Linde, D. Brandsma, NT-23. Phase 1/2A study of glutathione pegylated liposomal doxorubicin (2B3-101) in breast cancer patients with brain metastases (BCBM) or recurretn high grade gliomas (HGG), Neuro Oncol. 16 (2014), https://doi.org/10.1093/neuonc/nou265.21 v163-v163.

[101] C. Mamot, R. Ritschard, A. Wicki, G. Stehle, T. Dieterle, L. Bubendorf, C. Hilker, S. Deuster, R. Herrmann, C. Rochlitz, Tolerability, safety, pharmacokinetics, and efficacy of doxorubicin-loaded anti-EGFR immunoliposomes in advanced solid tumours: a phase 1 dose-escalation study, Lancet Oncol. 13 (2012) 1234-1241, https://doi.org/10.1016/S1470-2045(12)70476-X.

[102] K. Miller, J. Cortes, S.A. Hurvitz, I.E. Krop, D. Tripathy, S. Verma, K. Riahi, J.G. Reynolds, T.J. Wickham, I. Molnar, D.A. Yardley, HERMIONE: a randomized Phase 2 trial of MM-302 plus trastuzumab versus chemotherapy of physician's choice plus trastuzumab in patients with previously treated, anthracycline-naïve, HER2-positive, locally advanced/metastatic breast cancer, BioMed Cent. Cancer. 16 (2016) 352, , https://doi.org/10.1186/s12885-016-2385-z.

[103] L.W. Seymour, D.R. Ferry, D. Anderson, S. Hesslewood, P.J. Julyan, R. Poyner, J. Doran, A.M. Young, S. Burtles, D.J. Kerr, Hepatic drug targeting: phase I evaluation of polymer-bound doxorubicin, J. Clin. Oncol. 20 (2002) 1668-1676, https://doi.org/10.1200/JCO.2002.20.6.1668.

[104] Y. Zhan, M. Gonçalves, P. Yi, D. Capelo, Y. Zhang, J. Rodrigues, C. Liu, H. Tomás, Y. Li, P. He, Thermo/Redox/pH-stimulative nanogel delivery systems, Nanomedicine 12 (2016) 567, https://doi.org/10.1016/j.nano.2015.12.337.

[105] S. Xiao, R. Castro, D. Maciel, M. Gonçalves, X. Shi, J. Rodrigues, H. Tomás, Fine tuning of the $\mathrm{pH}$-sensitivity of laponite-doxorubicin nanohybrids by polyelectrolyte multilayer coating, Mater. Sci. Eng. C 60 (2016) 348-356, https://doi.org/ 10.1016/j.msec.2015.11.051.

[106] L.M. Kaminskas, B.D. Kelly, V.M. McLeod, G. Sberna, D.J. Owen, B.J. Boyd, C.J.H. Porter, Characterisation and tumour targeting of PEGylated polylysine dendrimers bearing doxorubicin via a $\mathrm{pH}$ labile linker, J. Control. Release 152 (2011) 241-248, https://doi.org/10.1016/j.jconrel.2011.02.005.

[107] Y. Pu, S. Chang, H. Yuan, G. Wang, B. He, Z. Gu, The anti-tumor efficiency of poly (L-glutamic acid) dendrimers with polyhedral oligomeric silsesquioxane cores, Biomaterials 34 (2013) 3658-3666, https://doi.org/10.1016/j.biomaterials.2013. 01.082 .

[108] E. Ruiz-Hernández, M. Hess, G.J. Melen, B. Theek, M. Talelli, Y. Shi, B. Ozbakir, E.A. Teunissen, M. Ramírez, D. Moeckel, F. Kiessling, G. Storm, H.W. Scheeren, W.E. Hennink, A.A. Szalay, J. Stritzker, T. Lammers, PEG-pHPMAm-based polymeric micelles loaded with doxorubicin-prodrugs in combination antitumor therapy with oncolytic vaccinia viruses, Polym. Chem. 5 (2014) 1674-1681, https://doi.org/10.1039/C3PY01097J.

[109] K.Y.J. Lee, Y. Wang, S. Nie, In vitro study of a pH-sensitive multifunctional doxorubicin-gold nanoparticle system: therapeutic effect and surface enhanced Raman scattering, R. Soc. Chem. Adv. 5 (2015) 65651-65659, https://doi.org/10. 1039/C5RA09872F.

[110] M. Marcinkowska, E. Sobierajska, M. Stanczyk, A. Janaszewska, A. Chworos, B. Klajnert-Maculewicz, Conjugate of PAMAM dendrimer, doxorubicin and monoclonal antibody-trastuzumab: the new approach of a well-known strategy, Polymers (Basel). 10 (2018) 1-11, https://doi.org/10.3390/polym10020187.

[111] K.E. Uhrich, S.M. Cannizzaro, R.S. Langer, K.M. Shakesheff, Polymeric systems for controlled drug release, Chem. Rev. 99 (1999) 3181-3198, https://doi.org/10. 1021/cr940351u.

[112] F. Danhier, E. Ansorena, J.M. Silva, R. Coco, A. Le Breton, V. Préat, PLGA-based nanoparticles: an overview of biomedical applications, J. Control. Release 161 (2012) 505-522, https://doi.org/10.1016/j.jconrel.2012.01.043.

[113] L.-P. Wu, M. Ficker, J.B. Christensen, P.N. Trohopoulos, S.M. Moghimi, Dendrimers in medicine: therapeutic concepts and pharmaceutical challenges, Bioconjug. Chem. 26 (2015) 1198-1211, https://doi.org/10.1021/acs. bioconjchem.5b00031.

[114] R. Esfand, D.A. Tomalia, Poly(amidoamine) (PAMAM) dendrimers: from biomimicry to drug delivery and biomedical applications, Drug Discov. Today 6 (2001) 427-436, https://doi.org/10.1016/S1359-6446(01)01757-3.

[115] K.E. Sapsford, W.R. Algar, L. Berti, K.B. Gemmill, B.J. Casey, E. Oh, M.H. Stewart, I.L. Medintz, Functionalizing nanoparticles with biological molecules: developing chemistries that facilitate nanotechnology, Chem. Rev. 113 (2013) 1904-2074, https://doi.org/10.1021/cr300143v.

[116] A. Almutairi, R. Rossin, M. Shokeen, A. Hagooly, A. Ananth, B. Capoccia, S. Guillaudeu, D. Abendschein, C.J. Anderson, M.J. Welch, J.M.J. Fréchet, Biodegradable dendritic positron-emitting nanoprobes for the noninvasive imaging of angiogenesis, Proc. Natl. Acad. Sci. U. S. A. 106 (2009) 685-690, https:// doi.org/10.1073/pnas.0811757106.

[117] A. Ghai, B. Singh, P. Panwar Hazari, M.K. Schultz, A. Parmar, P. Kumar, S. Sharma, D. Dhawan, A. Kumar Mishra, Radiolabeling optimization and characterization of ${ }^{68} \mathrm{Ga}$ labeled DOTA-polyamido-amine dendrimer conjugate - animal biodistribution and PET imaging results, Appl. Radiat. Isot. 105 (2015) 40-46, https://doi. org/10.1016/j.apradiso.2015.07.021.

[118] C. Wängler, S. Maschauer, O. Prante, M. Schäfer, R. Schirrmacher, P. Bartenstein, M. Eisenhut, B. Wängler, Multimerization of cRGD peptides by click chemistry: synthetic strategies, chemical limitations, and influence on biological properties, Chem. Bio Chem. 11 (2010) 2168-2181, https://doi.org/10.1002/cbic. 201000386.

[119] K. Tanaka, E.R.O. Siwu, K. Minami, K. Hasegawa, S. Nozaki, Y. Kanayama, K. Koyama, W.C. Chen, J.C. Paulson, Y. Watanabe, K. Fukase, Noninvasive imaging of dendrimer-type N-glycan clusters: in vivo dynamics dependence on oligosaccharide structure, Angew. Chem. Int. Ed. 49 (2010) 8195-8200, https://doi. org/10.1002/anie.201000892.

[120] L. Trembleau, M. Simpson, R.W. Cheyne, I. Escofet, M.V.C.A.L. Appleyard, K. Murray, S. Sharp, A.M. Thompson, T.A.D. Smith, Development of $18 \mathrm{~F}$-fluorinatable dendrons and their application to cancer cell targeting, New J. Chem. 35 (2011) 2496-2502, https://doi.org/10.1039/c1nj20417c.

[121] J.M. Criscione, L.W. Dobrucki, Z.W. Zhuang, X. Papademetris, M. Simons, A.J. Sinusas, T.M. Fahmy, Development and application of a multimodal contrast agent for SPECT/CT hybrid imaging, Bioconjug. Chem. 22 (2011) 1784-1792, https://doi.org/10.1021/bc200162r.

[122] K. Li, S. Wen, A.C. Larson, M. Shen, Z. Zhang, Q. Chen, X. Shi, G. Zhang, Multifunctional dendrimer-based nanoparticles for in vivo MR/CT dual-modal molecular imaging of breast cancer, Int. J. Nanomedicine 8 (2013) 2589-2600, https://doi.org/10.2147/IJN.S46177.

[123] H. Liu, Y. Xu, S. Wen, Q. Chen, L. Zheng, M. Shen, J. Zhao, G. Zhang, X. Shi, Targeted tumor computed tomography imaging using low-generation dendrimerstabilized gold nanoparticles, Chem. - A Eur. J. 19 (2013) 6409-6416, https://doi. org/10.1002/chem.201204612.

[124] Q. Chen, K. Li, S. Wen, H. Liu, C. Peng, H. Cai, M. Shen, G. Zhang, X. Shi, Targeted $\mathrm{CT} / \mathrm{MR}$ dual mode imaging of tumors using multifunctional dendrimer-entrapped gold nanoparticles, Biomaterials 34 (2013) 5200-5209, https://doi.org/10.1016/ j.biomaterials.2013.03.009.

[125] J.W. Seo, H. Baek, L.M. Mahakian, J. Kusunose, J. Hamzah, E. Ruoslahti, K.W. Ferrara, 64Cu-labeled LyP-1-dendrimer for PET-CT imaging of atherosclerotic plaque, Bioconjug. Chem. 25 (2014) 231-239, https://doi.org/10.1021/ bc400347s.

[126] J.B. Wolinsky, M.W. Grinstaff, Therapeutic and diagnostic applications of dendrimers for cancer treatment, Adv. Drug Deliv. Rev. 60 (2008) 1037-1055, https://doi.org/10.1016/j.addr.2008.02.012.

[127] C.C. Lee, J.A. MacKay, J.M.J. Fréchet, F.C. Szoka, Designing dendrimers for biological applications, Nat. Biotechnol. 23 (2005) 1517-1526, https://doi.org/10. 
$1038 /$ nbt1171

[128] I.J. Majoros, A. Myc, T. Thomas, C.B. Mehta, J.R. Baker, PAMAM dendrimer-based multifunctional conjugate for cancer therapy: Synthesis, characterization, and functionality, Biomacromolecules 7 (2006) 572-579, https://doi.org/10.1021/ bm0506142.

[129] X. Shi, S. Wang, S. Meshinchi, M.E. Van Antwerp, X. Bi, I. Lee, J.R. Baker, Dendrimer-entrapped gold nanoparticles as a platform for cancer-cell targeting and imaging, Small 3 (2007) 1245-1252, https://doi.org/10.1002/smll. 200700054.

[130] X. Shi, S. Wang, H. Sun, J.R. Baker, Improved biocompatibility of surface functionalized dendrimer-entrapped gold nanoparticles, Soft Matter 3 (2007) 71-74, https://doi.org/10.1039/b612972b.

[131] R.B. Kolhatkar, K.M. Kitchens, P.W. Swaan, H. Ghandehari, Surface acetylation of polyamidoamine (PAMAM) dendrimers decreases cytotoxicity while maintaining membrane permeability, Bioconjug. Chem. 18 (2007) 2054-2060, https://doi. org $/ 10.1021 /$ bc0603889.

[132] K. Fant, E.K. Esbjörner, A. Jenkins, M.C. Grossel, P. Lincoln, B. Nordén, Effects of PEGylation and acetylation of PAMAM dendrimers on DNA binding, cytotoxicity and in vitro transfection efficiency, Mol. Pharm. 7 (2010) 1734-1746, https://doi. org/10.1021/mp1001312.

[133] J. Hu, Y. Su, H. Zhang, T. Xu, Y. Cheng, Design of interior-functionalized fully acetylated dendrimers for anticancer drug delivery, Biomaterials 32 (2011) 9950-9959, https://doi.org/10.1016/j.biomaterials.2011.09.016.

[134] W. Hou, S. Wen, R. Guo, S. Wang, X. Shi, Partially acetylated dendrimer-entrapped gold nanoparticles with reduced cytotoxicity for gene delivery applications, J. Nanosci. Nanotechnol. 15 (2015) 4094-4105, https://doi.org/10.1166/jnn.2015. 9618.

[135] Y. Cheng, L. Zhao, Y. Li, T. Xu, Design of biocompatible dendrimers for cancer diagnosis and therapy: current status and future perspectives, Chem. Soc. Rev. 40 (2011) 2673-2703, https://doi.org/10.1039/c0cs00097c.

[136] M.L. Patil, M. Zhang, O. Taratula, O.B. Garbuzenko, H. He, T. Minko, Internally cationic polyamidoamine PAMAM-OH dendrimers for siRNA delivery: effect of the degree of Quaternization and cancer targeting, Biomacromolecules 10 (2009) 258-266, https://doi.org/10.1021/bm8009973.

[137] L. Han, R. Huang, J. Li, S. Liu, S. Huang, C. Jiang, Plasmid pORF-hTRAIL and doxorubicin co-delivery targeting to tumor using peptide-conjugated polyamidoamine dendrimer, Biomaterials 32 (2011) 1242-1252, https://doi.org/10. 1016/j.biomaterials.2010.09.070.

[138] I.-H. Lee, M.K. Yu, I.H. Kim, J.-H. Lee, T.G. Park, S. Jon, A duplex oligodeoxynucleotide-dendrimer bioconjugate as a novel delivery vehicle for doxorubicin in in vivo cancer therapy, J. Control. Release 155 (2011) 88-95, https://doi.org/10. 1016/j.jconrel.2010.09.008.

[139] X. He, C.S. Alves, N. Oliveira, J. Rodrigues, J. Zhu, I. Bányai, H. Tomás, X. Shi, RGD peptide-modified multifunctional dendrimer platform for drug encapsulation and targeted inhibition of cancer cells, Colloids Surf. B: Biointerfaces 125 (2015) 82-89, https://doi.org/10.1016/j.colsurfb.2014.11.004.

[140] X. Xu, J. Li, S. Han, C. Tao, L. Fang, Y. Sun, J. Zhu, Z. Liang, F. Li, A novel doxorubicin loaded folic acid conjugated PAMAM modified with borneol, a nature dual-functional product of reducing PAMAM toxicity and boosting BBB penetration, Eur. J. Pharm. Sci. 88 (2016) 178-190, https://doi.org/10.1016/j.ejps.2016. 02.015 .

[141] Z. Zhang, Y. Zhou, Z. Zhou, Y. Piao, N. Kalva, X. Liu, J. Tang, Y. Shen, Synthesis of enzyme-responsive phosphoramidate dendrimers for cancer drug delivery, Polym. Chem. 9 (2018) 438-449, https://doi.org/10.1039/c7py01492a.

[142] F. Wang, X. Cai, Y. Su, J. Hu, Q. Wu, H. Zhang, J. Xiao, Y. Cheng, Reducing cytotoxicity while improving anti-cancer drug loading capacity of polypropylenimine dendrimers by surface acetylation, Acta Biomater. 8 (2012) 4304-4313, https://doi.org/10.1016/j.actbio.2012.07.031.

[143] M. Golshan, M. Salami-Kalajahi, H. Roghani-Mamaqani, M. Mohammadi, Poly (propylene imine) dendrimer-grafted nanocrystalline cellulose: doxorubicin loading and release behavior, Polymer (Guildf). 117 (2017) 287-294, https://doi. org /10.1016/j.polymer.2017.04.047.

[144] J.K. Oh, R. Drumright, D.J. Siegwart, K. Matyjaszewski, The development of microgels/nanogels for drug delivery applications, Prog. Polym. Sci. 33 (2008) 448-477, https://doi.org/10.1016/j.progpolymsci.2008.01.002.

[145] O. Wichterle, D. Lím, Hydrophilic gels for biological use, Nature 185 (1960) 117-118, https://doi.org/10.1038/185117a0.

[146] T.R. Hoare, D.S. Kohane, Hydrogels in drug delivery: progress and challenges, Polymer (Guildf). 49 (2008) 1993-2007, https://doi.org/10.1016/j.polymer. 2008.01.027.

[147] Y. Li, D. Maciel, J. Rodrigues, X. Shi, H. Tomás, Biodegradable polymer nanogels for drug/nucleic acid delivery, Chem. Rev. 115 (2015) 8564-8608, https://doi. org/10.1021/cr500131f.

[148] N.A. Peppas, P. Bures, W. Leobandung, H. Ichikawa, Hydrogels in pharmaceutical formulations, Eur. J. Pharm. Biopharm. 50 (2000) 27-46, https://doi.org/10. 1016/S0939-6411(00)00090-4.

[149] E. Caló, V.V. Khutoryanskiy, Biomedical applications of hydrogels: a review of patents and commercial products, Eur. Polym. J. 65 (2015) 252-267, https://doi. org/10.1016/j.eurpolymj.2014.11.024.

[150] C. Duan, J. Gao, D. Zhang, L. Jia, Y. Liu, D. Zheng, G. Liu, X. Tian, F. Wang, $\mathrm{Q}$. Zhang, Galactose-decorated $\mathrm{pH}$-responsive nanogels for hepatoma-targeted delivery of oridonin, Biomacromolecules 12 (2011) 4335-4343, https://doi.org/ 10.1021/bm201270m.

[151] Y.J. Pan, Y.Y. Chen, D.R. Wang, C. Wei, J. Guo, D.R. Lu, C.C. Chu, C.C. Wang, Redox/pH dual stimuli-responsive biodegradable nanohydrogels with varying responses to dithiothreitol and glutathione for controlled drug release,
Biomaterials 33 (2012) 6570-6579, https://doi.org/10.1016/j.biomaterials.2012. 05.062 .

[152] X. Zhang, K. Zhang, R. Haag, Multi-stage, charge conversional, stimuli-responsive nanogels for therapeutic protein delivery, Biomater. Sci. 3 (2015) 1487-1496, https://doi.org/10.1039/c5bm00171d.

[153] X. Zhang, K. Achazi, R. Haag, Boronate cross-linked ATP- and pH-responsive nanogels for intracellular delivery of anticancer drugs, Adv. Healthc. Mater. 4 (2015) 585-592, https://doi.org/10.1002/adhm.201400550.

[154] H. Yang, Q. Wang, S. Huang, A. Xiao, F. Li, L. Gan, X. Yang, Smart pH/redox dualresponsive Nanogels for on-demand intracellular anticancer drug release, ACS Appl. Mater. Interfaces 8 (2016) 7729-7738, https://doi.org/10.1021/acsami. 6b01602.

[155] S. Chen, Q. Bian, P. Wang, X. Zheng, L. Lv, Z. Dang, G. Wang, Photo, pH and redox multi-responsive nanogels for drug delivery and fluorescence cell imaging, Polym. Chem. 8 (2017) 6150-6157, https://doi.org/10.1039/c7py01424d.

[156] W. Park, K.S. Kim, B. Bae, Y. Kim, K. Na, Cancer cell specific targeting of nanogels from acetylated hyaluronic acid with low molecular weight, Eur. J. Pharm. Sci. 40 (2010) 367-375, https://doi.org/10.1016/j.ejps.2010.04.008.

[157] W. Xiong, W. Wang, Y. Wang, Y. Zhao, H. Chen, H. Xu, X. Yang, Dual temperature/ $\mathrm{pH}$-sensitive drug delivery of poly(N-isopropylacrylamide-co-acrylic acid) nanogels conjugated with doxorubicin for potential application in tumor hyperthermia therapy, Colloids Surf. B: Biointerfaces 84 (2011) 447-453, https://doi.org/10. 1016/j.colsurfb.2011.01.040.

[158] D. Maciel, P. Figueira, S. Xiao, D. Hu, X. Shi, J. Rodrigues, H. Tomás, Y. Li, Redoxresponsive alginate nanogels with enhanced anticancer cytotoxicity, Biomacromolecules 14 (2013) 3140-3146, https://doi.org/10.1021/bm400768m.

[159] M. Gonçalves, D. Maciel, D. Capelo, S. Xiao, W. Sun, X. Shi, J. Rodrigues, H. Tomás, Y. Li, Dendrimer-assisted formation of fluorescent nanogels for drug delivery and intracellular imaging, Biomacromolecules 15 (2014) 492-499, https://doi.org/10.1021/bm401400r.

[160] S. Manchun, C.R. Dass, K. Cheewatanakornkool, P. Sriamornsak, Enhanced antitumor effect of $\mathrm{pH}$-responsive dextrin nanogels delivering doxorubicin on colorectal cancer, Carbohydr. Polym. 126 (2015) 222-230, https://doi.org/10.1016/j carbpol.2015.03.018.

[161] C. Hang, Y. Zou, Y. Zhong, Z. Zhong, F. Meng, NIR and UV-responsive degradable hyaluronic acid nanogels for CD44-targeted and remotely triggered intracellular doxorubicin delivery, Colloids Surf. B: Biointerfaces 158 (2017) 547-555, https:// doi.org/10.1016/j.colsurfb.2017.07.041.

[162] B. Twaites, C. de las Heras Alarcón, C. Alexander, Synthetic polymers as drugs and therapeutics, J. Mater. Chem. 15 (2005) 441-455, https://doi.org/10.1039/ B410799N.

[163] R. Duncan, The dawning era of polymer therapeutics, Nat. Rev. Drug Discov. 2 (2003) 347-360, https://doi.org/10.1038/nrd1088.

[164] R. Duncan, Polymer conjugates as anticancer nanomedicines, Nat. Rev. Cancer 6 (2006) 688-701, https://doi.org/10.1038/nrc1958.

[165] S.C. Owen, D.P.Y. Chan, M.S. Shoichet, Polymeric micelle stability, Nano Today 7 (2012) 53-65, https://doi.org/10.1016/j.nantod.2012.01.002.

[166] G.S. Kwon, T. Okano, Polymeric micelles as new drug carriers, Adv. Drug Deliv. Rev. 21 (1996) 107-116, https://doi.org/10.1016/S0169-409X(96)00401-2.

[167] U. Kedar, P. Phutane, S. Shidhaye, V. Kadam, Advances in polymeric micelles for drug delivery and tumor targeting, Nanomedicine 6 (2010) 714-729, https://doi org/10.1016/j.nano.2010.05.005.

[168] E. Rideau, R. Dimova, P. Schwille, F.R. Wurm, K. Landfester, Liposomes and polymersomes: a comparative review towards cell mimicking, Chem. Soc. Rev. 47 (2018) 8572-8610, https://doi.org/10.1039/C8CS00162F.

[169] J. Ko, K. Park, Y.-S. Kim, M.S. Kim, J.K. Han, K. Kim, R.-W. Park, I.-S. Kim, H.K. Song, D.S. Lee, I.C. Kwon, Tumoral acidic extracellular $\mathrm{pH}$ targeting of $\mathrm{pH}-$ responsive MPEG-poly( $\beta$-amino ester) block copolymer micelles for cancer therapy, J. Control. Release 123 (2007) 109-115, https://doi.org/10.1016/j jconrel.2007.07.012.

[170] G.Y. Lee, K. Park, S.Y. Kim, Y. Byun, MMPs-specific PEGylated peptide-DOX conjugate micelles that can contain free doxorubicin, Eur. J. Pharm. Biopharm. 67 (2007) 646-654, https://doi.org/10.1016/j.ejpb.2007.03.023.

[171] P. Chytil, T. Etrych, Č. Koňák, M. Šírová, T. Mrkvan, J. Bouček, B. Říhová, K. Ulbrich, New HPMA copolymer-based drug carriers with covalently bound hydrophobic substituents for solid tumour targeting. J. Control. Release 127 (2008) 121-130, https://doi.org/10.1016/j.jconrel.2008.01.007.

[172] F.-Q. Hu, L.-N. Liu, Y.-Z. Du, H. Yuan, Synthesis and antitumor activity of doxorubicin conjugated stearic acid-g-chitosan oligosaccharide polymeric micelles, Biomaterials 30 (2009) 6955-6963, https://doi.org/10.1016/j.biomaterials.2009. 09.008

[173] D. Vetvicka, M. Hruby, O. Hovorka, T. Etrych, M. Vetrik, L. Kovar, M. Kovar, K. Ulbrich, B. Rihova, Biological evaluation of polymeric micelles with covalently bound doxorubicin, Bioconjug. Chem. 20 (2009) 2090-2097, https://doi.org/10. 1021/bc900212k.

[174] X.-B. Xiong, Z. Ma, R. Lai, A. Lavasanifar, The therapeutic response to multifunctional polymeric nano-conjugates in the targeted cellular and subcellular delivery of doxorubicin, Biomaterials 31 (2010) 757-768, https://doi.org/10.1016/ j.biomaterials.2009.09.080.

[175] F. Perche, N.R. Patel, V.P. Torchilin, Accumulation and toxicity of antibody-targeted doxorubicin-loaded PEG-PE micelles in ovarian cancer cell spheroid model, J. Control. Release 164 (2012) 95-102, https://doi.org/10.1016/j.jconrel.2012. 09.003.

[176] X. Hu, R. Wang, J. Yue, S. Liu, Z. Xie, X. Jing, Targeting and anti-tumor effect of folic acid-labeled polymer-Doxorubicin conjugates with $\mathrm{pH}$-sensitive hydrazone linker, J. Mater. Chem. 22 (2012) 13303-13310, https://doi.org/10.1039/ 
c2jm31130e.

[177] L. Qiu, M. Zhu, K. Gong, H. Peng, L. Ge, L. Zhao, J. Chen, pH-triggered degradable polymeric micelles for targeted anti-tumor drug delivery, Mater. Sci. Eng. C 78 (2017) 912-922, https://doi.org/10.1016/j.msec.2017.04.137.

[178] Z.S. Liao, S.Y. Huang, J.J. Huang, J.K. Chen, A.W. Lee, J.Y. Lai, D.J. Lee, C.C. Cheng, Self-assembled pH-responsive polymeric micelles for highly efficient, noncytotoxic delivery of doxorubicin chemotherapy to inhibit macrophage activation: in vitro investigation, Biomacromolecules 19 (2018) 2772-2781, https:// doi.org/10.1021/acs.biomac.8b00380.

[179] T. Yang, Y. Lan, M. Cao, X. Ma, A. Cao, Y. Sun, J. Yang, L. Li, Y. Liu, Glycyrrhetinic acid-conjugated polymeric prodrug micelles co-delivered with doxorubicin as combination therapy treatment for liver cancer, Colloids Surf. B: Biointerfaces 175 (2019) 106-115, https://doi.org/10.1016/j.colsurfb.2018.11.082.

[180] K.K. Upadhyay, A.N. Bhatt, A.K. Mishra, B.S. Dwarakanath, S. Jain, C. Schatz, J.F. Le Meins, A. Farooque, G. Chandraiah, A.K. Jain, A. Misra, S. Lecommandoux, The intracellular drug delivery and anti tumor activity of doxorubicin loaded poly ( $\gamma$-benzyl l-glutamate)-b-hyaluronan polymersomes, Biomaterials 31 (2010) 2882-2892, https://doi.org/10.1016/j.biomaterials.2009.12.043.

[181] S.V. Lale, A. Kumar, S. Prasad, A.C. Bharti, V. Koul, Folic acid and trastuzumab functionalized redox responsive polymersomes for intracellular doxorubicin delivery in breast cancer, Biomacromolecules 16 (2015) 1736-1752, https://doi. org/10.1021/acs.biomac.5b00244.

[182] M. Alibolandi, K. Abnous, F. Sadeghi, H. Hosseinkhani, M. Ramezani, F. Hadizadeh, Folate receptor-targeted multimodal polymersomes for delivery of quantum dots and doxorubicin to breast adenocarcinoma: In vitro and in vivo evaluation, Int. J. Pharm. 500 (2016) 162-178, https://doi.org/10.1016/j. ijpharm.2016.01.040.

[183] A.D. Bangham, A correlation between surface charge and coagulant action of phospholipids, Nature 192 (1961) 1197-1198, https://doi.org/10.1038/ $1921197 \mathrm{a} 0$.

[184] A.D. Bangham, R.W. Horne, Negative staining of phospholipids and their structural modification by surface-active agents as observed in the electron microscope, J. Mol. Biol. 8 (1964) 660-668, https://doi.org/10.1016/S0022-2836(64) 80115-7.

[185] T.P. Hoar, J.H. Schulman, Transparent water-in-oil dispersions: the olepathic hydro-micelle, Nature 152 (1943) 102-103, https://doi.org/10.1038/152102a0.

[186] R. Duncan, R. Gaspar, Nanomedicine(s) under the microscope, Mol. Pharm. 8 (2011) 2101-2141, https://doi.org/10.1021/mp200394t.

[187] W. Mehnert, K. Mäder, Solid lipid nanoparticles: production, characterization and applications, Adv. Drug Deliv. Rev. 64 (2012) 83-101, https://doi.org/10.1016/j. addr.2012.09.021.

[188] J. Li, X. Wang, T. Zhang, C. Wang, Z. Huang, X. Luo, Y. Deng, A review on phospholipids and their main applications in drug delivery systems, Asian J. Pharm. Sci. 10 (2014) 81-98, https://doi.org/10.1016/j.ajps.2014.09.004.

[189] G. Verma, P.A. Hassan, Self assembled materials: design strategies and drug delivery perspectives, Phys. Chem. Chem. Phys. 15 (2013) 17016-17028, https:// doi.org/10.1039/c3cp51207j.

[190] C. Kirby, J. Clarke, G. Gregoriadis, Effect of the cholesterol content of small unilamellar liposomes on their stability in vivo and in vitro, Biochem. J. 186 (1980) 591-598, https://doi.org/10.1042/bj1860591.

[191] W.J.M. Mulder, G.J. Strijkers, G.A.F. Van Tilborg, D.P. Cormode, Z.A. Fayad, K. Nicolay, Nanoparticulate assemblies of amphiphiles and diagnostically active materials for multimodality imaging, Acc. Chem. Res. 42 (2009) 904-914, https:// doi.org/10.1021/ar800223c.

[192] J. L. Arias, B. Clares, M. E. Morales, V. Gallardo, M. A. Ruiz, Lipid-based drug delivery systems for cancer treatment, Curr. Drug Targets 12 (2011) 1151-1165, https://doi.org/10.2174/138945011795906570.

[193] Y. Han, Y. Zhang, D. Li, Y. Chen, J. Sun, F. Kong, Transferrin-modified nanostructured lipid carriers as multifunctional nanomedicine for codelivery of DNA and doxorubicin, Int. J. Nanomedicine 9 (2014) 4107-4116, https://doi.org/10 2147/IJN.S67770.

[194] A. Jain, P. Kesharwani, N.K. Garg, A. Jain, S.A. Jain, A.K. Jain, P. Nirbhavane, R. Ghanghoria, R.K. Tyagi, O.P. Katare, Galactose engineered solid lipid nanoparticles for targeted delivery of doxorubicin, Colloids Surf. B: Biointerfaces 134 (2015) 47-58, https://doi.org/10.1016/j.colsurfb.2015.06.027.

[195] A. Tupal, M. Sabzichi, F. Ramezani, M. Kouhsoltani, H. Hamishehkar, Dermal delivery of doxorubicin-loaded solid lipid nanoparticles for the treatment of skin cancer, J. Microencapsul. 33 (2016) 372-380, https://doi.org/10.1080/ 02652048.2016.1200150.

[196] Y. Wang, L. Chen, Y. Ding, W. Yan, Oxidized phospholipid based pH sensitive micelles for delivery of anthracyclines to resistant leukemia cells in vitro, Int. J. Pharm. 422 (2012) 409-417, https://doi.org/10.1016/j.ijpharm.2011.10.029.

[197] H. Hatakeyama, H. Akita, E. Ishida, K. Hashimoto, H. Kobayashi, T. Aoki, J. Yasuda, K. Obata, H. Kikuchi, T. Ishida, H. Kiwada, H. Harashima, Tumor targeting of doxorubicin by anti-MT1-MMP antibody-modified PEG liposomes, Int. J. Pharm. 342 (2007) 194-200, https://doi.org/10.1016/j.ijpharm.2007.04.037.

[198] Q. Li, Q. Tang, P. Zhang, Z. Wang, T. Zhao, J. Zhou, H. Li, Q. Ding, W. Li, F. Hu, Y. Du, H. Yuan, S. Chen, J. Gao, J. Zhan, J. You, Human epidermal growth factor receptor-2 antibodies enhance the specificity and anticancer activity of lightsensitive doxorubicin-labeled liposomes, Biomaterials 57 (2015) 1-11, https:// doi.org/10.1016/j.biomaterials.2015.04.009.

[199] A. Haeri, S. Zalba, T.L.M. ten Hagen, S. Dadashzadeh, G.A. Koning, EGFR targeted thermosensitive liposomes: a novel multifunctional platform for simultaneous tumor targeted and stimulus responsive drug delivery, Colloids Surf. B: Biointerfaces 146 (2016) 657-669, https://doi.org/10.1016/j.colsurfb.2016.06. 012
[200] M. Peller, L. Willerding, S. Limmer, M. Hossann, O. Dietrich, M. Ingrisch, R. Sroka, L.H. Lindner, Surrogate MRI markers for hyperthermia-induced release of doxorubicin from thermosensitive liposomes in tumors, J. Control. Release 237 (2016) 138-146, https://doi.org/10.1016/j.jconrel.2016.06.035.

[201] A. Sesarman, L. Tefas, B. Sylvester, E. Licarete, V. Rauca, L. Luput, L. Patras, M. Banciu, A. Porfire, Anti-angiogenic and anti-inflammatory effects of long-circulating liposomes co-encapsulating curcumin and doxorubicin on C26 murine colon cancer cells, Pharmacol. Rep. 70 (2017) 331-339, https://doi.org/10.1016/ j.pharep.2017.10.004.

[202] K. Plourde, R.M. Derbali, A. Desrosiers, C. Dubath, A. Vallée-Bélisle, J. Leblond, Aptamer-based liposomes improve specific drug loading and release, J. Control. Release 251 (2017) 82-91, https://doi.org/10.1016/j.jconrel.2017.02.026.

[203] Q. Xie, W. Deng, X. Yuan, H. Wang, Z. Ma, B. Wu, X. Zhang, Selenium-functionalized liposomes for systemic delivery of doxorubicin with enhanced pharmacokinetics and anticancer effect, Eur. J. Pharm. Biopharm. 122 (2018) 87-95, https://doi.org/10.1016/j.ejpb.2017.10.010.

[204] J. Nam, N. Won, J. Bang, H. Jin, J. Park, S. Jung, S. Jung, Y. Park, S. Kim, Surface engineering of inorganic nanoparticles for imaging and therapy, Adv. Drug Deliv. Rev. 65 (2013) 622-648, https://doi.org/10.1016/j.addr.2012.08.015.

[205] D. Chen, N.A. Monteiro-Riviere, L.W. Zhang, Intracellular imaging of quantum dots, gold, and iron oxide nanoparticles with associated endocytic pathways, Wiley Interdiscip. Rev. Nanomed. Nanobiotechnol. 9 (2017), https://doi.org/10. 1002/wnan.1419.

[206] M. Yamada, M. Foote, T.W. Prow, Therapeutic gold, silver, and platinum nanoparticles, Wiley Interdiscip. Rev. Nanomed. Nanobiotechnol. 7 (2015) 428-445, https://doi.org/10.1002/wnan.1322.

[207] V.I. Shubayev, T.R. Pisanic, S. Jin, Magnetic nanoparticles for theragnostics, Adv. Drug Deliv. Rev. 61 (2009) 467-477, https://doi.org/10.1016/j.addr.2009.03. 007.

[208] S. Laurent, D. Forge, M. Port, A. Roch, C. Robic, L. Vander Elst, R.N. Muller, Magnetic iron oxide nanoparticles: synthesis, stabilization, vectorization, physicochemical characterizations and biological applications, Chem. Rev. 108 (2008) 2064-2110, https://doi.org/10.1021/cr068445e.

[209] A.K. Gupta, M. Gupta, Synthesis and surface engineering of iron oxide nanoparticles for biomedical applications, Biomaterials 26 (2005) 3995-4021, https:// doi.org/10.1016/j.biomaterials.2004.10.012.

[210] M.-C. Daniel, D. Astruc, Gold nanoparticles: assembly, supramolecular chemistry, quantum-size-related properties, and applications toward biology, catalysis, and nanotechnology, Chem. Rev. 104 (2004) 293-346, https://doi.org/10.1021/ cr030698+.

[211] W. Haiss, N.T.K. Thanh, J. Aveyard, D.G. Fernig, Determination of size and concentration of gold nanoparticles from UV - Vis spectra, Anal. Chem. 79 (2007) 4215-4221, https://doi.org/10.1021/ac0702084.

[212] X.F. Zhang, Z.G. Liu, W. Shen, S. Gurunathan, Silver nanoparticles: synthesis, characterization, properties, applications, and therapeutic approaches, Int. J. Mol. Sci. 17 (2016) 1534-1568, https://doi.org/10.3390/ijms17091534.

[213] M. Ahamed, M.S. AlSalhi, M.K.J. Siddiqui, Silver nanoparticle applications and human health, Clin. Chim. Acta 411 (2010) 1841-1848, https://doi.org/10.1016/ j.cca.2010.08.016.

[214] S. Chernousova, M. Epple, Silver as antibacterial agent: ion, nanoparticle, and metal, Angew. Chem. Int. Ed. 52 (2013) 1636-1653, https://doi.org/10.1002/ anie.201205923.

[215] B. Gaihre, M.S. Khil, D.R. Lee, H.Y. Kim, Gelatin-coated magnetic iron oxide nanoparticles as carrier system: drug loading and in vitro drug release study, Int. J. Pharm. 365 (2009) 180-189, https://doi.org/10.1016/j.ijpharm.2008.08.020.

[216] S. Purushotham, P.E.J. Chang, H. Rumpel, I.H.C. Kee, R.T.H. Ng, P.K.H. Chow, C.K. Tan, R.V. Ramanujan, Thermoresponsive core-shell magnetic nanoparticles for combined modalities of cancer therapy, Nanotechnology 20 (2009) 305101, https://doi.org/10.1088/0957-4484/20/30/305101.

[217] S. Kayal, R.V. Ramanujan, Doxorubicin loaded PVA coated iron oxide nanoparticles for targeted drug delivery, Mater. Sci. Eng. C 30 (2010) 484-490, https:// doi.org/10.1016/j.msec.2010.01.006.

[218] J.H. Maeng, D.-H. Lee, K.H. Jung, Y.-H. Bae, I.-S. Park, S. Jeong, Y.-S. Jeon, C.K. Shim, W. Kim, J. Kim, J. Lee, Y.-M. Lee, J.-H. Kim, W.-H. Kim, S.-S. Hong, Multifunctional doxorubicin loaded superparamagnetic iron oxide nanoparticles for chemotherapy and magnetic resonance imaging in liver cancer, Biomaterials 31 (2010) 4995-5006, https://doi.org/10.1016/j.biomaterials.2010.02.068.

[219] Y. Liu, K. Yang, L. Cheng, J. Zhu, X. Ma, H. Xu, Y. Li, L. Guo, H. Gu, Z. Liu, PEGylated FePt@Fe2O3 core-shell magnetic nanoparticles: Potential theranostic applications and in vivo toxicity studies, Nanomedicine 9 (2013) 1077-1088, https://doi.org/10.1016/j.nano.2013.02.010.

[220] M. Hałupka-Bryl, K. Asai, S. Thangavel, M. Bednarowicz, R. Krzyminiewski, Y. Nagasaki, Synthesis and in vitro and in vivo evaluations of poly(ethylene glycol)-block-poly(4-vinylbenzylphosphonate) magnetic nanoparticles containing doxorubicin as a potential targeted drug delivery system, Colloids Surf. B: Biointerfaces 118 (2014) 140-147, https://doi.org/10.1016/j.colsurfb.2014.03. 025.

[221] A. Pourjavadi, Z.M. Tehrani, N. Mahmoudi, The effect of protein corona on doxorubicin release from the magnetic mesoporous silica nanoparticles with polyethylene glycol coating, J. Nanopart. Res. 17 (2015) 197, , https://doi.org/10. 1007/s11051-015-3008-3.

[222] N. Abbasi Aval, J. Pirayesh Islamian, M. Hatamian, M. Arabfirouzjaei, J. Javadpour, M.-R. Rashidi, Doxorubicin loaded large-pore mesoporous hydroxyapatite coated superparamagnetic $\mathrm{Fe} 3 \mathrm{O} 4$ nanoparticles for cancer treatment, Int. J. Pharm. 509 (2016) 159-167, https://doi.org/10.1016/j.ijpharm.2016.05. 046. 
[223] M. Nadeem, M. Ahmad, M.S. Akhtar, A. Shaari, S. Riaz, S. Naseem, M. Masood, M.A. Saeed, Magnetic properties of polyvinyl alcohol and doxorubicine loaded iron oxide nanoparticles for anticancer drug delivery applications, PLoS ONE 11 (2016) 1-12, https://doi.org/10.1371/journal.pone.0158084.

[224] Y. Yang, Q.F. Guo, J.R. Peng, J. Su, X.L. Lu, Y.X. Zhao, Z.Y. Qian, Doxorubicinconjugated heparin-coated superparamagnetic iron oxide nanoparticles for combined anticancer drug delivery and magnetic resonance imaging, J. Biomed. Nanotechnol. 12 (2016) 1963-1974, https://doi.org/10.1166/jbn.2016.2298.

[225] N.V. Mdlovu, F.A. Mavuso, K.S. Lin, T.W. Chang, Y. Chen, S.S.S. Wang, C.M. Wu, N.B. Mdlovu, Y.S. Lin, Iron oxide-pluronic F127 polymer nanocomposites as carriers for a doxorubicin drug delivery system, Colloids Surfaces A Physicochem. Eng. Asp. 562 (2019) 361-369, https://doi.org/10.1016/j.colsurfa.2018.11.052.

[226] M. Prabaharan, J.J. Grailer, S. Pilla, D.A. Steeber, S. Gong, Gold nanoparticles with a monolayer of doxorubicin-conjugated amphiphilic block copolymer for tumortargeted drug delivery, Biomaterials 30 (2009) 6065-6075, https://doi.org/10. 1016/j.biomaterials.2009.07.048.

[227] H. Chen, S. Li, B. Li, X. Ren, S. Li, D.M. Mahounga, S. Cui, Y. Gu, S. Achilefu, Folate-modified gold nanoclusters as near-infrared fluorescent probes for tumor imaging and therapy, Nanoscale 4 (2012) 6050-6064, https://doi.org/10.1039/ c2nr31616a.

[228] W.-H. Chen, X.-D. Xu, H.-Z. Jia, Q. Lei, G.-F. Luo, S.-X. Cheng, R.-X. Zhuo, X. Z. Zhang, Therapeutic nanomedicine based on dual-intelligent functionalized gold nanoparticles for cancer imaging and therapy in vivo, Biomaterials 34 (2013) 8798-8807, https://doi.org/10.1016/j.biomaterials.2013.07.084.

[229] T.-M. Sun, Y.-C. Wang, F. Wang, J.-Z. Du, C.-Q. Mao, C.-Y. Sun, R.-Z. Tang, Y. Liu, J. Zhu, Y.-H. Zhu, X.-Z. Yang, J. Wang, Cancer stem cell therapy using doxorubicin conjugated to gold nanoparticles via hydrazone bonds, Biomaterials 35 (2014) 836-845, https://doi.org/10.1016/j.biomaterials.2013.10.011.

[230] A. Topete, M. Alatorre-Meda, P. Iglesias, E.M. Villar-Alvarez, S. Barbosa, J.A. Costoya, P. Taboada, V. Mosquera, Fluorescent drug-loaded, polymeric-based, branched gold nanoshells for localized multimodal therapy and imaging of tumoral cells, ACS Nano 8 (2014) 2725-2738, https://doi.org/10.1021/nn406425h.

[231] N.S. Elbialy, M.M. Fathy, W.M. Khalil, Doxorubicin loaded magnetic gold nanoparticles for in vivo targeted drug delivery, Int. J. Pharm. 490 (2015) 190-199, https://doi.org/10.1016/j.ijpharm.2015.05.032.

[232] S. Jeon, H. Ko, N. Vijayakameswara Rao, H.Y. Yoon, D.G. You, H.S. Han, W. Um, G. Saravanakumar, J.H. Park, A versatile gold cross-linked nanoparticle based on triblock copolymer as the carrier of doxorubicin, R. Soc. Chem. Adv. 5 (2015) 70352-70360, https://doi.org/10.1039/C5RA14044G.

[233] X. Zhang, J.G. Teodoro, J.L. Nadeau, Intratumoral gold-doxorubicin is effective in treating melanoma in mice, Nanomedicine 11 (2015) 1365-1375, https://doi.org/ 10.1016/j.nano.2015.04.001.

[234] C.-S. Lee, H. Kim, J. Yu, S.H. Yu, S. Ban, S. Oh, D. Jeong, J. Im, M.J. Baek, T.H. Kim, Doxorubicin-loaded oligonucleotide conjugated gold nanoparticles: a promising in vivo drug delivery system for colorectal cancer therapy, Eur. J. Med. Chem. 142 (2017) 416-423, https://doi.org/10.1016/j.ejmech.2017.08.063.

[235] V. Ramalingam, K. Varunkumar, V. Ravikumar, R. Rajaram, Target delivery of doxorubicin tethered with PVP stabilized gold nanoparticles for effective treatment of lung cancer, Sci. Rep. 8 (2018) 1-12, https://doi.org/10.1038/s41598018-22172-5.

[236] G. Hou, J. Qian, W. Xu, T. Sun, Y. Wang, J. Wang, L. Ji, A. Suo, A novel pHsensitive targeting polysaccharide-gold nanorod conjugate for combined photothermal-chemotherapy of breast cancer, Carbohydr. Polym. 212 (2019) 334-344, https://doi.org/10.1016/j.carbpol.2019.02.045.

[237] C. Cha, S.R. Shin, N. Annabi, M.R. Dokmeci, A. Khademhosseini, Carbon-based nanomaterials: multifunctional materials for biomedical engineering, ACS Nano 7 (2013) 2891-2897, https://doi.org/10.1021/nn401196a.

[238] K.S. Novoselov, V.I. Fal'ko, L. Colombo, P.R. Gellert, M.G. Schwab, K. Kim, A roadmap for graphene, Nature 490 (2013) 192-200, https://doi.org/10.1038/ nature 11458

[239] K.D. Patel, R.K. Singh, H.W. Kim, Carbon-based nanomaterials as an emerging platform for theranostics, Mater. Horizons. 6 (2019) 434-469, https://doi.org/10. 1039/c8mh00966j.

[240] T. Murakami, J. Fan, M. Yudasaka, S. Iijima, K. Shiba, Solubilization of single-wall carbon nanohorns using a PEG-doxorubicin conjugate, Mol. Pharm. 3 (2006) 407-414, https://doi.org/10.1021/mp060027a.

[241] H. Ali-Boucetta, K.T. Al-Jamal, D. McCarthy, M. Prato, A. Bianco, K. Kostarelos, Multiwalled carbon nanotube-doxorubicin supramolecular complexes for cancer therapeutics, Chem. Commun. 0 (2008) 459-461, https://doi.org/10.1039/ B712350G.

[242] X. Zhang, L. Meng, Q. Lu, Z. Fei, P.J. Dyson, Targeted delivery and controlled release of doxorubicin to cancer cells using modified single wall carbon nanotubes, Biomaterials 30 (2009) 6041-6047, https://doi.org/10.1016/j.biomaterials. 2009. 07.025 .

[243] Z. Liu, A.C. Fan, K. Rakhra, S. Sherlock, A. Goodwin, X. Chen, Q. Yang, D.W. Felsher, H. Dai, Supramolecular stacking of doxorubicin on carbon nanotubes for in vivo cancer therapy, Angew. Chem. Int. Ed. 48 (2009) 7668-7672, https://doi.org/10.1002/anie.200902612.

[244] Y.-J. Gu, J. Cheng, J. Jin, S.H. Cheng, W.-T. Wong, Development and evaluation of pH-responsive single-walled carbon nanotube-doxorubicin complexes in cancer cells, Int. J. Nanomedicine 6 (2011) 2889-2898, https://doi.org/10.2147/IJN. S25162.

[245] R. Li, R. Wu, L. Zhao, Z. Hu, S. Guo, X. Pan, H. Zou, Folate and iron difunctionalized multiwall carbon nanotubes as dual-targeted drug nanocarrier to cancer cells, Carbon N. Y. 49 (2011) 1797-1805, https://doi.org/10.1016/j.carbon.2011. 01.003 .
[246] N.M. Dinan, F. Atyabi, M.-R. Rouini, M. Amini, A.-A. Golabchifar, R. Dinarvand, Doxorubicin loaded folate-targeted carbon nanotubes: Preparation, cellular internalization, in vitro cytotoxicity and disposition kinetic study in the isolated perfused rat liver, Mater. Sci. Eng. C 39 (2014) 47-55, https://doi.org/10.1016/j. msec.2014.01.055.

[247] X. Qi, Y. Rui, Y. Fan, H. Chen, N. Ma, Z. Wu, Galactosylated chitosan-grafted multiwall carbon nanotubes for $\mathrm{pH}$-dependent sustained release and hepatic tumor-targeted delivery of doxorubicin in vivo, Colloids Surf. B: Biointerfaces 133 (2015) 314-322, https://doi.org/10.1016/j.colsurfb.2015.06.003.

[248] S. Pandey, M. Thakur, A. Mewada, D. Anjarlekar, N. Mishra, M. Sharon, Carbon dots functionalized gold nanorod mediated delivery of doxorubicin: tri-functional nano-worms for drug delivery, photothermal therapy and bioimaging, J. Mater. Chem. B 1 (2013) 4972, https://doi.org/10.1039/c3tb20761g.

[249] J.-H. Liu, L. Cao, P.G. Luo, S.-T. Yang, F. Lu, H. Wang, M.J. Meziani, S.A. Haque, Y. Liu, S. Lacher, Y.-P. Sun, Fullerene-conjugated doxorubicin in cells, ACS Appl Mater. Interfaces 2 (2010) 1384-1389, https://doi.org/10.1021/am100037y.

[250] I. Blazkova, H. Viet Nguyen, M. Kominkova, R. Konecna, D. Chudobova, L. Krejcova, P. Kopel, D. Hynek, O. Zitka, M. Beklova, V. Adam, R. Kizek, Fullerene as a transporter for doxorubicin investigated by analytical methods and in vivo imaging, Electrophoresis 35 (2014) 1040-1049, https://doi.org/10.1002/elps. 201300393.

[251] J. Shi, Y. Liu, L. Wang, J. Gao, J. Zhang, X. Yu, R. Ma, R. Liu, Z. Zhang, A tumoral acidic pH-responsive drug delivery system based on a novel photosensitizer (fullerene) for in vitro and in vivo chemo-photodynamic therapy, Acta Biomater. 10 (2014) 1280-1291, https://doi.org/10.1016/j.actbio.2013.10.037.

[252] G.E. Magoulas, M. Bantzi, D. Messari, E. Voulgari, C. Gialeli, D. Barbouri, A. Giannis, N.K. Karamanos, D. Papaioannou, K. Avgoustakis, Synthesis and evaluation of anticancer activity in cells of novel stoichiometric pegylated fullerenedoxorubicin conjugates, Pharm. Res. 32 (2015) 1676-1693, https://doi.org/10. 1007/s11095-014-1566-1.

[253] A. Mewada, S. Pandey, M. Thakur, D. Jadhav, M. Sharon, Swarming carbon dots for folic acid mediated delivery of doxorubicin and biological imaging, J. Mater. Chem. B 2 (2014) 698-705, https://doi.org/10.1039/C3TB21436B.

[254] M. Zhang, P. Yuan, N. Zhou, Y. Su, M. Shao, C. Chi, pH-Sensitive N-doped carbon dots-heparin and doxorubicin drug delivery system: preparation and anticancer research, R. Soc. Chem. Adv. 7 (2017) 9347-9356, https://doi.org/10.1039/ C6RA28345D.

[255] T. Sun, M. Zheng, Z. Xie, X. Jing, Supramolecular hybrids of carbon dots with doxorubicin: synthesis, stability and cellular trafficking, Mater. Chem. Front. 1 (2017) 354-360, https://doi.org/10.1039/C6QM00042H.

[256] N. Gao, W. Yang, H. Nie, Y. Gong, J. Jing, L. Gao, X. Zhang, Turn-on theranostic fluorescent nanoprobe by electrostatic self-assembly of carbon dots with doxorubicin for targeted cancer cell imaging, in vivo hyaluronidase analysis, and targeted drug delivery, Biosens. Bioelectron. 96 (2017) 300-307, https://doi.org/10 1016/j.bios.2017.05.019.

[257] T. Kong, L. Hao, Y. Wei, X. Cai, B. Zhu, Doxorubicin conjugated carbon dots as a drug delivery system for human breast cancer therapy, Cell Prolif. 51 (2018) 1-9, https://doi.org/10.1111/cpr.12488.

[258] F. Wu, M. Zhang, H. Lu, D. Liang, Y. Huang, Y. Xia, Y. Hu, S. Hu, J. Wang, X. Yi, J. Zhang, Triple stimuli-responsive magnetic hollow porous carbon-based nanodrug delivery system for magnetic resonance imaging-guided synergistic photothermal/chemotherapy of cancer, ACS Appl. Mater. Interfaces 10 (2018) 21939-21949, https://doi.org/10.1021/acsami.8b07213.

[259] R. Wu, J. Liu, D. Chen, J. Pan, Carbon nanodots modified with catechol-borane moieties for $\mathrm{pH}$-stimulated doxorubicin delivery: Toward nuclear targeting, ACS Appl. Nano Mater. 2 (2019) 4333-4341, https://doi.org/10.1021/acsanm. $9 \mathrm{~b} 00779$.

[260] H. Tomás, C.S. Alves, J. Rodrigues, Laponite ${ }^{\oplus}$ : a key nanoplatform for biomedica applications? Nanomedicine 14 (2018) 2407-2420, https://doi.org/10.1016/j. nano.2017.04.016.

[261] BYK Additives \& Instruments, Laponite: performance additives, LAPONITE Perform. Addit. (2014) 1-24 https://www.byk.com/.../BYK B-RI21_LAPONITE EN.pdf.

[262] D. Bonn, H. Kellay, H. Tanaka, G. Wegdam, J. Meunier, Laponite: what is the difference between a gel and a glass? Langmuir 15 (1999) 7534-7536, https://doi org/10.1021/la990167+.

[263] D.W. Thompson, J.T. Butterworth, The nature of laponite and its aqueous dispersions, J. Colloid Interface Sci. 151 (1992) 236-243, https://doi.org/10.1016/ 0021-9797(92)90254-J.

[264] S. Wang, Y. Wu, R. Guo, Y. Huang, S. Wen, M. Shen, J. Wang, X. Shi, Laponite nanodisks as an efficient platform for doxorubicin delivery to cancer cells, Langmuir 29 (2013) 5030-5036, https://doi.org/10.1021/la4001363.

[265] K. Li, S. Wang, S. Wen, Y. Tang, J. Li, X. Shi, Q. Zhao, Enhanced in vivo antitumor efficacy of doxorubicin encapsulated within laponite nanodisks, ACS Appl. Mater. Interfaces 6 (2014) 12328-12334, https://doi.org/10.1021/am502094a.

[266] P. Yousefpour, A. Chilkoti, Co-opting biology to deliver drugs, Biotechnol. Bioeng. 111 (2014) 1699-1716, https://doi.org/10.1002/bit.25307.

[267] S. Dreis, F. Rothweiler, M. Michaelis, J. Cinatl, J. Kreuter, K. Langer, Preparation, characterisation and maintenance of drug efficacy of doxorubicin-loaded human serum albumin (HSA) nanoparticles, Int. J. Pharm. 341 (2007) 207-214, https:// doi.org/10.1016/j.ijpharm.2007.03.036.

[268] S. Wagner, F. Rothweiler, M.G. Anhorn, D. Sauer, I. Riemann, E.C. Weiss, A. Katsen-Globa, M. Michaelis, J. Cinatl, D. Schwartz, J. Kreuter, H. von Briesen, K. Langer, Enhanced drug targeting by attachment of an anti $\alpha \mathrm{v}$ integrin antibody to doxorubicin loaded human serum albumin nanoparticles, Biomaterials 31 (2010) 2388-2398, https://doi.org/10.1016/j.biomaterials.2009.11.093. 
[269] R. Xu, M. Fisher, R.L. Juliano, Targeted albumin-based nanoparticles for delivery of amphipathic drugs, Bioconjug. Chem. 22 (2011) 870-878, https://doi.org/10. 1021/bc1002295.

[270] S. Bae, K. Ma, T.H. Kim, E.S. Lee, K.T. Oh, E.S. Park, K.C. Lee, Y.S. Youn, Doxorubicin-loaded human serum albumin nanoparticles surface-modified with TNF-related apoptosis-inducing ligand and transferrin for targeting multiple tumor types, Biomaterials 33 (2012) 1536-1546, https://doi.org/10.1016/j. biomaterials.2011.10.050

[271] S. Ichimizu, H. Watanabe, H. Maeda, K. Hamasaki, Y. Nakamura, V.T.G. Chuang, R. Kinoshita, K. Nishida, R. Tanaka, Y. Enoki, Y. Ishima, A. Kuniyasu, Y. Kobashigawa, H. Morioka, S. Futaki, M. Otagiri, T. Maruyama, Design and tuning of a cell-penetrating albumin derivative as a versatile nanovehicle for intracellular drug delivery, J. Control. Release 277 (2018) 23-34, https://doi.org/ 10.1016/j.jconrel.2018.02.037.

[272] K. Kimura, K. Yamasaki, K. Nishi, K. Taguchi, M. Otagiri, Investigation of antitumor effect of doxorubicin-loaded human serum albumin nanoparticles prepared by a desolvation technique, Cancer Chemother. Pharmacol. 83 (2019) 1113-1120, https://doi.org/10.1007/s00280-019-03832-3.

[273] S.C. Jang, O.Y. Kim, C.M. Yoon, D.S. Choi, T.Y. Roh, J. Park, J. Nilsson, J. Lötvall, Y.K. Kim, Y.S. Gho, Bioinspired exosome-mimetic nanovesicles for targeted delivery of chemotherapeutics to malignant tumors, ACS Nano 7 (2013) 7698-7710, https://doi.org/10.1021/nn402232g.

[274] Y. Yang, Y. Chen, F. Zhang, Q. Zhao, H. Zhong, Increased anti-tumour activity by exosomes derived from doxorubicin-treated tumour cells via heat stress, Int. J. Hyperth. 31 (2015) 498-506, https://doi.org/10.3109/02656736.2015.1036384.

[275] A. Srivastava, N. Amreddy, A. Babu, J. Panneerselvam, M. Mehta,

R. Muralidharan, A. Chen, Y.D. Zhao, M. Razaq, N. Riedinger, H. Kim, S. Liu, S. Wu, A.B. Abdel-Mageed, A. Munshi, R. Ramesh, Nanosomes carrying doxorubicin exhibit potent anticancer activity against human lung cancer cells, Sci. Rep. 6 (2016) 1-15, https://doi.org/10.1038/srep38541.

[276] W.J. Goh, C.K. Lee, S. Zou, E.C.Y. Woon, B. Czarny, G. Pastorin, Doxorubicinloaded cell-derived nanovesicles: an alternative targeted approach for anti-tumor therapy, Int. J. Nanomedicine 12 (2017) 2759-2767, https://doi.org/10.2147/ IJN.S131786.

[277] O. of the Commissioner, The Drug Development Process, https://www.fda.gov/ ForPatients/Approvals/Drugs/default.htm, (2017), Accessed date: 9 October 2017.

[278] Clinical Studies Explained, https://neurosciences.ucsd.edu/centers/huntingtonsdisease/research/Pages/clinical-observational-trials.aspx, (2018), Accessed date: 27 March 2018.

[279] J.M. Caster, A.N. Patel, T. Zhang, A. Wang, Investigational nanomedicines in 2016: a review of nanotherapeutics currently undergoing clinical trials, Wiley Interdiscip. Rev. Nanomed. Nanobiotechnol. 9 (2017), https://doi.org/10.1002/ wnan.1416.

[280] Y. Min, J.M. Caster, M.J. Eblan, A.Z. Wang, Clinical translation of nanomedicine, Chem. Rev. 115 (2015) 11147-11190, https://doi.org/10.1021/acs.chemrev. 5 b00116.

[281] S. Svenson, Clinical translation of nanomedicines, Curr. Opin. Solid State Mater. Sci. 16 (2012) 287-294, https://doi.org/10.1016/j.cossms.2012.10.001.

[282] H. Havel, G. Finch, P. Strode, M. Wolfgang, S. Zale, I. Bobe, H. Youssoufian, M. Peterson, M. Liu, Nanomedicines: from bench to bedside and beyond, Am. Assoc. Pharm. Sci. J. 18 (2016) 1373-1378, https://doi.org/10.1208/s12248-0169961-7.

[283] S. Hassan, G. Prakasha, A. Bal Ozturk, S. Saghazadeh, M. Farhan Sohail, J. Seo, M. Remzi Dokmeci, Y.S. Zhang, A. Khademhosseini, Evolution and clinical translation of drug delivery nanomaterials, Nano Today 15 (2017) 91-106, https://doi.org/10.1016/j.nantod.2017.06.008.

[284] S. Stewart, H. Jablonowski, F.D. Goebel, K. Arasteh, M. Spittle, A. Rios, D. Aboulafia, J. Galleshaw, B.J. Dezube, Randomized comparative trial of pegylated liposomal doxorubicin versus bleomycin and vincristine in the treatment of AIDS-related Kaposi's sarcoma, J. Clin. Oncol. 16 (1998) 683-691, https://doi. org /10.1200/JCO.1998.16.2.683.

[285] D.W. Northfelt, B.J. Dezube, J.A. Thommes, B.J. Miller, M.A. Fischl, A. FriedmanKien, L.D. Kaplan, C. Du Mond, R.D. Mamelok, D.H. Henry, Pegylated-liposomal doxorubicin versus doxorubicin, bleomycin, and vincristine in the treatment of AIDS-related Kaposi's sarcoma: results of a randomized phase III clinical trial, J. Clin. Oncol. 16 (1998) 2445-2451, https://doi.org/10.1200/JCO.1998.16.7. 2445 .

[286] M.E.R. O'Brien, N. Wigler, M. Inbar, R. Rosso, E. Grischke, A. Santoro, R. Catane, D.G. Kieback, P. Tomczak, S.P. Ackland, F. Orlandi, L. Mellars, L. Alland, C. Tendler, Reduced cardiotoxicity and comparable efficacy in a phase III trial of pegylated liposomal doxorubicin $\mathrm{HCl}\left(\mathrm{CAELYX}^{\mathrm{TM}} /\right.$ Doxil $\left.^{\circledast}\right)$ versus conventional doxorubicin for first-line treatment of metastatic breast cancer, Ann. Oncol. 15 (2004) 440-449, https://doi.org/10.1093/annonc/mdh097.

[287] A.N. Gordon, M. Tonda, S. Sun, W. Rackoff, Long-term survival advantage for women treated with pegylated liposomal doxorubicin compared with topotecan in a phase 3 randomized study of recurrent and refractory epithelial ovarian cancer, Gynecol. Oncol. 95 (2004) 1-8, https://doi.org/10.1016/j.ygyno.2004.07.011.

[288] R.M. Rifkin, S.A. Gregory, A. Mohrbacher, M.A. Hussein, Pegylated liposomal doxorubicin, vincristine, and dexamethasone provide significant reduction in toxicity compared with doxorubicin, vincristine, and dexamethasone in patients with newly diagnosed multiple myeloma: a phase III multicenter randomized trial, Cancer 106 (2006) 848-858, https://doi.org/10.1002/cncr.21662.

[289] G. Batist, G. Ramakrishnan, C.S. Rao, A. Chandrasekharan, J. Gutheil, T. Guthrie, P. Shah, A. Khojasteh, M.K. Nair, K. Hoelzer, K. Tkaczuk, Y.C. Park, L.W. Lee Reduced cardiotoxicity and preserved antitumor eiicacy of liposome-encapsulated doxorubicin and multicenter trial of metastatic breast cancer, J. Clin. Oncol. 19 (2001) 1444-1454, https://doi.org/10.1200/JCO.2001.19.5.1444.

[290] G. Batist, J. Barton, P. Chaikin, C. Swenson, L. Welles, Myocet (liposome-encapsulated doxorubicin citrate): a new approach in breast cancer therapy, Expert. Opin. Pharmacother. 3 (2002) 1739-1751, https://doi.org/10.1517/14656566.3. 12.1739.

[291] L. Harris, G. Batist, R. Belt, D. Rovira, R. Navari, N. Azarnia, L. Welles, E. Winer, Liposome-encapsulated doxorubicin compared with conventional doxorubicin in a randomized multicenter trial as first-line therapy of metastatic breast carcinoma, Cancer 94 (2002) 25-36, https://doi.org/10.1002/cncr.10201.

[292] S. Chan, N. Davidson, E. Juozaityte, F. Erdkamp, A. Pluzanska, N. Azarnia, L.W. Lee, Phase III trial of liposomal doxorubicin and cyclophosphamide compared with epirubicin and cyclophosphamide as first-line therapy for metastatic breast cancer, Ann. Oncol. 15 (2004) 1527-1534, https://doi.org/10.1093/ annonc/mdh393.

[293] Celsion, Study of ThermoDox With Standardized Radiofrequency Ablation (RFA) for Treatment of Hepatocellular Carcinoma (HCC) (OPTIMA), https:// Clinicaltrials.Gov/Ct2/Show/Study/NCT02112656. (2014) NCT02112656.

[294] Celsion, Phase 3 Study of ThermoDox With Radiofrequency Ablation (RFA) in Treatment of Hepatocellular Carcinoma (HCC), ClinicalTrials.Gov, 2014, https: clinicaltrials.gov/ct2/show/NCT00617981, Accessed date: 17 June 2019.

[295] T.M. Zagar, Z. Vujaskovic, S. Formenti, H. Rugo, F. Muggia, B. O'Connor, R. Myerson, P. Stauffer, I.-C. Hsu, C. Diederich, W. Straube, M.-K. Boss, A. Boico, O. Craciunescu, P. Maccarini, D. Needham, N. Borys, K.L. Blackwell, M.W. Dewhirst, Two phase I dose-escalation/pharmacokinetics studies of low temperature liposomal doxorubicin (LTLD) and mild local hyperthermia in heavily pretreated patients with local regionally recurrent breast cancer, Int. J. Hyperth. 30 (2014) 285-294, https://doi.org/10.3109/02656736.2014.936049.

[296] P.C. Lyon, L.F. Griffiths, J. Lee, D. Chung, R. Carlisle, F. Wu, M.R. Middleton, F.V. Gleeson, C.C. Coussios, Clinical trial protocol for TARDOX: a phase I study to investigate the feasibility of targeted release of lyso-thermosensitive liposomal doxorubicin (ThermoDox ${ }^{\oplus}$ ) using focused ultrasound in patients with liver tumours, J. Ther. Ultrasound. 5 (2017) 28, , https://doi.org/10.1186/s40349-0170104-0.

[297] A. Kim, A Phase I Study of Lyso-thermosensitive Liposomal Doxorubicin and MRHIFU for Pediatric Refractory Solid Tumors, vol. 2015-2020, Clinicaltrials.Gov, 2015, https://clinicaltrials.gov/ct2/show/NCT02536183?term $=02536183 \&$ rank $=1$, Accessed date: 17 April 2019.

[298] UMC Utrecht, Image-Guided Targeted Doxorubicin Delivery with Hyperthermia to Optimize Loco-Regional Control in breast Cancer, https://clinicaltrials.gov/ct2/ show $/$ NCT03749850?term $=03749850 \&$ rank $=1$, (2019) , Accessed date: 17 Apri 2019.

[299] A. Lopez-Pousa, B.B. Nguyen, X.G. del Muro, J.M. Broto, C. Balañá, J. Lavernia, J. Cruz, J. Maurel, R. Andres, C.M. Valverde, J. Fra, J. Martinez-Trufero, J.A. Lopez-Martin, I. Sevilla, R. Cubedo, J. Blay, A phase II study of a new formulation of nonpegylated liposomal doxorubicin (doxorubicin GP-pharm) as firstline treatment in patients with advanced soft-tissue sarcomas (STS) who are age 65 or older: a GEIS trial, J. Clin. Oncol. 29 (2011) 10072, https://doi.org/10.1200/ jco.2011.29.15_suppl.10072.

[300] MM-302 Misses Endpoint in Phase II Hermione Trial - ADC Review ADC Review, https://adcreview.com/news/mm-302-misses-endpoint-phase-ii-hermione-trial/, (2019) , Accessed date: 17 June 2019.

[301] Onxeo Announces Top-Line Results from ReLive Phase III Study of Livatag ${ }^{\circledR}$ in Advanced Hepatocellular Carcinoma, https://www.onxeo.com/onxeo-announcestop-line-results-relive-phase-iii-study-livatag-advanced-hepatocellularcarcinoma/, (2017), Accessed date: 17 June 2019.

[302] Onxeo to host a Conference Call today to comment on the main findings from ReLive Phase III Study of Livatag ${ }^{\circledR}$, https://www.onxeo.com/onxeo-hostconference-call-today-comment-main-findings-relive-phase-iii-study-livatag/, (2019) , Accessed date: 17 June 2019.

[303] L.W. Seymour, D.R. Ferry, D.J. Kerr, D. Rea, M. Whitlock, R. Poyner, C. Boivin, S. Hesslewood, C. Twelves, R. Blackie, A. Schatzlein, D. Jodrell, D. Bissett, H. Calvert, M. Lind, A. Robbins, S. Burtles, R. Duncan, J. Cassidy, Phase II studies of polymer-doxorubicin (PK1, FCE28068) in the treatment of breast, lung and colorectal cancer, Int. J. Oncol. 34 (2009) 1629-1636, https://doi.org/10.3892/ ijo_00000293.

[304] J.W. Valle, J. Lawrance, J. Brewer, A. Clayton, P. Corrie, V. Alakhov, M. Ranson, A phase II, window study of SP1049C as first-line therapy in inoperable metastatic adenocarcinoma of the oesophagus, J. Clin. Oncol. 22 (2004), https://doi.org/10. 1200/jco.2004.22.90140.4195 4195-4195.

[305] Y. Matsumura, T. Hamaguchi, T. Ura, K. Muro, Y. Yamada, Y. Shimada, K. Shirao, T. Okusaka, H. Ueno, M. Ikeda, N. Watanabe, Phase I clinical trial and pharmacokinetic evaluation of NK911, a micelle-encapsulated doxorubicin, Br. J. Cancer 91 (2004) 1775-1781, https://doi.org/10.1038/sj.bjc.6602204.

[306] J.R. Whittle, J.D. Lickliter, H.K. Gan, A.M. Scott, J. Simes, B.J. Solomon, J.A. MacDiarmid, H. Brahmbhatt, M.A. Rosenthal, First in human nanotechnology doxorubicin delivery system to target epidermal growth factor receptors in recurrent glioblastoma, J. Clin. Neurosci. 22 (2015) 1889-1894, https://doi.org/10. 1016/j.jocn.2015.06.005.

[307] G. Sessa, G. Weissmann, Phospholipid spherules (liposomes) as a model for biological membranes, J. Lipid Res. 9 (1968) 310-318.

[308] Y. Barenholz, A. Gabizon, Liposome/Doxorubicin Compositon and Method, 4 (1990), p. 898,735.

[309] N.D. James, R.J. Coker, D. Tomlinson, J.R.W. Harris, M. Gompels, A.J. Pinching, J.S.W. Stewart, Liposomal doxorubicin (Doxil): an effective new treatment for Kaposi's sarcoma in AIDS, Clin. Oncol. 6 (1994) 294-296, https://doi.org/10. 
1016/S0936-6555(05)80269-9.

[310] F.M. Muggia, J.D. Hainsworth, S. Jeffers, P. Miller, S. Groshen, M. Tan, L. Roman, B. Uziely, L. Muderspach, A. Garcia, A. Burnett, F.A. Greco, C.P. Morrow, L.J. Paradiso, L.-J. Liang, Phase II study of liposomal doxorubicin in refractory ovarian cancer: antitumor activity and toxicity modification by liposomal encapsulation, J. Clin. Oncol. 15 (1997) 987-993, https://doi.org/10.1200/JCO. 1997.15.3.987.

[311] Z. Symon, A. Peyser, D. Tzemach, O. Lyass, E. Sucher, E. Shezen, A. Gabizon, Selective delivery of doxorubicin to patients with breast carcinoma metastases by stealth liposomes, Cancer 86 (1999) 72-78, https://doi.org/10.1002/(SICI)10970142(19990701)86:1 < 72::AID-CNCR12> 3.0.CO;2-1.

[312] R.Z. Orlowski, A. Nagler, P. Sonneveld, J. Bladé, R. Hajek, A. Spencer, J. San Miguel, T. Robak, A. Dmoszynska, N. Horvath, I. Spicka, H.J. Sutherland, A.N. Suvorov, S.H. Zhuang, T. Parekh, L. Xiu, Z. Yuan, W. Rackoff, J.L. Harousseau, Randomized phase III study of pegylated liposomal doxorubicin plus bortezomib compared with bortezomib alone in relapsed or refractory multiple myeloma: combination therapy improves time to progression, J. Clin. Oncol. 25 (2007) 3892-3901, https://doi.org/10.1200/JCO.2006.10.5460.

[313] A. Gabizon, H. Shmeeda, Y. Barenholz, Pharmacokinetics of pegylated liposomal doxorubicin: review of animal and human studies, Clin. Pharmacokinet. 42 (2003) 419-436, https://doi.org/10.2165/00003088-200342050-00002.

[314] P.K. Working, A.D. Dayan, Pharmacological-toxicological expert report. CAELYX. (Stealth liposomal doxorubicin HCl), Hum. Exp. Toxicol. 15 (1996) 751-785 http://www.ncbi.nlm.nih.gov/pubmed/8880211.

[315] S.E. Krown, D.W. Northfelt, D. Osoba, J.S. Stewart, Use of liposomal anthracyclines in Kaposi's sarcoma, Semin. Oncol. 31 (2004) 36-52, https://doi.org/10. 1053/j.seminoncol.2004.08.003.

[316] U. Bulbake, S. Doppalapudi, N. Kommineni, W. Khan, Liposomal formulations in clinical use: an updated review, Pharmaceutics 9 (2017) 1-33, https://doi.org/10. 3390/pharmaceutics9020012.

[317] R.C.F. Leonard, S. Williams, A. Tulpule, A.M. Levine, S. Oliveros, Improving the therapeutic index of anthracycline chemotherapy : focus on liposomal doxorubicin (Myocet $^{\mathrm{TM}}$ ), Breast 18 (2009) 218-224, https://doi.org/10.1016/j.breast.2009. 05.004 .

[318] P.M. Kanter, G. Klaich, G.A. Bullard, J.M. King, Z.P. Pavelic, Preclinical toxicology study of liposome encapsulated doxorubicin (TLC D-99) given intraperitoneally to dogs, In Vivo (Brooklyn). 8 (1994) 975-982 http://www.ncbi.nlm.nih.gov/ pubmed/8504212, Accessed date: 6 October 2017.

[319] R. Ginsberg, F. Pilkiewicz, R. Ginsberg, D.E. Brenner, Y. Tung, N. Petrelli, Initial clinical (Phase I) trial of TLC D-99 (doxorubicin encapsulated in liposomes), Cancer Res. 53 (1993) 2796-2802.

[320] R.T. Poon, N. Borys, Lyso-thermosensitive liposomal doxorubicin: an adjuvant to increase the cure rate of radiofrequency ablation in liver cancer, Future Oncol. 7 (2011) 937-945, https://doi.org/10.2217/fon.11.73.

[321] R.T. Poon, N. Borys, Lyso-thermosensitive liposomal doxorubicin: a novel approach to enhance efficacy of thermal ablation of liver cancer, Expert. Opin. Pharmacother. 10 (2009) 333-343, https://doi.org/10.1517/ 14656560802677874.

[322] J. Chen, C.-Q. He, A.-H. Lin, W. Gu, Z.-P. Chen, W. Li, B.-C. Cai, Thermosensitive liposomes with higher phase transition temperature for targeted drug delivery to tumor, Int. J. Pharm. 475 (2014) 408-415, https://doi.org/10.1016/j.ijpharm. 2014.09.009.

[323] B. Kneidl, M. Peller, G. Winter, L.H. Lindner, M. Hossann, Thermosensitive liposomal drug delivery systems: state of the art review, Int. J. Nanomedicine 9 (2014) 4387-4398, https://doi.org/10.2147/IJN.S49297.

[324] A. Puri, Phototriggerable liposomes: current research and future perspectives, Pharmaceutics 6 (2014) 1-25, https://doi.org/10.3390/pharmaceutics6010001.

[325] S.N. Goldberg, I.R. Kamel, J.B. Kruskal, K. Reynolds, W.L. Monsky, K.E. Stuart, M. Ahmed, V. Raptopoulos, Radiofrequency ablation of hepatic tumors: increased tumor destruction with adjuvant liposomal doxorubicin therapy, Am. J. Roentgenol. 179 (2002) 93-101, https://doi.org/10.2214/ajr.179.1.1790093.

[326] M.D. Gray, P.C. Lyon, C. Mannaris, L.K. Folkes, M. Stratford, L. Campo, D.Y.F. Chung, S. Scott, M. Anderson, R. Goldin, R. Carlisle, F. Wu, M.R. Middleton, F.V. Gleeson, C.C. Coussios, Focused ultrasound hyperthermia for targeted drug release from thermosensitive liposomes: results from a phase I trial, Radiology 291 (2019) 232-238, https://doi.org/10.1148/radiol.2018181445.

[327] V. Patravale, P. Dandekar, R. Jain, Clinical trials industrial aspects, Nanoparticulate Drug Deliv, Elsevier, 2012, pp. 191-207, , https://doi.org/10. 1533/9781908818195.191.

[328] P.J. Gaillard, C.C.M. Appeldoorn, R. Dorland, J. Van Kregten, F. Manca, D.J. Vugts, B. Windhorst, G.A.M.S. Van Dongen, H.E. De Vries, D. Maussang, $\mathrm{O}$. Van Tellingen, Pharmacokinetics, brain delivery, and efficacy in brain tumorbearing mice of glutathione pegylated liposomal doxorubicin (2B3-101), PLoS ONE 9 (2014) e82331, , https://doi.org/10.1371/journal.pone.0082331.

[329] J.W. Park, K. Hong, D.B. Kirpotin, D. Papahadjopoulos, C.C. Benz, Immunoliposomes for cancer treatment, Adv. Pharmacol. (1997) 399-435, https://doi.org/10.1016/S1054-3589(08)60146-5.

[330] C. Rochlitz, C. Mamot, Immunoliposomes for Treatment of Cancer, US 2010/ 0239652 A1 (2010).

[331] C. Mamot, D.C. Drummond, U. Greiser, K. Hong, D.B. Kirpotin, J.D. Marks, J.W. Park, Epidermal growth factor receptor (EGFR)-targeted immunoliposomes mediate specific and efficient drug delivery to EGFR- and EGFRvIII-overexpressing tumor cells, Cancer Res. 63 (2003) 3154-3161, https://doi.org/10.1080/ 14733400410001727592

[332] C. Mamot, D.C. Drummond, C.O. Noble, V. Kallab, Z. Guo, K. Hong, D.B. Kirpotin, J.W. Park, Epidermal growth factor receptor-targeted immunoliposomes significantly enhance the efficacy of multiple anticancer drugs in vivo, Cancer Res. 65 (2005) 11631-11638, https://doi.org/10.1158/0008-5472.CAN-05-1093.

[333] C. Mamot, R. Ritschard, A. Wicki, W. Küng, J. Schuller, R. Herrmann, C. Rochlitz, Immunoliposomal delivery of doxorubicin can overcome multidrug resistance mechanisms in EGFR-overexpressing tumor cells, J. Drug Target. 20 (2012) 422-432, https://doi.org/10.3109/1061186X.2012.680960.

[334] Merrimack, Dosage and administration for preventing cardiotoxicity in treatment with ERBB2-targeted immunoliposomes comprising anthracyclin chemotherapeutic agents, US20160038416A1, 2012.

[335] J.W. Park, D.B. Kirpotin, K. Hong, R. Shalaby, Y. Shao, U.B. Nielsen, J.D. Marks, D. Papahadjopoulos, C.C. Benz, Tumor targeting using anti-her2 immunoliposomes, J. Control. Release 74 (2001) 95-113, https://doi.org/10.1016/S01683659(01)00315-7.

[336] J.W. Park, K. Hong, D.B. Kirpotin, G. Colbern, R. Shalaby, J. Baselga, Y. Shao, U.B. Nielsen, J.D. Marks, D. Moore, D. Papahadjopoulos, C.C. Benz, Anti-HER2 Immunoliposomes: enhanced efficacy attributable to targeted delivery, Clin. Cancer Res. 8 (2002) 1172-1181.

[337] T. Wickham, K. Futch, A phase I study of MM-302, a HER2-targeted liposomal doxorubicin, in patients with advanced, HER2- positive breast cancer, Cancer Res. (2012), https://doi.org/10.1158/0008-5472.sabcs12-p5-18-09 P5-18-09-P5$18-09$.

[338] J. Kattan, J.-P. Droz, P. Couvreur, J.-P. Marino, A. Boutan-Laroze, P. Rougier, P. Brault, H. Vranckx, J.-M. Grognet, X. Morge, H. Sancho-Garnier, Phase I clinical trial and pharmacokinetic evaluation of doxorubicin carried by polyisohexylcyanoacrylate nanoparticles, Investig. New Drugs 10 (1992) 191-199.

[339] Orphan Oncology Products: Produits orphelins en oncologie, http://www.onxeo. com/en/nos-produits/orphelins-oncologie/\#tabs-1-3, (2019) , Accessed date: 17 June 2019.

[340] B. Pharma, Livatag ${ }^{\circledR}$ (Doxorubicin Transdrug ${ }^{\mathrm{TM}}$ ) follow up Demonstrates significant Survival Increase in Advanced Hepatocellular Carcinoma Patients, http:// www.onxeo.com/wp-content/uploads/2014/01/110331-Livatag-EN-version.pdf, (2011) , Accessed date: 28 September 2017.

[341] J. Kopeček, H. Baẑilová, Poly[N-(2-hydroxypropyl)methacrylamide]-I. Radical polymerization and copolymerization, Eur. Polym. J. 9 (1973) 7-14, https://doi. org/10.1016/0014-3057(73)90063-3.

[342] J. Kopeček, P. Rejmanová, J. Strohalm, K. Ulbrich, B. Rihova, V. Chytry, J.B. Lloyd, R. Duncan, Synthetic polymeric drugs, US5037883A, (1991).

[343] P.A. Vasey, S.B. Kaye, R. Morrison, C. Twelves, P. Wilson, R. Duncan, A.H. Thomson, L.S. Murray, T.E. Hilditch, T. Murray, S. Burtles, D. Fraier, E. Frigerio, J. Cassidy, Phase I Clinical, Pharmacokinetic study of PK1 [N-(2Hydroxypropyl) methacrylamide Copolymer Doxorubicin]: first member of a new class of chemotherapeutic agents- drug-polymer conjugates, Clin. Cancer Res. 5 (1999) 83-94.

[344] R. Duncan, J.K. Coatsworth, S. Burtles, Preclinical toxicology of a novel polymeric antitumour agent: HPMA copolymer-doxorubicin (PK1), Hum. Exp. Toxicol. 17 (1998) 93-104, https://doi.org/10.1191/096032798678908378.

[345] J.W. Hopewel, R. Duncan, D. Wilding, K. Chakrabarti, Preclinical evaluation of the cardiotoxicity of PK2: a novel HPMA copolymer-doxorubicin-galactosamine conjugate antitumour agent, Hum. Exp. Toxicol. 20 (2001) 461-470, https://doi.org/ 10.1191/096032701682693017.

[346] V. Alakhov, E. Klinski, S. Li, G. Pietrzynski, A. Venne, E. Batrakova, T. Bronitch, A. Kabanov, Block copolymer-based formulation of doxorubicin. From cell screen to clinical trials, Colloids Surf. B: Biointerfaces 16 (1999) 113-134, https://doi. org/10.1016/S0927-7765(99)00064-8.

[347] E.V. Batrakova, A.V. Kabanov, Pluronic block copolymers: evolution of drug delivery concept from inert nanocarriers to biological response modifiers, J. Control. Release 130 (2008) 98-106, https://doi.org/10.1016/j.jconrel.2008.04.013.

[348] A. Venne, S. Li, R. Mandeville, A. Kabanov, V. Alakhov, Hypersensitizing effect of pluronic L61 on cytotoxic activity, transport, and subcellular distribution of doxorubicin in multiple drug-resistant cells, Cancer Res. 56 (1996) 3626-3629.

[349] E.V. Batrakova, T.Y. Dorodnych, E.Y. Klinskii, E.N. Kliushnenkova, O.B. Shemchukova, O.N. Goncharova, S.A. Arjakov, V.Y. Alakhov, A.V. Kabanov, Anthracycline antibiotics non-covalently incorporated into the block copolymer micelles: in vivo evaluation of anti-cancer activity, Br. J. Cancer 74 (1996) 1545-1552, https://doi.org/10.1038/bjc.1996.587.

[350] S. Danson, D. Ferry, V. Alakhov, J. Margison, D. Kerr, D. Jowle, M. Brampton, G. Halbert, M. Ranson, Phase I dose escalation and pharmacokinetic study of pluronic polymer-bound doxorubicin (SP1049C) in patients with advanced cancer, Br. J. Cancer 90 (2004) 2085-2091, https://doi.org/10.1038/sj.bjc.6601856.

[351] T. Nakanishi, S. Fukushima, K. Okamoto, M. Suzuki, Y. Matsumura, M. Yokoyama, T. Okano, Y. Sakurai, K. Kataoka, Development of the polymer micelle carrier system for doxorubicin, J. Control. Release 74 (2001) 295-302, https://doi.org/ 10.1016/S0168-3659(01)00341-8.

[352] J.A. MacDiarmid, N.B. Mugridge, J.C. Weiss, L. Phillips, A.L. Burn, R.P.P. Paulin, J.E. Haasdyk, K.A. Dickson, V.N. Brahmbhatt, S.T. Pattison, A.C. James, G. Al Bakri, R.C. Straw, B. Stillman, R.M. Graham, H. Brahmbhatt, Bacterially derived $400 \mathrm{~nm}$ particles for encapsulation and cancer cell targeting of chemotherapeutics, Cancer Cell 11 (2007) 431-445, https://doi.org/10.1016/j.ccr.2007.03.012. 
[353] B.F. El-Rayes, P.M. LoRusso, Targeting the epidermal growth factor receptor, Br. J. Cancer 91 (2004) 418-424, https://doi.org/10.1038/sj.bjc.6601921.

[354] J.A. MacDiarmid, N.B. Amaro-Mugridge, J. Madrid-Weiss, I. Sedliarou, S. Wetzel, K. Kochar, V.N. Brahmbhatt, L. Phillips, S.T. Pattison, C. Petti, B. Stillman, R.M. Graham, H. Brahmbhatt, Sequential treatment of drug-resistant tumors with targeted minicells containing siRNA or a cytotoxic drug, Nat. Biotechnol. 27 (2009) 643-651, https://doi.org/10.1038/nbt.1547.

[355] B.J. Solomon, J. Desai, M. Rosenthal, G.A. McArthur, S.T. Pattison, S.L. Pattison, J. MacDiarmid, H. Brahmbhatt, A.M. Scott, A first-time-in-human phase I clinical trial of bispecific antibody-targeted, paclitaxel-packaged bacterial minicells, PLoS
ONE 10 (2015) e0144559, , https://doi.org/10.1371/journal.pone.0144559.

[356] J. Mendelsohn, J. Baselga, Epidermal growth factor receptor targeting in cancer, Semin. Oncol. 33 (2006) 369-385, https://doi.org/10.1053/j.seminoncol.2006. 04.003.

[357] I.K. Mellinghoff, M.Y. Wang, I. Vivanco, D.A. Haas-Kogan, S. Zhu, E.Q. Dia, K.V. Lu, K. Yoshimoto, J.H.Y. Huang, D.J. Chute, B.L. Riggs, S. Horvath, L.M. Liau, W.K. Cavenee, P. Nagesh Rao, R. Beroukhim, T.C. Peck, J.C. Lee, W.R. Sellers, D. Stokoe, M. Prados, T.F. Cloughesy, C.L. Sawyers, P.S. Mischel, Molecular determinants of the response of glioblastomas to EGFR kinase inhibitors, N. Engl. J. Med. 353 (2005) 2012-2024, https://doi.org/10.1056/NEJMoa051918. 